\title{
Parametrical Investigation of Piezoelectric Energy Harvesting via Friction-Induced Vibration
}

\author{
D. W. Wang $\mathbb{D}^{1}{ }^{1}$ M. X. Liu, ${ }^{1}$ W. J. Qian, ${ }^{2}$ X. Wu, ${ }^{1}$ Q. Ma, ${ }^{1}$ and Z. Q. Wu ${ }^{1}$ \\ ${ }^{1}$ Science and Technology on Reactor System Design Technology Laboratory, Nuclear Power Institute of China, \\ Chengdu 610213, China \\ ${ }^{2}$ School of Mechatronic Engineering, Southwest Petroleum University, Chengdu 610500, China \\ Correspondence should be addressed to D. W. Wang; 1776135059@qq.com
}

Received 15 September 2019; Revised 12 January 2020; Accepted 21 January 2020; Published 19 February 2020

Academic Editor: Marcello Vanali

Copyright $(2020$ D. W. Wang et al. This is an open access article distributed under the Creative Commons Attribution License, which permits unrestricted use, distribution, and reproduction in any medium, provided the original work is properly cited.

In this work, piezoelectric energy harvesting performance via friction-induced vibration is investigated numerically. A onedegree-of-freedom friction system with a piezoelectric element is proposed, to study the piezoelectric energy harvesting via friction-induced stick-slip vibration. Subsequently, a two-degree-of-freedom friction system with two piezoelectric elements is proposed, to investigate the piezoelectric energy harvesting via model coupling vibration. Results show that regardless of the friction systems, it is feasible to convert friction-induced vibration energy to electrical energy when the friction system is operating in the unstable vibration region. Parametrical analysis indicates that for the one-degree-of-freedom friction system, when the normal load increases from $5 \mathrm{~N}$ to $30 \mathrm{~N}$, the stick-slip motion becomes more intense, and the friction system will generate more electric energy. While for the two-degree-of-freedom friction system, with the normal load increase from $20 \mathrm{~N}$ to $120 \mathrm{~N}$, there is a critical normal load value for the generation of the strongest vibration and the highest voltage output. When the velocity of the belt increases from $0.5 \mathrm{~m} / \mathrm{s}$ to $2 \mathrm{~m} / \mathrm{s}$, the amplitudes of vibration and output voltage become larger. While with the velocity further increasing, the stick-slip motion and generated electric energy disappear. For both friction systems, the external electric resistance has no effect on the dynamic behaviour of the friction system; however, it can modify the output voltage amplitudes within limits. It is also found that when the force factor of piezoelectric element increases from $3.1 \times 10^{-5} \mathrm{~N} / \mathrm{V}$ to $3.1 \times 10^{-3} \mathrm{~N} / \mathrm{V}$, the vibration and harvested energy gradually increase. When the force factor further increases to $3.1 \times 10^{-2} \mathrm{~N} / \mathrm{V}$, the vibration reduces drastically and the corresponding output voltages reduce significantly, which proves that a piezoelectric element with an appropriated force factor can give the highest harvested energy and conversion efficiency.

\section{Introduction}

With rapid advances of wireless technologies, a large number of new low-power electronic devices emerged in the last decades, which have been widely used in our daily life [1]. The basic requirement for these devices is that they should be able to operate for long enough periods of time solely on battery power, considering that the replacement or recharging of the battery sometimes is of high cost and is impossible [2-4]. Therefore, a great amount of research has been carried out to develop energy harvesting technology to supply power for these electronic devices.

During the last decades, vibration energy harvesting technology has attracted much attention since a vibration source can be easily obtained from an ambient environment [5-10]. Many comprehensive reviews or published books on vibration energy harvesting have been presented [7-10]. Generally, there are four approaches to convert vibration to electric energy, i.e., electromagnetic, electrostatic, piezoelectric, and triboelectric [11]. Among them, piezoelectric energy harvesting is extensively studied because of its high power density and nonreliance on an external magnetic field or initial voltage [12-15]. Moreover, piezoelectric materials can be utilized flexibly since it can be directly attached to the mechanical system to convert mechanical vibration energy into electrical energy via the deformation of piezoelectric material (such as attached in the cantilever beam). 
Although ambient vibration energy harvesting technology has been studied for many years, the greatest challenge of this technology is the energy output and conversion efficiency of vibration energy harvesters at a particular ambient excited frequency. If the frequency of ambient vibration deviates slightly from the resonant frequency of the energy harvester, the resulting energy output and conversion efficiency of harvester would be reduced drastically [10]. To overcome this problem, many new technologies have been proposed, such as structure modification [16-22], circuit design [23-27], nonlinear techniques [28-33], and so on. Although these approaches can improve the energy harvesting performance to some extent, they inevitably increase the complexity and uncertainty of the energy harvesters. In addition, the potential ambient vibration energy sources exhibit a random form and exist in a wide range of frequencies; thus, these uncertainty factors also bring great challenges for the design of energy harvesters.

Accordingly, a possible approach to overcome this problem is proposed: i.e., converting another type of vibration energy which does not rely on ambient vibration source, such as self-excited vibration. This type of vibration does not require any exterior force excitation, but it can take place under specific working conditions which result in the destabilization of the system itself [34]. There are two typical kinds of self-excited vibration, i.e., flow-induced vibration and friction-induced vibration [34]. The energy harvesting via flow-induced vibration has seen a large increase in applications, and the energy harvesters using an aeroelastic instability mechanism called the galloping have been examined [35]. Abdelkefi [36] reviewed recent studies in the field of energy harvesting from aeroelastic energy harvesting. However, the relevant studies on the energy harvesting by exploiting a friction-induced vibration phenomenon are rarely reported. As a matter of fact, the friction-induced vibration phenomenon can be seen in many mechanical applications which contain sliding frictional contact, such as brake systems, mechanical gear systems, wiper blade systems, lead screw drives, and engines and power trains [37-39]. The friction-induced vibration generated from the friction systems is often regarded as harmful to them; thus, the research emphasis of friction-induced vibration is focused on its generation mechanism [40-44] and suppression approaches [45-49]. However, sometimes, the friction-induced vibration can be useful, for example, in musical instruments and even in energy harvesting. For a real brake system, the friction-induced vibration can be generated during brake process, which often accompanies with unwanted noise. If piezoelectric elements are introduced into the brake system, strains may be generated in the piezoelectric elements due to the vibration caused by friction, which can consequently convert the strain energy into electrical energy. Similarly, for other mechanical systems, such as conveyer system, rail-wheel system, and gear system, friction-induced vibration energy can be considered as a source for energy harvesting.

As described in some of the recent review papers, the most possible mechanisms for the generation of friction-induced vibration is stick-slip motion and model coupling phenomenon [50]. Helseth [51] proposed a piezoelectric energy harvester using stick-slip motion between hooks and loops, and they found that the harvester would collect maximum power at a certain normal load and sliding velocity. Tadokoro et al. [52] experimentally and numerically studied the stickslip motion between a steel ball and a steel plate in the lubricated condition and verified the feasibility of energy harvesting through stick-slip motion. Masuda and Sawai [53] performed a preliminary study to detect the stick-slip energy harvesting by using an L-shaped cantilever sliding on a friction pad surface, and they obtained the value of harvested energy and the corresponding conversion efficiency. Heffel and Hagedorn [54] also experimentally and numerically studied a wobbling disc energy harvester and found that converting friction-induced vibration to electrical energy could lead to a broadband applicability, since the operation frequency of the piezoelectric element was independent of the excitation signal. These studies are suitable for realizing a new energy harvesting approach by using friction-induced vibration. However, the knowledge on the energy harvesting via friction-induced vibration is still limited, and a systematic study about the effect of working parameters on energy harvesting remains unknown and somehow mysterious. Therefore, in order to get a better understanding of the basic principle of energy harvesting via friction-induced vibration, more fundamental research should be carried out. This paper makes such a contribution.

In this work, the piezoelectric energy harvesting by exploiting friction-induced vibration is systemically studied numerically. A one-degree-of-freedom friction system (mass sliding on belt) with a piezoelectric element attached is proposed; two kinds of friction laws are, respectively, introduced into the system to investigate the piezoelectric energy harvesting behaviour of the friction system related to stick-slip motion. Subsequently, a two-degree-of-freedom friction system with piezoelectric elements which, respectively, ignores and considers the separation between the mass and belt is analyzed, to detect the effect of mode coupling motion on the piezoelectric energy harvesting. After analysing the different friction systems, some conclusions on the effect of working parameters (load, velocity, external electric resistance, and force factor of piezoelectric element) of the friction systems on the piezoelectric energy harvesting are presented.

\section{Numerical Analysis of the One-Degree-of- Freedom Friction System}

2.1. One-Degree-of-Freedom Friction System. A one-degreeof-freedom friction system is created in this study, as shown in Figure 1. This system contains a point mass $m$ and a moving rigid belt. A normal load $F_{N}$ is imposed onto $m$ to create friction contact between $m$ and the belt. The belt is moving at a constant velocity $v_{0}$. A spring $k$ and damper $c$ are connected with $m$ in the horizontal direction. In addition, a piezoelectric element called Piezo is introduced into the horizontal direction of the friction system, which is connected with external electric resistance $R$. In this 


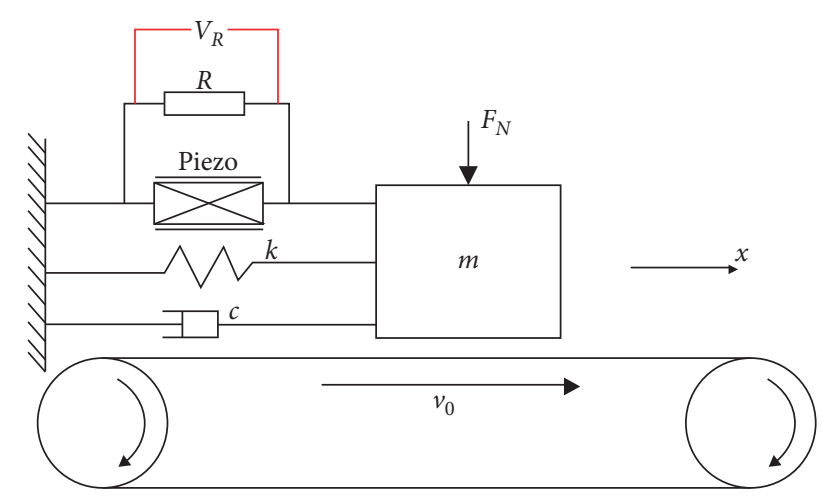

FIGURE 1: One-degree-of-freedom friction system with piezoelectric element.

system, it is assumed that a light and thin piezoelectric film (polyvinylidene fluoride) is attached with the mass, the spring $k$ contains the stiffness of piezoelements, and the mass of the piezoelements is included in the $m$. Thus, the piezoelectric element can convert horizontal mechanical vibration energy into electrical energy when friction-induced vibration generates during the friction process. This friction system is similar to the model proposed by Tadokoro et al. [52]. Friction force $F_{r}$ is determined by relative velocity $v_{r}$ between $m$ and the belt, which is defined as $v_{r}=v_{0}-\dot{x} . V_{R}$ is the voltage across external electric resistance $\mathrm{R}$.

Thus, the mechanical and piezoelectric coupling equation of this one-degree-of-freedom friction system can be written as

$$
\left\{\begin{array}{l}
m \ddot{x}(t)+c \dot{x}(t)+k x(t)+\alpha V_{R}(t)=F_{r}, \\
\alpha \dot{x}(t)=\frac{C_{p} \dot{V}_{R}(t)+V_{R}(t)}{R},
\end{array}\right.
$$

where $\alpha$ is a force factor of the piezoelectric element and $C_{p}$ is its clamped capacitance. Based on the value of relative sliding velocity $v_{r}$, the friction system has two motion phases: slip phase $\left(v_{r} \neq 0\right)$ and stick phase $\left(v_{r}=0\right)$. Therefore, the friction system is a typical nonsmooth system. For the convenience of numerical computation, the criterion conditions for the relative velocity $v_{r} \neq 0$ and $v_{r}=0$ are changed to $\left|v_{r}\right|>\varepsilon$ and $\left|v_{r}\right| \leq \varepsilon$, respectively, where $\varepsilon$ represents a threshold value for the sliding velocity which is sufficiently close to zero.

Therefore, when the $\left|v_{r}\right|>\varepsilon$, i.e., the friction system is in the slip phase, the friction force $F_{r}$ is expressed as

$$
F_{r}=\mu_{k} F_{N} \operatorname{sgn}\left(v_{r}\right) .
$$

On the other hand, when the $\left|v_{r}\right| \leq \varepsilon$, i.e., the friction system is in the stick phase, the friction force $F_{r}$ is determined by the maximum static friction force $F_{\text {static }}$ and the applied force $F_{\text {applied }}$ on the mass. Here, the $F_{\text {static }}$ is expressed as

$$
F_{\text {static }}=\mu_{s} F_{N} \operatorname{sgn}\left(v_{r}\right),
$$

and the applied force is expressed as

$$
F_{\text {applied }}=k x(t)+c \dot{x}(t)+\alpha V_{R}(t) .
$$

Therefore, the friction force $F_{r}$ of the system in the stick phase can be expressed as

$$
F_{r}= \begin{cases}k x(t)+c \dot{x}(t)+\alpha V_{R}(t), & \text { when the }\left|F_{\text {applied }}\right|<\mu_{s} F_{n}, \\ \mu_{s} F_{n} \operatorname{sgn}\left(v_{\text {rel }}\right), & \text { when the }\left|F_{\text {applied }}\right| \geq \mu_{s} F_{n} .\end{cases}
$$

Then, equation (1) can be transformed to the following form:

$$
\left\{\begin{array}{l}
\ddot{x}(t)=\frac{F_{r}}{m}-\frac{c}{m} \dot{x}(t)-\frac{k}{m} x(t)-\frac{\alpha}{m} V_{R}(t), \\
\dot{V}_{R}(t)=\frac{\alpha}{C_{p}} \dot{x}(t)-\frac{V_{R}(t)}{R C_{p}} .
\end{array}\right.
$$

Subsequently, the fourth-order Runge-Kutta method is used to solve the differential equations.

In the following numerical investigations, it is assumed that $m=1 \mathrm{~kg}, k=1 \mathrm{~N} / \mathrm{m}$, and $c=0.1 \mathrm{~N} /(\mathrm{m} / \mathrm{s})$. Assuming the clamped capacitance of the piezoelectric element $C_{p}=7.2 \times 10^{-6} \mathrm{~F}$. The parameters of piezomaterial are referred to the work done by Tadokoro et al. [52]. Considering that for the discrete system, the stick-slip motion is caused by the velocity-weakening friction, while the wave propagation at the interface will cause stick-slip motion in the continuous system; thus, two typical friction laws in which the coefficient of friction versus relative velocity $\left(v_{r}\right)$ are illustrated in Figure 2, which is denoted as I and II in the following studies. For the friction law I, it is a well-known Coulomb-Amontons friction characteristic, in which the static friction coefficient $\mu_{s}=0.4$ and the kinetic friction coefficient $\mu_{k}$ is kept equal to 0.15 during sliding process. However, for the friction law II, the kinetic friction coefficient $\mu_{k}$ continuously varies with the relative velocity. Regardless of the friction laws, the friction force $F_{r}$ is determined by the friction phases, i.e., stick phase, slip phase, or transition phase. In the following sections, the dynamic behaviour and energy harvesting performance of the friction system for these two different friction laws are analyzed.

\subsection{Parametric Study}

2.2.1. Effect of Normal Load on the Dynamics and Energy Harvesting. Figure 3 shows the dynamical performance and output voltage of the friction system with the variation of constant normal load in friction law I. In this analysis, assuming the force factor $\alpha=3.1 \times 10^{-5} \mathrm{~N} / \mathrm{V}$, external electric resistance $R$ is fixed at $20000 \Omega$ and belt velocity $v_{0}$ is fixed at $1 \mathrm{~m} / \mathrm{s}$. Both of the initial displacement and velocity of the mass are set zero in the stick-slip simulation analysis. At a lower normal load $(5 \mathrm{~N})$, no stick-slip motion can be observed during the sliding process and the vibration and output voltage signals gradually disappear. With the increase of the normal load $(10 \mathrm{~N})$, saw tooth wave-like motion appears in the displacement curve, suggesting the 


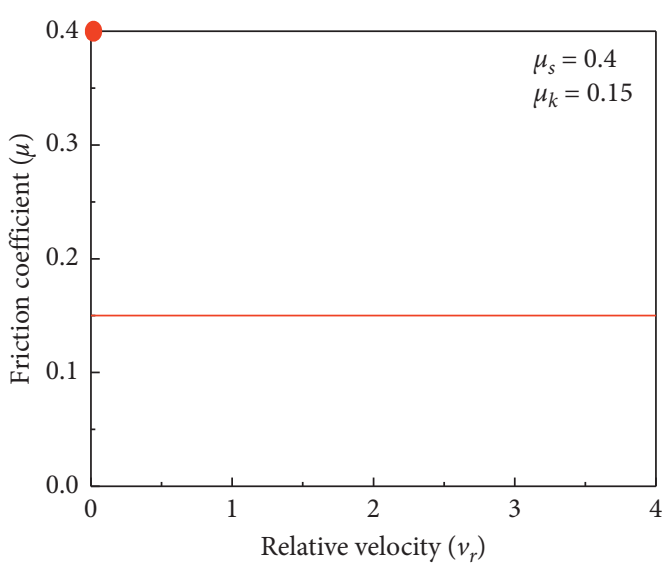

(a)

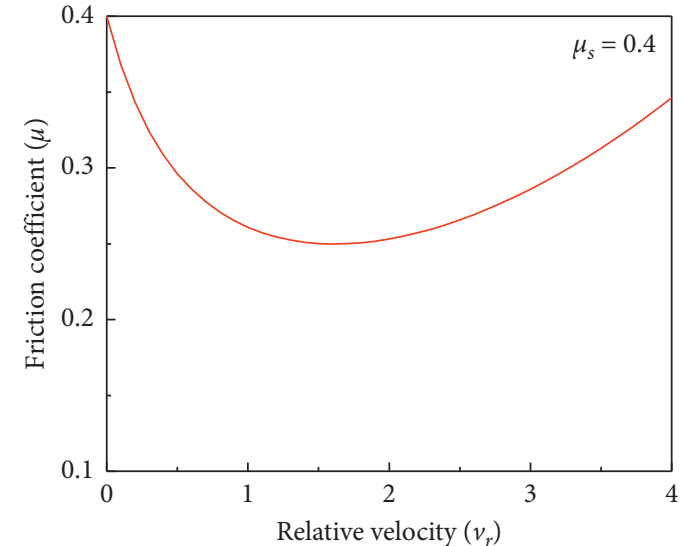

(b)

FIgURe 2: Friction law I: $\mu_{s}=0.4$ and $\mu_{k}=0.15(\mathrm{a})$ and friction law II: $\mu_{k}=0.25 /\left(1+1.42\left|v_{r}\right|\right)+0.15+0.01\left(v_{r}\right)^{2}(\mathrm{~b})$.

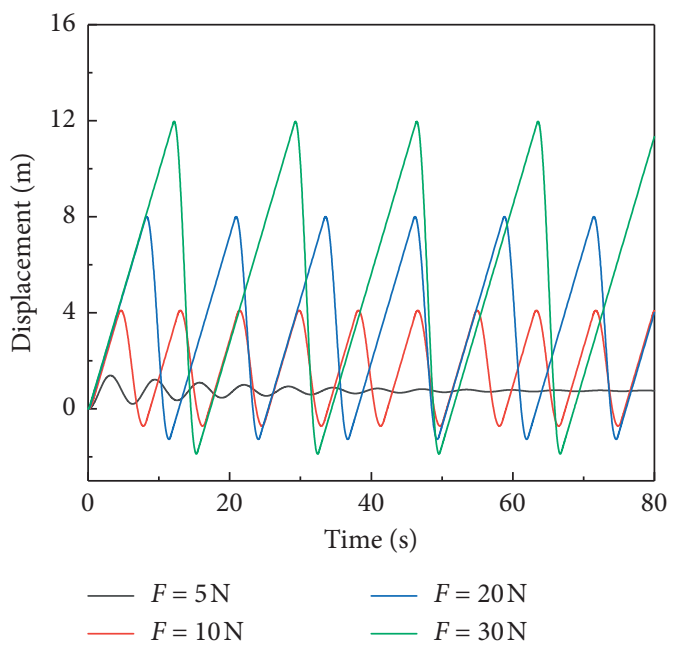

(a)

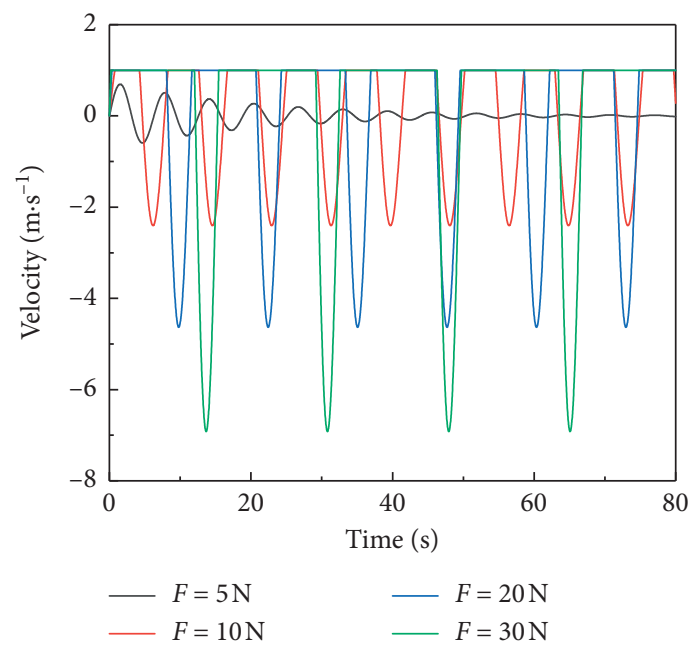

(b)

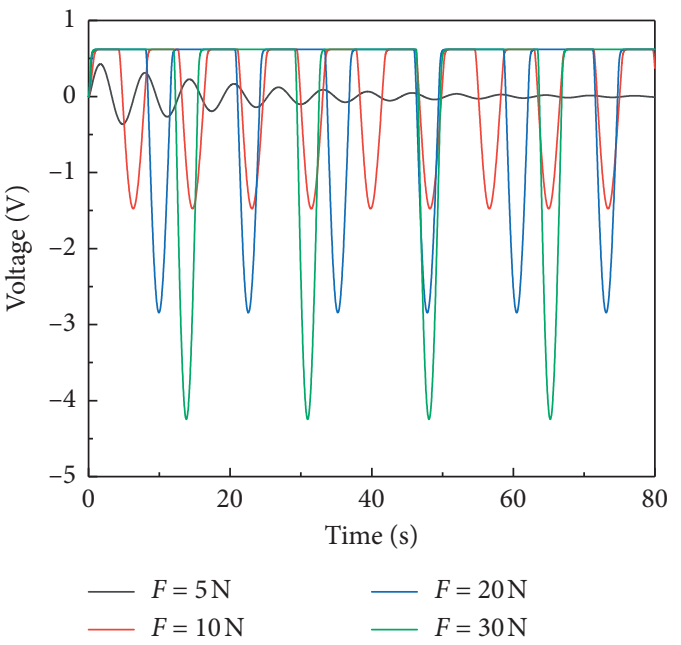

(c)

Figure 3: Vibration displacement (a), velocity (b), and output voltage (c) of the friction system with different normal loads in the friction law I. 
occurrence of stick-slip motion. During the periods when the displacement curve shows a positive slope, the velocity of $m$ is consistent with belt velocity $v_{0}$, which means that the friction system stays in the stick phase. When the velocity of $m$ is different from $v_{0}$, the friction system is in the slip phase. In addition, a sustained voltage output can be observed when the system generates stick-slip motion, and the waveform of the output voltage is similar to that of the velocity. This phenomenon indicates the feasibility of energy harvesting via stick-slip vibration. Since the friction force increases with the increase in normal load, the friction system needs to store more energy to achieve the transition from stick to slip. Visibly, stronger stick-slip motion with higher amplitude of vibration and output voltage can be observed, while the frequency of the stick-slip motion gradually reduces.

Similar phenomenon can be observed in the case of friction law II, as shown in Figure 4. Visible voltage output can be found when the stick-slip motion is generated from the friction system, and the waveform of the output voltage is similar to that of the velocity. The stick-slip frequency becomes smaller with the increase in normal load, whilst the magnitude of vibration and output voltage gradually increase with the increase in normal load. Furthermore, Table 1 summarizes the RMS values of the output voltage of the friction system with the variation of normal load. It can be seen that regardless of the friction laws, larger normal load is beneficial for generating higher voltage, which consequently suggests that larger load can generate more electrical energy.

The total harvested energy value and the energy conversion efficiency corresponding to different normal loads are calculated according to the following expression:

$$
P_{E}=\int_{0}^{T}\left(\frac{V_{R}^{2}}{R}\right) \mathrm{d} t
$$

and the energy conversion efficiency is written as

$$
\zeta=\left(\frac{P_{E}}{P_{F}}\right) \times 100,
$$

where $P_{E}$ represents the electric energy, $V_{R}$ represents the output voltage, and $R$ is the external electric resistance. $P_{F}$ represents the work caused by friction, which can be expressed as [52]

$$
P_{F}=\int_{0}^{T} F_{r} v_{0} \mathrm{~d} t
$$

where $F_{r}$ and $v_{0}$ represent the friction force and the driving speed, respectively. Figure 5(a) shows the harvested energy value and the energy conversion efficiency of the friction system with the variation of normal load in the friction law I. The red dashed line indicates the moment that stick-slip solutions begin to dominate the response. Larger harvested energy can be observed at a larger normal load, and the reason is attributed to the larger input energy into the friction system. In addition, a visible shift from low to high in the conversion efficiency can be observed as long as the friction system generates stick-slip motion, which further indicates that the energy can be harvested via friction-induced vibration. The conversion efficiency value will not show visible change during the stick-slip motion period, as can be seen in Figure 5(a) that the conversion efficiency value slightly fluctuated near the value of $0.002 \%$. The convention efficiency of this model is relatively low; this is due to the fact that during friction process, a large amount of friction energy is dissipated in the form of heat and only a small part is dissipated in the form of vibration. In this work, only the vibration energy is converted to the electric energy, and the convention efficiency is calculated by using the electric energy divided by the friction energy. Therefore, the convention efficiency is relatively low.

In the case of friction law II, the harvested energy value and the energy conversion efficiency with the variation of normal load is shown in Figure 5(b). It can be seen that the variation of total harvested energy has the similar tendency as the case of friction law I, which gradually increases with the increase of normal load, and the conversion efficiency also shows a visible shift from low to high value once the system exhibits stick-slip solutions. However, unlike the previous case that the conversion efficiency value stays in a relative stable value with further increase of normal load, a critical load for the highest conversion efficiency can be observed in the case of friction law II.

\subsubsection{Effect of Velocity on the Dynamics and Energy} Harvesting. Figures 6 and 7 show the displacement, velocity, and output voltage of the friction system with different belt velocities in the friction laws I and II, respectively. Assume the force factor $\alpha$ is equal to $3.1 \times 10^{-5} \mathrm{~N} / \mathrm{V}$. The normal load in this analysis is kept at $20 \mathrm{~N}$, and load resistance $R$ is fixed at $20000 \Omega$. Similar dynamics and voltage variation trend can be observed for both friction laws I and II. The slope of the displacement curve in the stick section becomes steeper and stick-slip frequency becomes larger with the sliding velocity becoming larger. In addition, the output voltage exhibits larger amplitude at higher velocity. While with the velocity further increasing, the stick-slip motion of the friction system disappears; as a consequence, the output voltage shows a considerable decrease.

The reason for the abovementioned physical phenomenon is that when the sliding speed is small, the velocity of the mass can easily reach the belt velocity during the vibration process and form the stick and slip motions. When the belt velocity increases gradually, the difference between the mass vibration velocity and the belt velocity increases as well and the mass and belt are always in the situation of relative motion; thus, the stick-slip motion gradually disappears with the increase of belt velocity and the output voltage disappears accordingly.

The harvested energy value and the energy conversion efficiency of the friction system at different velocities are shown in Figure 8. For both friction laws, with the increase of sliding velocity, the friction system is able to harvest more vibration energy in the stick-slip regions and the conversion efficiency also exhibits a relative high value in the stick-slip region. But with further increase of the velocity, the stick-slip motion disappears and the harvested energy shows drastic reduction. It is worth noting that for the case of friction law I, 


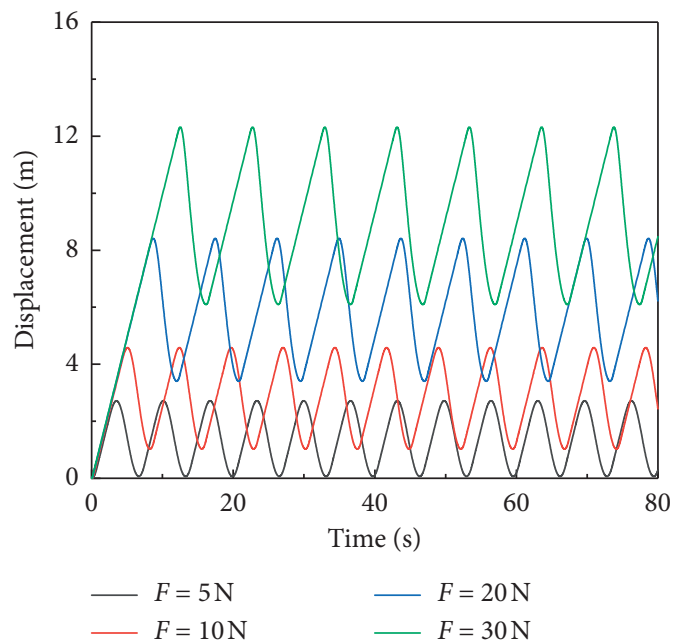

(a)

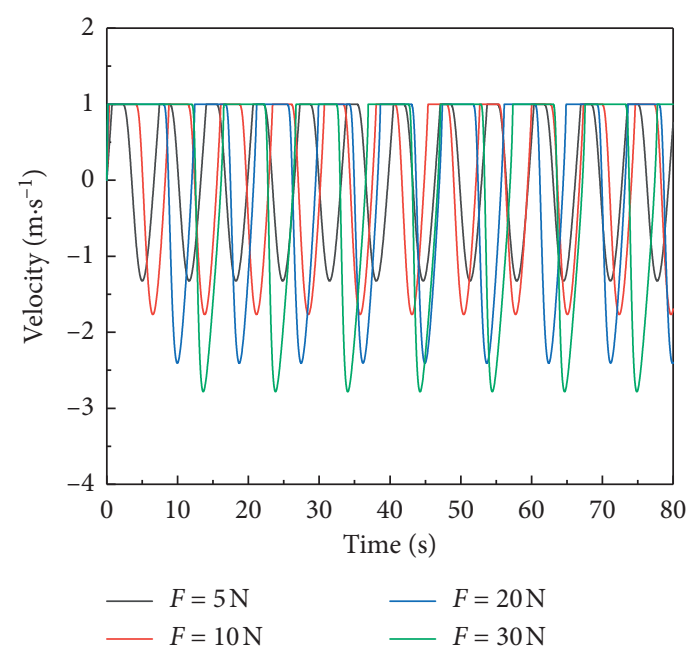

(b)

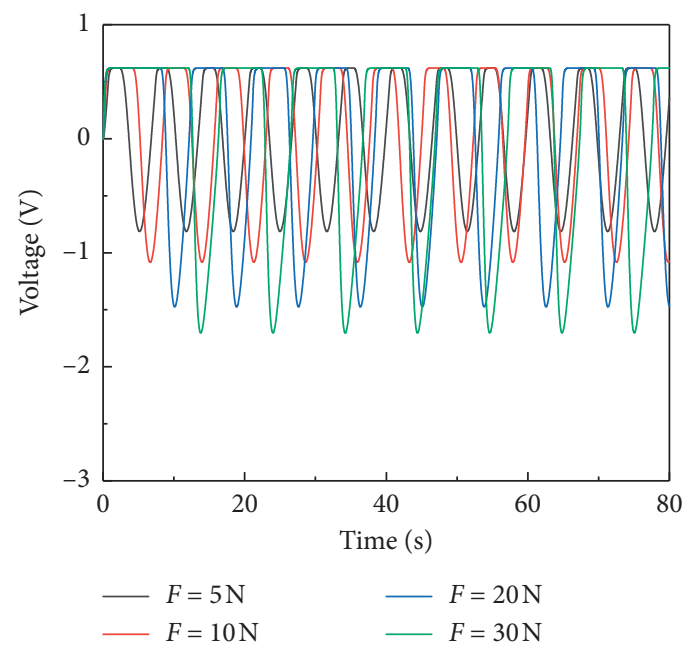

(c)

Figure 4: Vibration displacement (a), velocity (b), and output voltage (c) of the friction system with different normal loads in the friction law II.

TABLE 1: The RMS of output voltage of the friction system with the variation of normal load.

\begin{tabular}{lccccc}
\hline & Force & $5 \mathrm{~N}$ & $10 \mathrm{~N}$ & $20 \mathrm{~N}$ & $30 \mathrm{~N}$ \\
Friction law I & Voltage RMS & $0.132 \mathrm{~V}$ & $0.67 \mathrm{~V}$ & $1.03 \mathrm{~V}$ & $1.41 \mathrm{~V}$ \\
Friction law II & Voltage RMS & $0.52 \mathrm{~V}$ & $0.59 \mathrm{~V}$ & $0.71 \mathrm{~V}$ & $0.79 \mathrm{~V}$ \\
\hline
\end{tabular}

the conversion efficiency stays at a relative stable value when the system exhibits stick-slip motion, in contrast with the case of friction coefficient law II, which leads to a critical velocity for the highest conversion efficiency. Similar variation tendency of dynamics and energy harvesting performance related to the sliding velocity can be found in [52]; however, it is not shown that the larger velocity will cause the disappearance of the stick-slip and considerable reduction of the energy conversion efficiency.

2.2.3. Effect of External Electric Resistance on the Dynamics and Energy Harvesting. The effect of external electric resistance on the displacement, velocity, and output voltage of the friction system is studied, as shown in Figures 9 and 10. The normal load in this analysis is kept at $20 \mathrm{~N}$, belt velocity is fixed at $1 \mathrm{~m} / \mathrm{s}$, and force factor $\alpha$ is equal to $3.1 \times 10^{-5} \mathrm{~N} / \mathrm{V}$. It is worth noting that the purpose of this section is not finding an optimal external resistance for the friction system to produce the most electric energy. It is a single-variable analysis to detect the effect of different resistances on the energy harvesting behaviour of the friction system. Therefore, the values of load and velocity are arbitrarily chosen and fixed.

For both friction laws, it is found that the variation of external electric resistance seems to not affect dynamical behaviour of the friction system considerably, since the displacement and velocity signals show no modification at different external electric resistances (this will be discussed in the following section). However, the external electric resistance can significantly affect the amplitude of output voltage to a certain degree. The output voltage shows a 


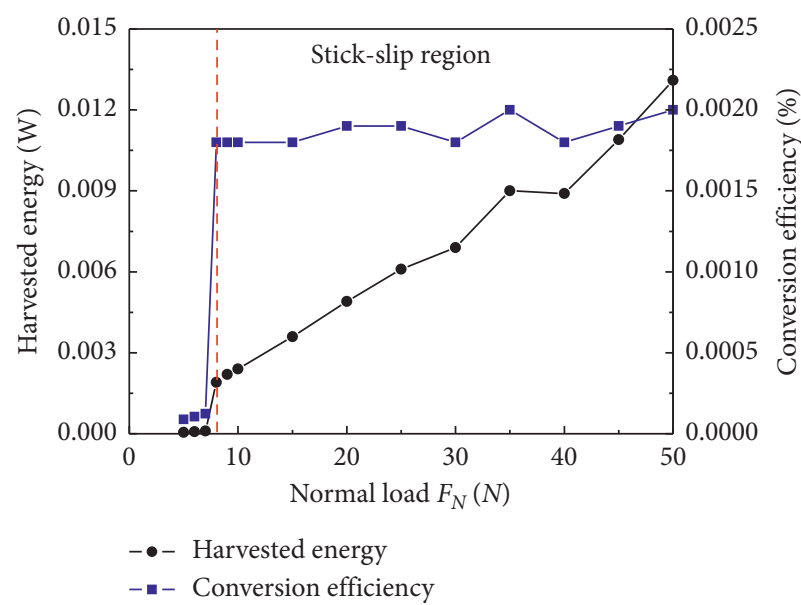

(a)

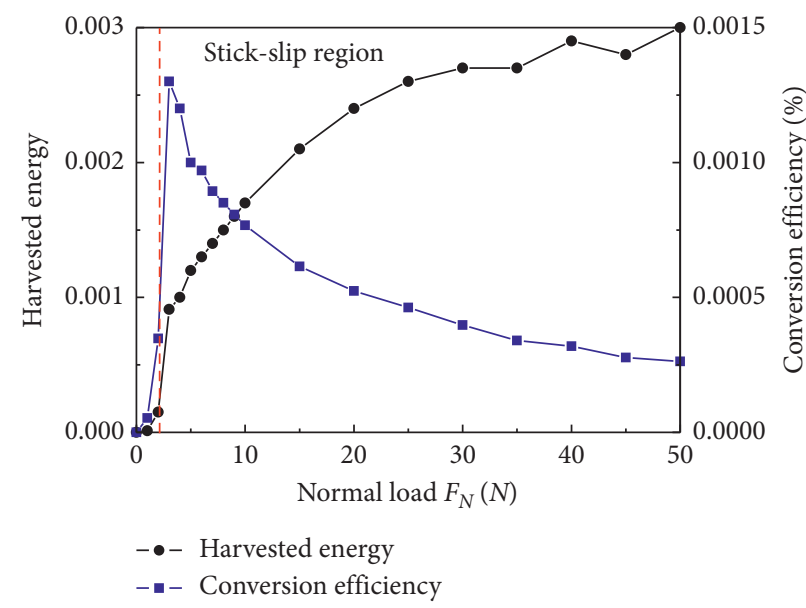

(b)

FIgURE 5: Harvested energy and conversion efficiency of the friction system with different normal loads in the friction laws I (a) and II (b).

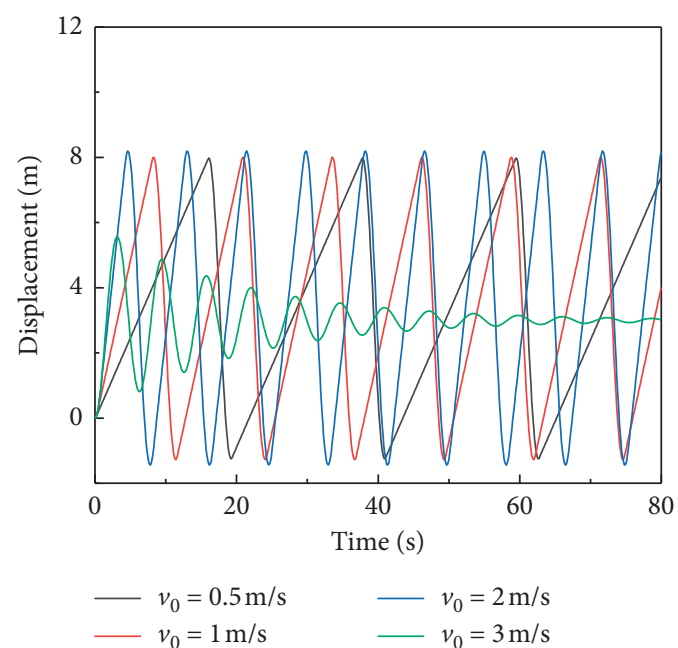

(a)

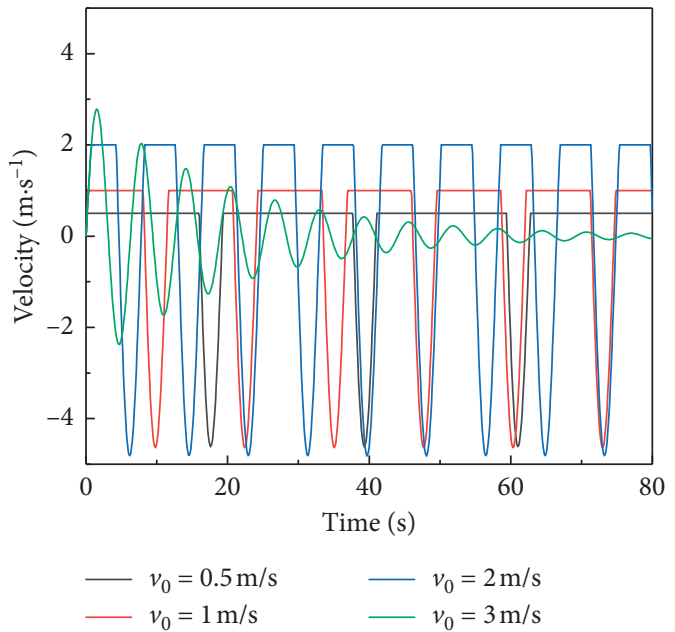

(b)

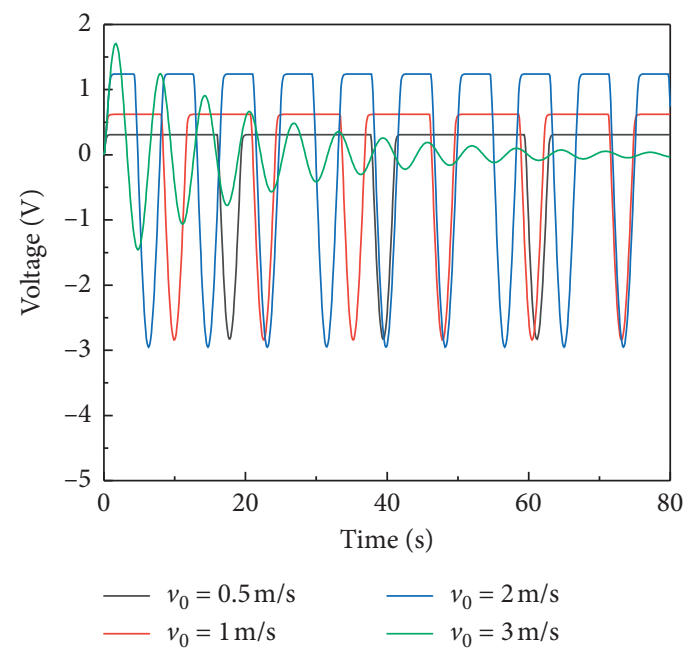

(c)

FIgURE 6: The displacement (a), velocity (b), and output voltage (c) of the friction system with different velocities in the friction law I. 


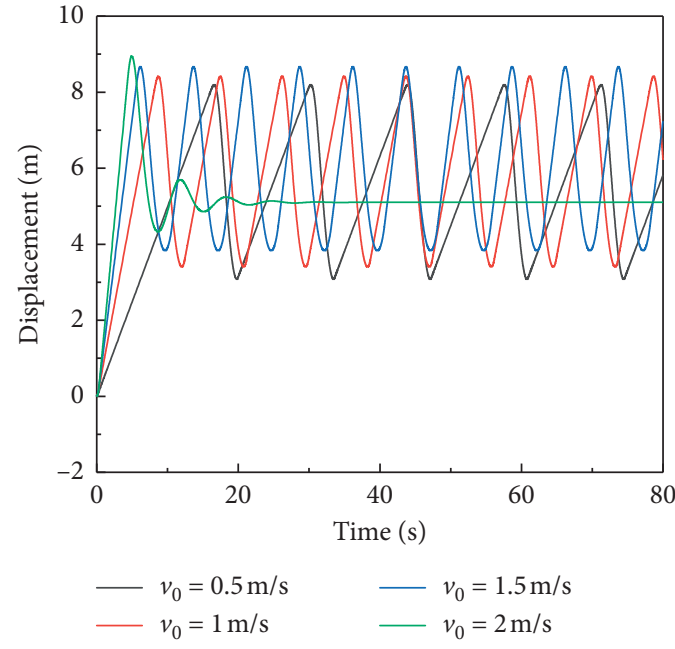

(a)

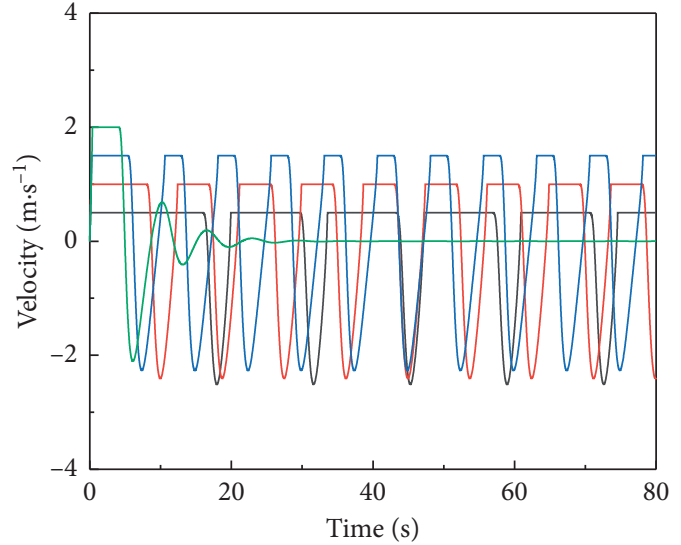

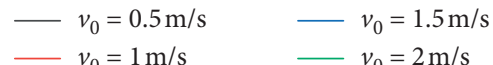

(b)

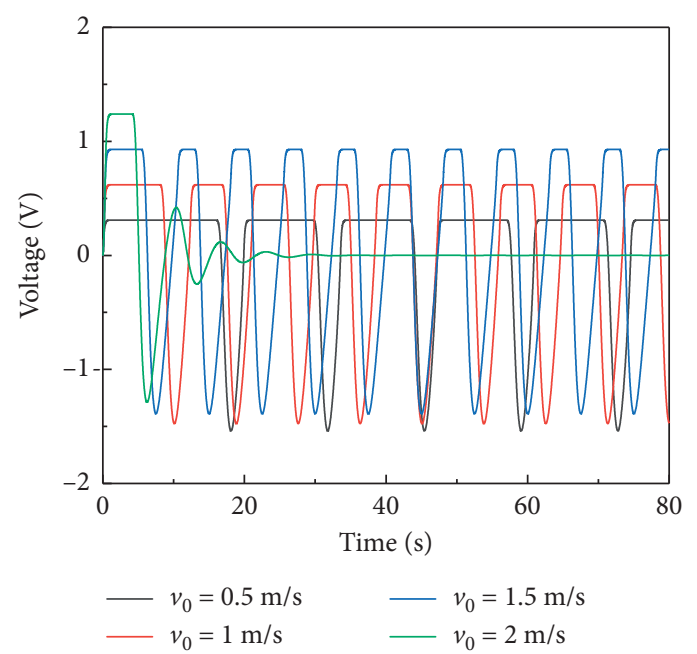

(c)

FIGURE 7: The displacement (a), velocity (b), and output voltage (c) of the friction system with different velocities in the friction law II.

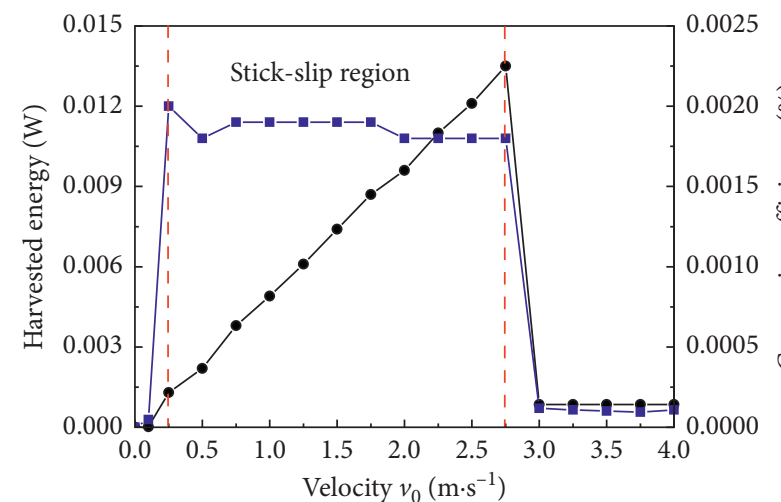

- - Harvested energy

- - Conversion efficiency

(a)

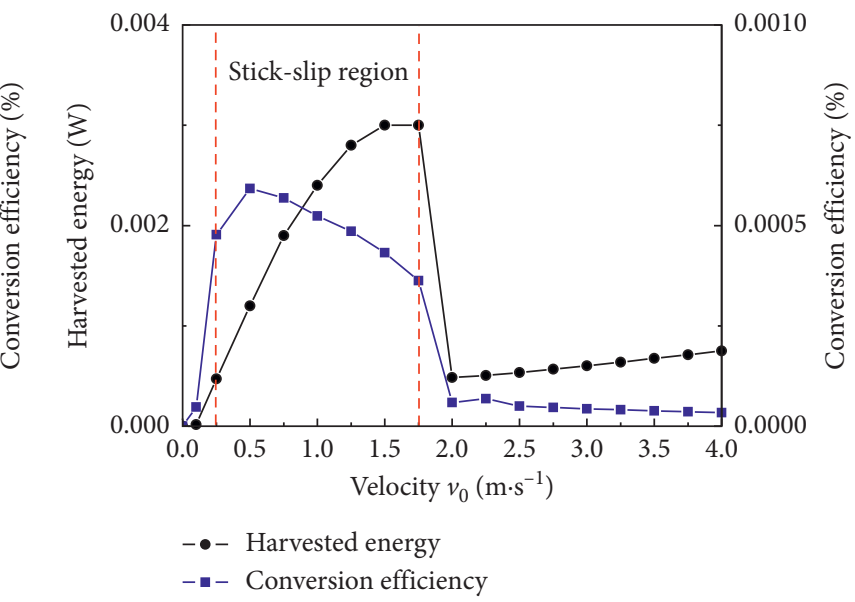

(b)

FIGURE 8: The harvested energy and conversion efficiency of the friction system with different sliding velocities in the friction laws I (a) and II (b). 

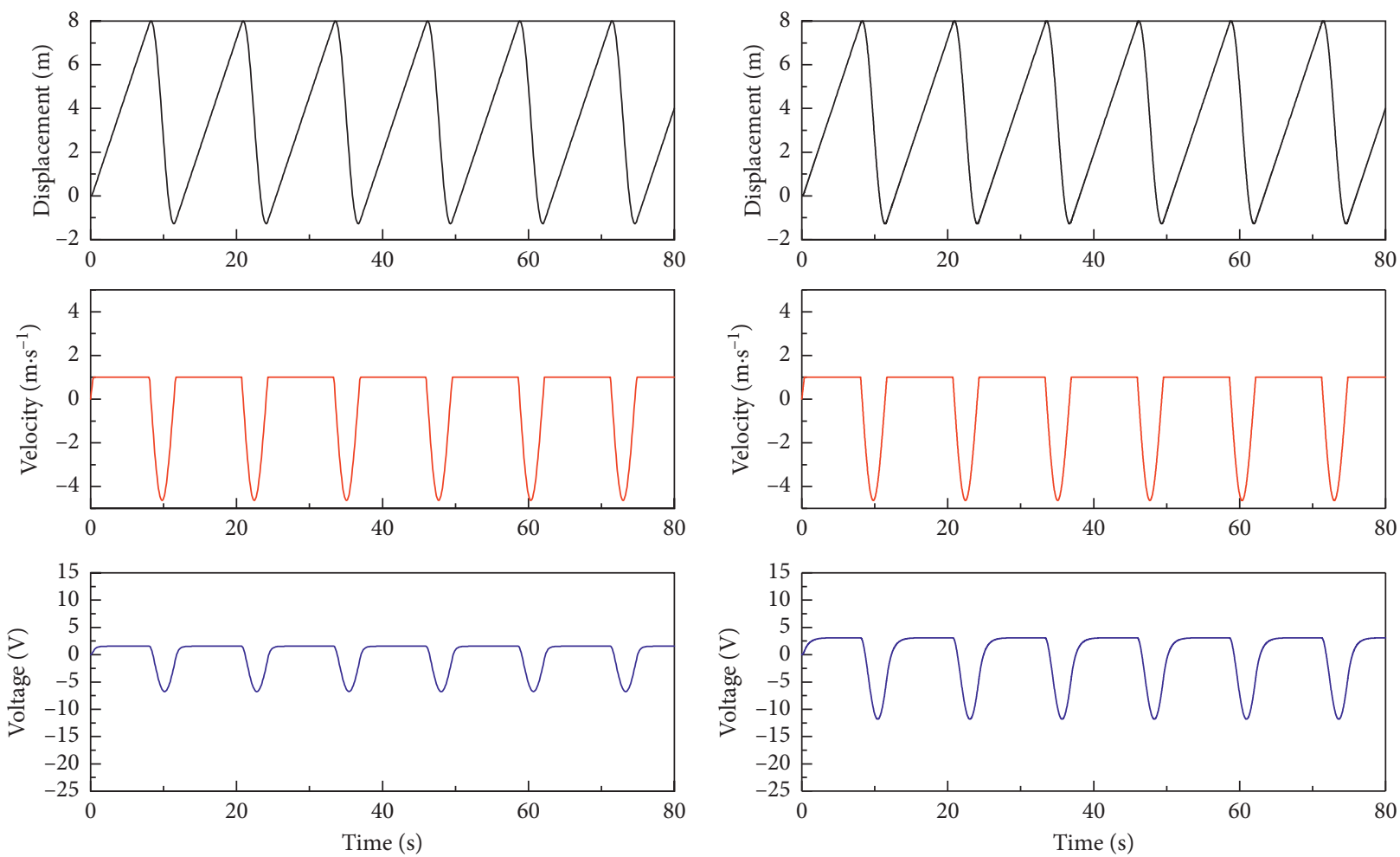

(a)

(b)
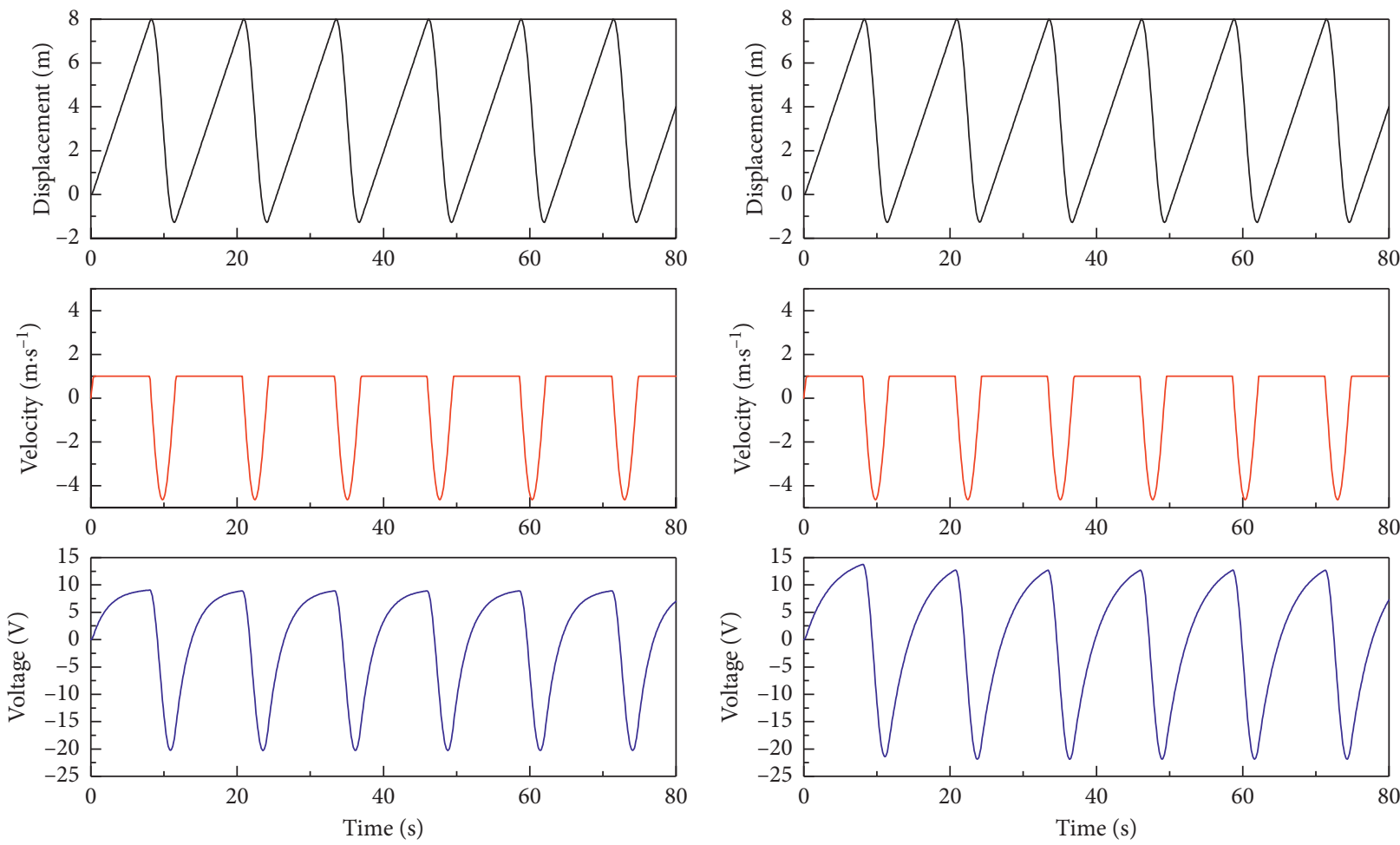

(c)

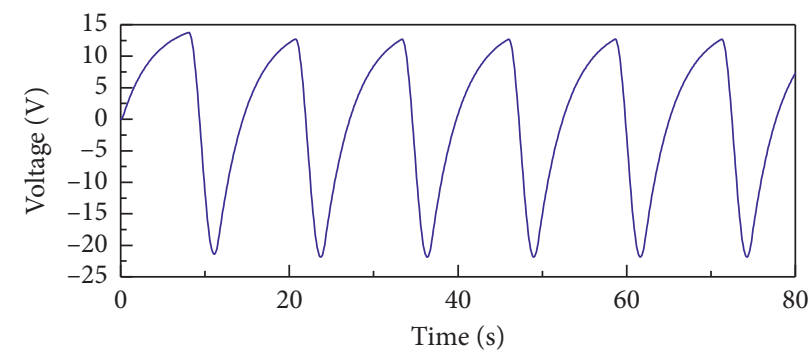

(d)

FIgURE 9: The displacement, velocity, and output voltage of the friction system when the load resistance is $R=50000 \Omega$ (a), $R=100000 \Omega$ (b), $R=300000 \Omega$ (c), and $R=500000 \Omega$ (d) in the friction law I. 

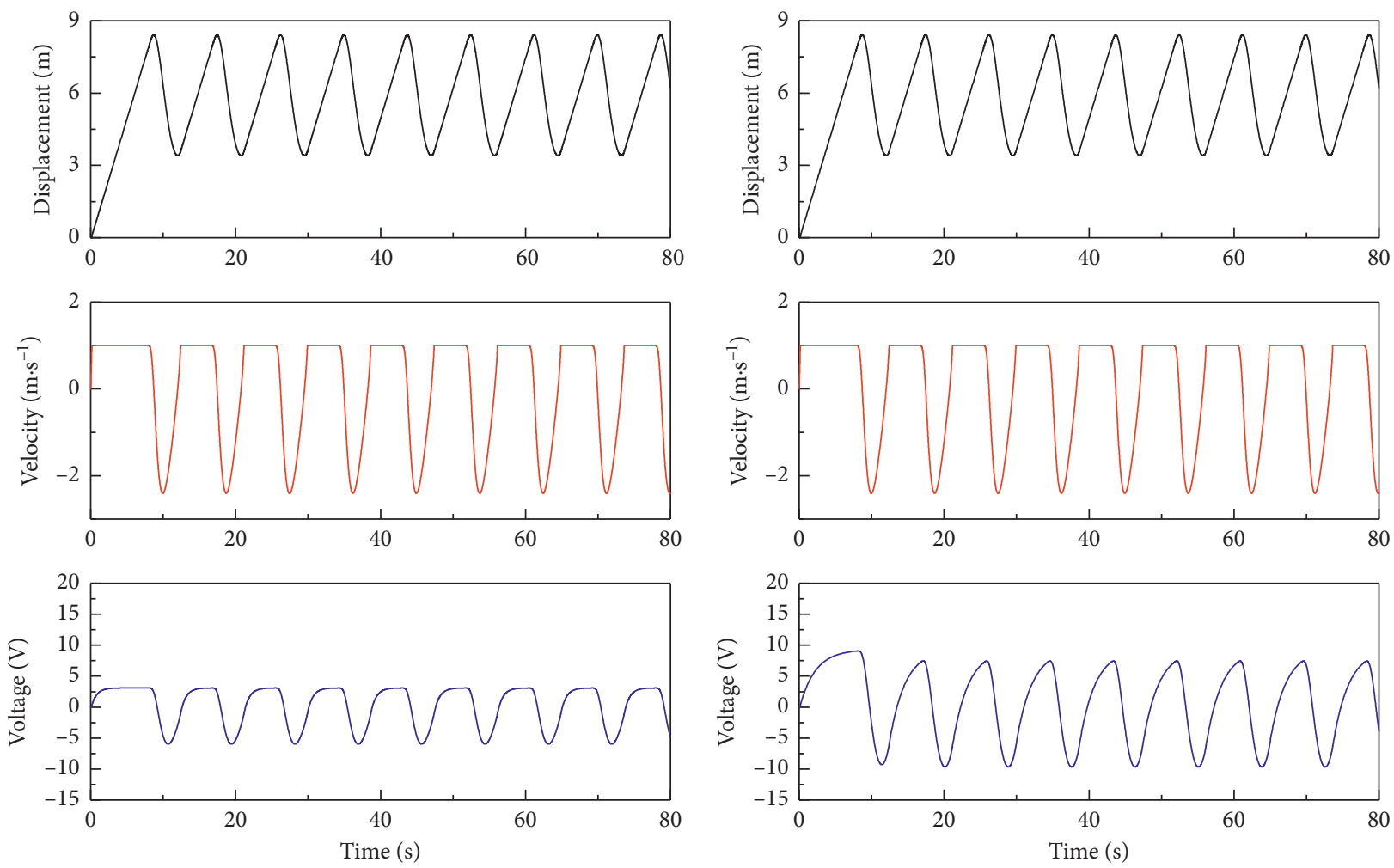

(a)

(b)
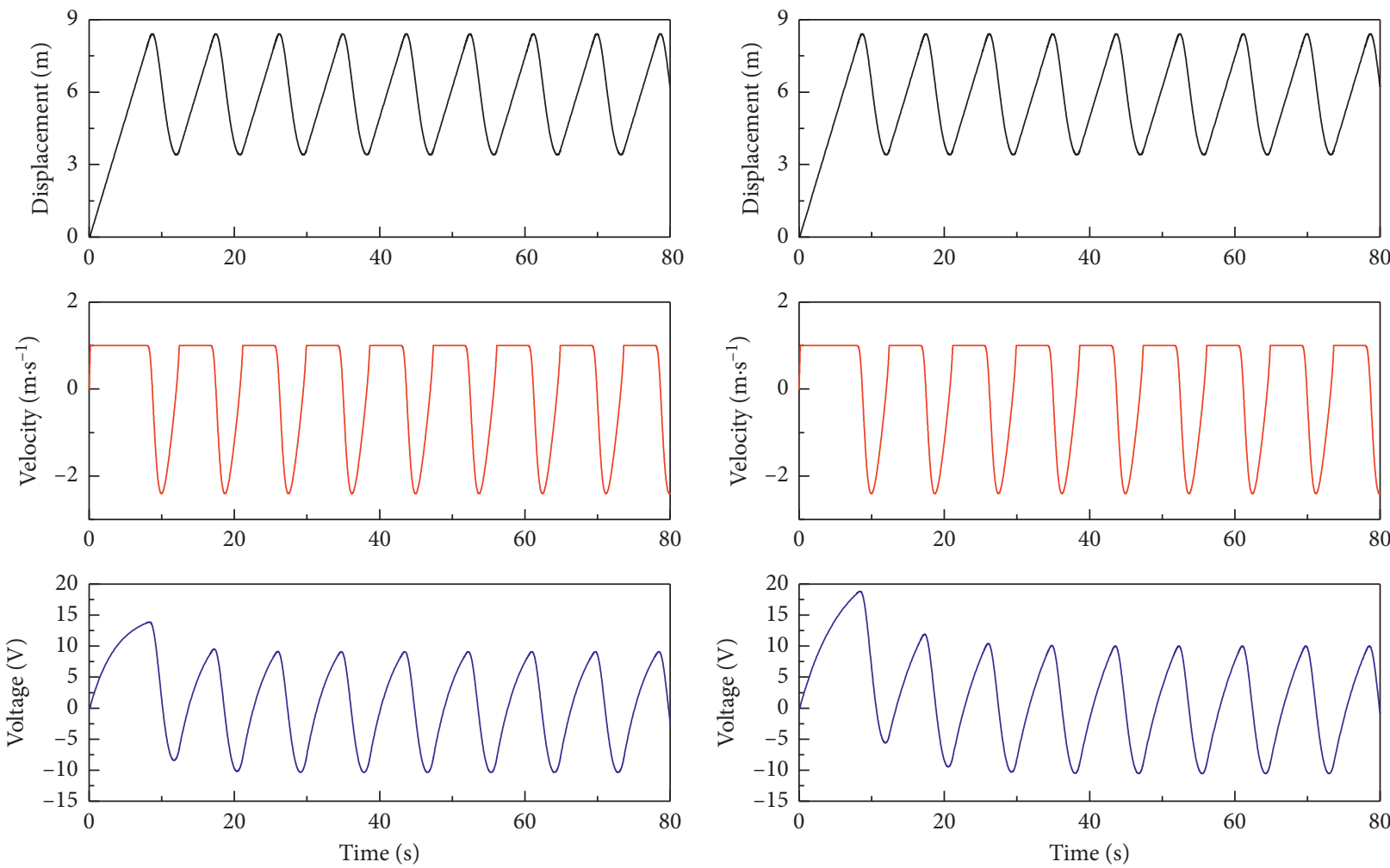

(c)

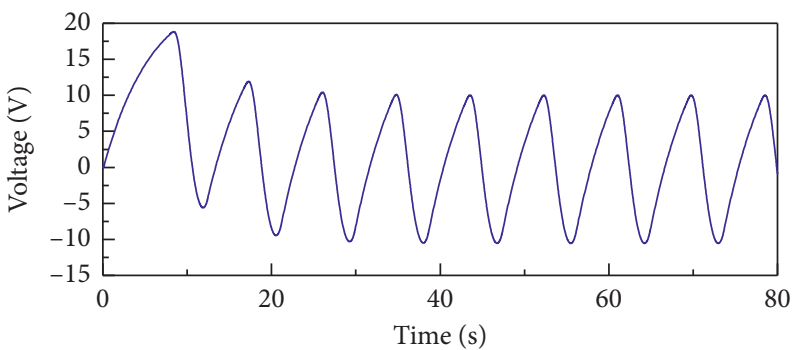

(d)

Figure 10: The displacement, velocity, and output voltage of the friction system when the load resistance is $R=100000 \Omega$ (a), $R=300000 \Omega$ (b), $R=500000 \Omega$ (c), and $R=800000 \Omega$ (d) in the friction law II. 
visible increase with the increase of load resistance in a certain range. However, with the load resistance further increasing, the output voltage will not exhibit a visible change and the amplitude of output voltage gradually tends to be steady.

The harvested energy value and the energy conversion efficiency at different external electric resistances of the friction system are calculated, as shown in Figure 11. For both of the two friction laws, both the harvested energy and conversion efficiency exhibit a trend of rising first and then decrease with the continuous increase of electric resistances, suggesting that there is a critical electric resistance value for the occurrence of highest harvested energy and conversion efficiency. This is because that when the external electric resistance is over a certain value, although the amplitude of output voltage still shows slight increase with the further increase of the load resistance, the amplitude of the voltage increase is less than that of the load resistance increment. Thus, according to equations (7) and (8), the total harvested energy and conversion efficiency start to decrease with the further increase of the external electric resistances. The inherent reason for this phenomenon is the match between the source impedance and the load impedance; the harvested energy and conversion efficiency can be optimized based on a specific load resistance, and the magnitude is related to electrical impedance matching of the piezoelectric material insert and the interface circuit. This phenomenon is in accordance with the experimental results found by Masuda and Sawai [53], who observed that there was a critical external electric resistance to give the best energy harvesting performance during stick-slip motion.

In this work, it is found that the resistive load does not affect the dynamic response of the system, while it has a noticeable effect on the output voltage. On the other hand, according to equation (1), the dynamics and voltages are coupling with each other in this equation. They are paradox with each other. Therefore, it is worth analysing the form of equation (1) and explaining the reason of this paradox phenomenon. From the first equation of equation (1), the $\alpha V_{R}(t)$ is introduced into the motion equation of the friction system. It is worth noting that the force factor $\alpha$ used in this work $\left(3.1 \times 10^{-5} \mathrm{~N} / \mathrm{V}\right)$ is very small; thus, the effect of $\alpha V_{R}(t)$ on the first equation of equation (1) is very slight, which causes the very weak coupling between the dynamics and voltage. Therefore, the displacement and velocity signals can be directly calculated through the equation of $m \ddot{x}(t)+c \dot{x}(t)+k x(t)=F_{r}$, and $R$ has no effect on the displacement and velocity signals. Actually, it is found that the vibration signals calculated from $\alpha=3.1 \times 10^{-5} \mathrm{~N} / \mathrm{V}$ is the same as those results from $\alpha=0 \mathrm{~N} / \mathrm{V}$.

To further verify that the paradox phenomenon is caused by the small $\alpha$, a relative larger $\alpha\left(3.1 \times 10^{-3} \mathrm{~N} / \mathrm{V}\right)$ is set in the simulation process; the displacement, velocity, and output voltage of the friction system are calculated in the friction law I (i.e., $\mu_{s}=0.4$ and $\left.\mu_{k}=0.15\right)$. As the results shown in Figure 12, the increase of $R$ causes the reduction of the vibration amplitude, increase of the vibration frequency, and increase of output voltage as well. While with the further increase of $R$, since that the item of $V_{R}(t) / R$ (second equation of equation (1)) is too small to affect the dynamic behaviour, the dynamics and voltage signals do not show significant variation. Therefore, the effect of external electric resistance on the dynamics is dependent on the value of force factor $\alpha$. For a small $\alpha$, since the $\alpha V_{R}(t)$ is too small to affect the motion of system, the dynamics are not relied on $R$. While for a large $\alpha$, the increase of $R$ will reduce the vibration amplitude, increase the vibration frequency, and increase the output voltage to a certain degree. In addition, with the further increase of $R$, since the item of $V_{R}(t) / R$ is too small to affect the dynamic behaviour, the dynamics do not show significant variation with the further increase of $R$.

\subsubsection{Effect of Force Factor on the Dynamics and Energy} Harvesting. The force factor of a piezoelectric element reflects how large a force is required to produce a voltage of $1 \mathrm{~V}$. Considering that the force factor links the motions and piezoelectricity of the friction system, it is worth exploring the relations between the force factor and dynamics and energy harvesting, which will be beneficial for the choice of similar piezoelectricity material with different force factors to maximize harvested energy via friction-induced vibration. It worth noting that in this work, the piezoelement used in the numerical analysis is not a stack; instead, it is regarded as a light and thin piezoelectric film; thus, it is assumed that the cross section, the thickness, and other parameters of the piezoelectric thin film are fixed values as well and only the force factor is varied in this section [52]. Figures 13 and 14 plot the variation of dynamic behaviour and output voltages of the friction system with different force factors in friction laws I and II, respectively. The normal load in this analysis is kept at $20 \mathrm{~N}$, the belt velocity is fixed at $1 \mathrm{~m} / \mathrm{s}$, and the external electric resistance $R$ is fixed at $20000 \Omega$. It is seen that the amplitudes of displacement and velocity show a reduction tendency with the force factor increasing from $3.1 \times 10^{-5} \mathrm{~N} / \mathrm{V}$ to $5 \times 10^{-3} \mathrm{~N} / \mathrm{V}$. However, the output voltage values show considerable increases at a larger factor. While with the force factor further increasing, the stick-slip motion of the friction system disappears; as a consequence, the output voltage shows a considerable decrease. It is worth noting that in Figures 13(d) and 14(d), the velocity signals show oscillation at the initial period, while there is no oscillation shown in the displacement signals. This is because that the velocity signals still oscillate along the positive direction, which indicates that the mass still slides along the positive direction, and thus, the displacement signal does not exhibit oscillation; instead, the displacement shows a tendency of gradual increase.

Figure 15 illustrates the harvested energy and the conversion efficiency of the friction system with the variation of force factor. The abscissa is expressed as the logarithmic form of the force factor. For the two friction laws, both the harvested energy and conversion efficiency show a tendency 

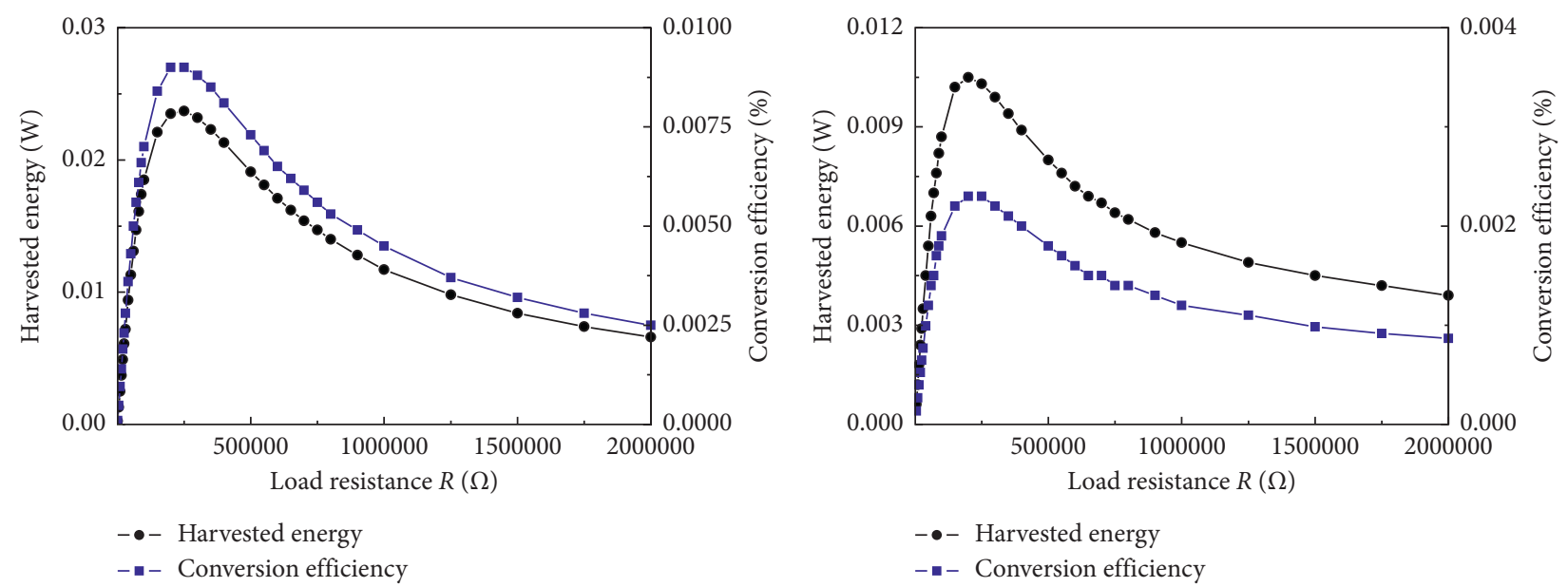

(a)

(b)

FIGURE 11: Harvested energy and conversion efficiency of the friction system with different external electric load resistances in the friction laws I (a) and II (b).
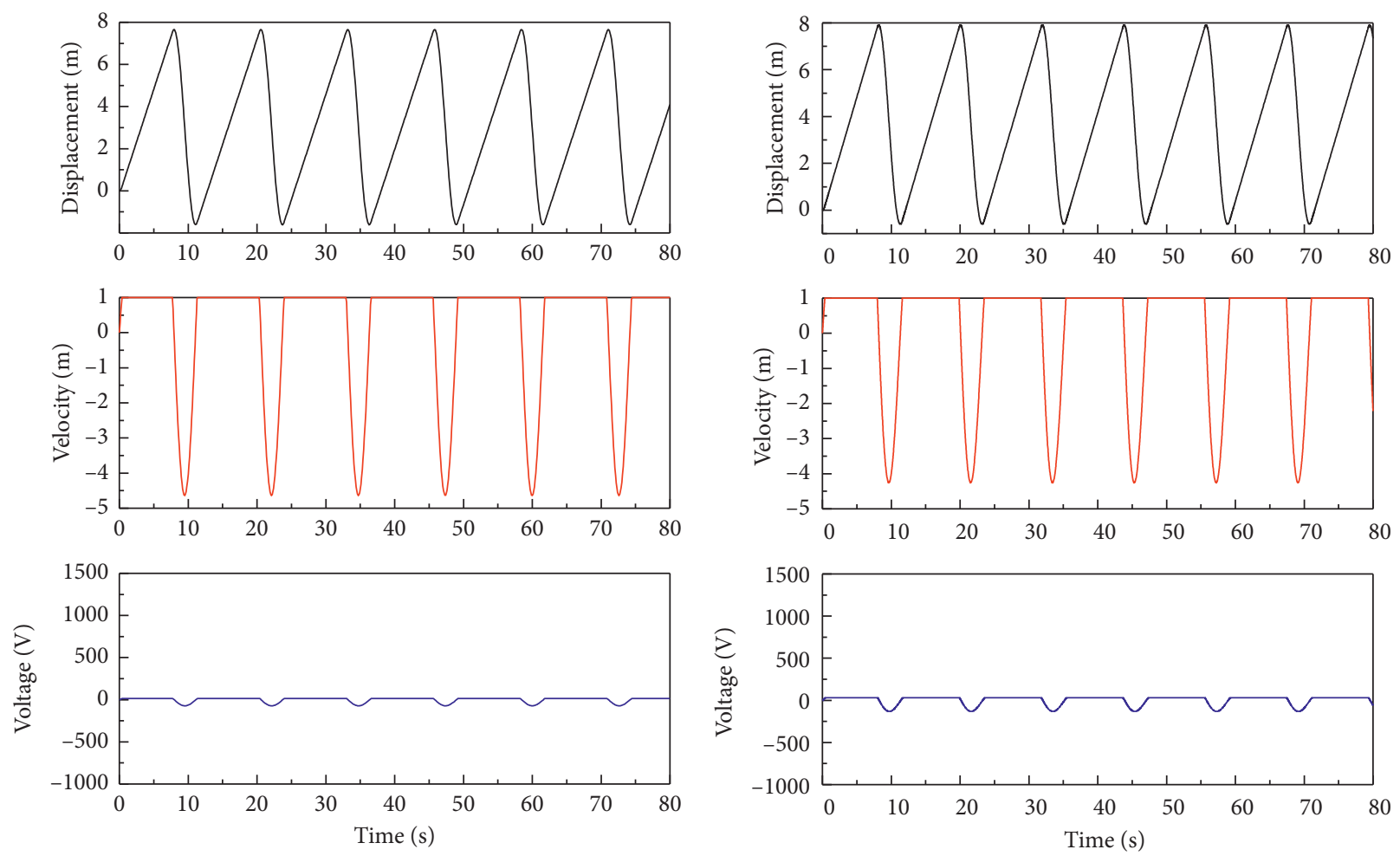

(a)

(b)

Figure 12: Continued. 

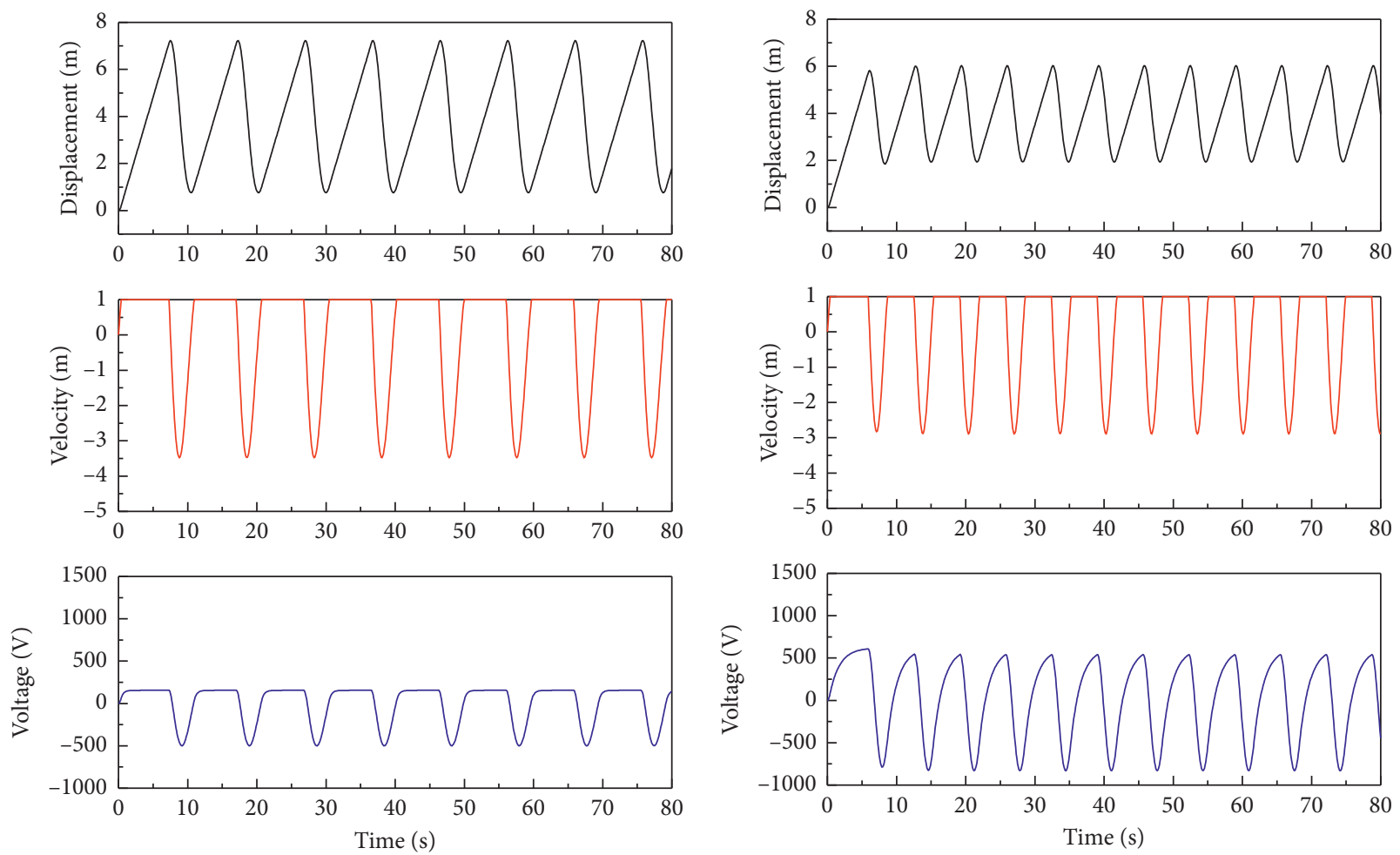

(c)

(d)
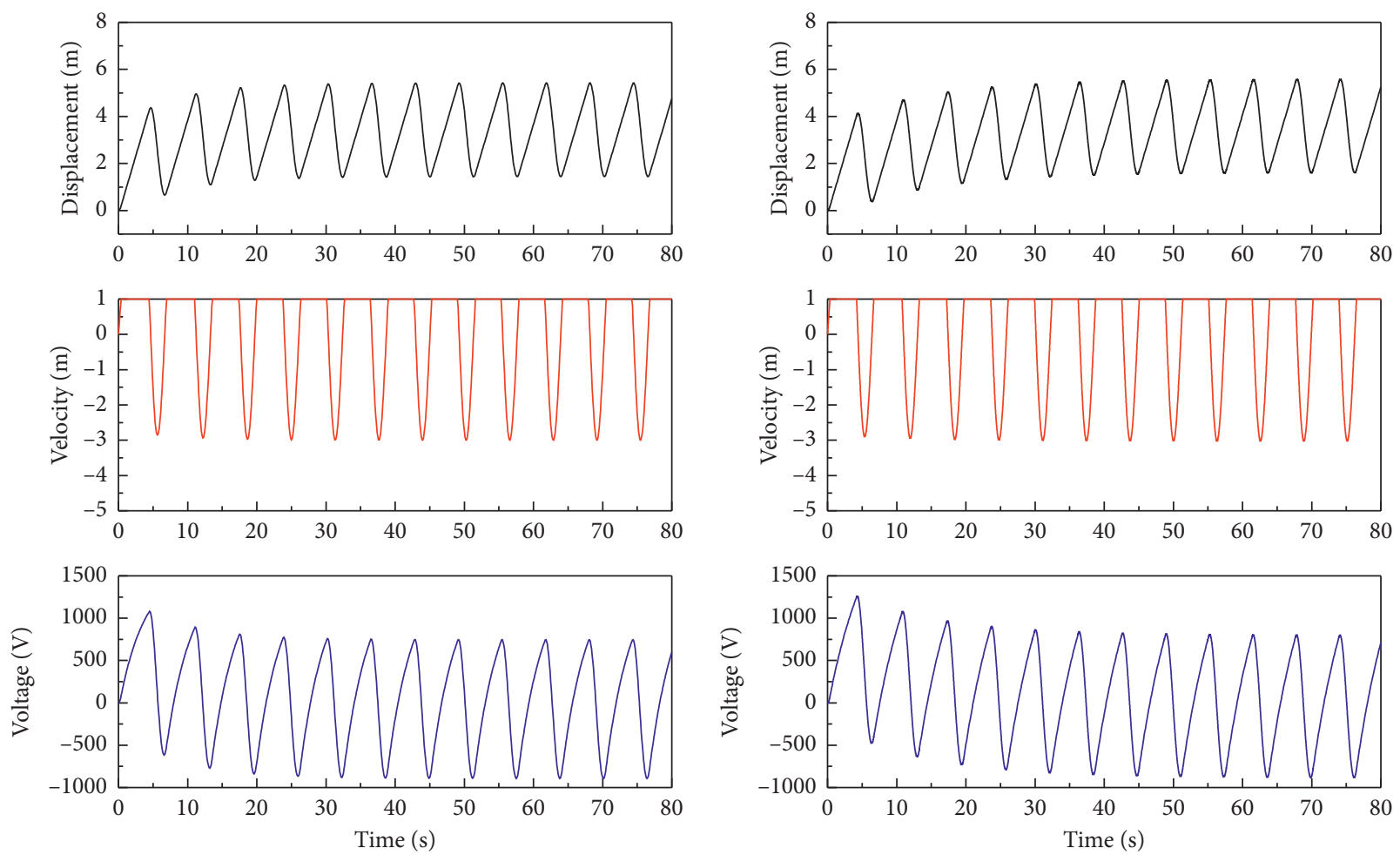

(e)

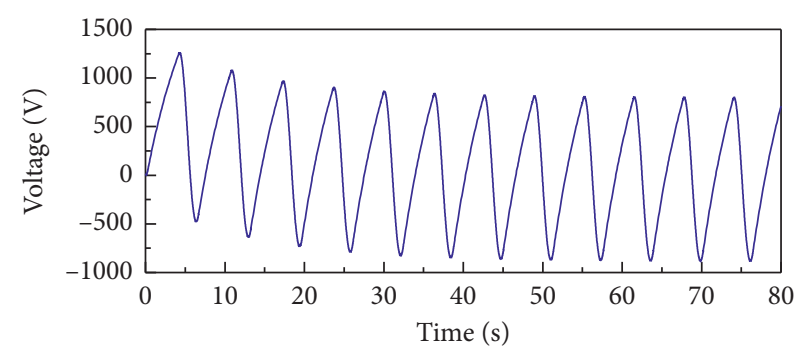

(f)

FIGURE 12: The dynamics and voltage signals of the friction system with different external electric resistance $R$ in a large $\alpha$. (friction law I): (a) $R=50000 \Omega$, (b) $R=10000 \Omega$, (c) $R=50000 \Omega$, (d) $R=200000 \Omega$, (e) $500000 \Omega$, and (f) $R=800000 \Omega$. 

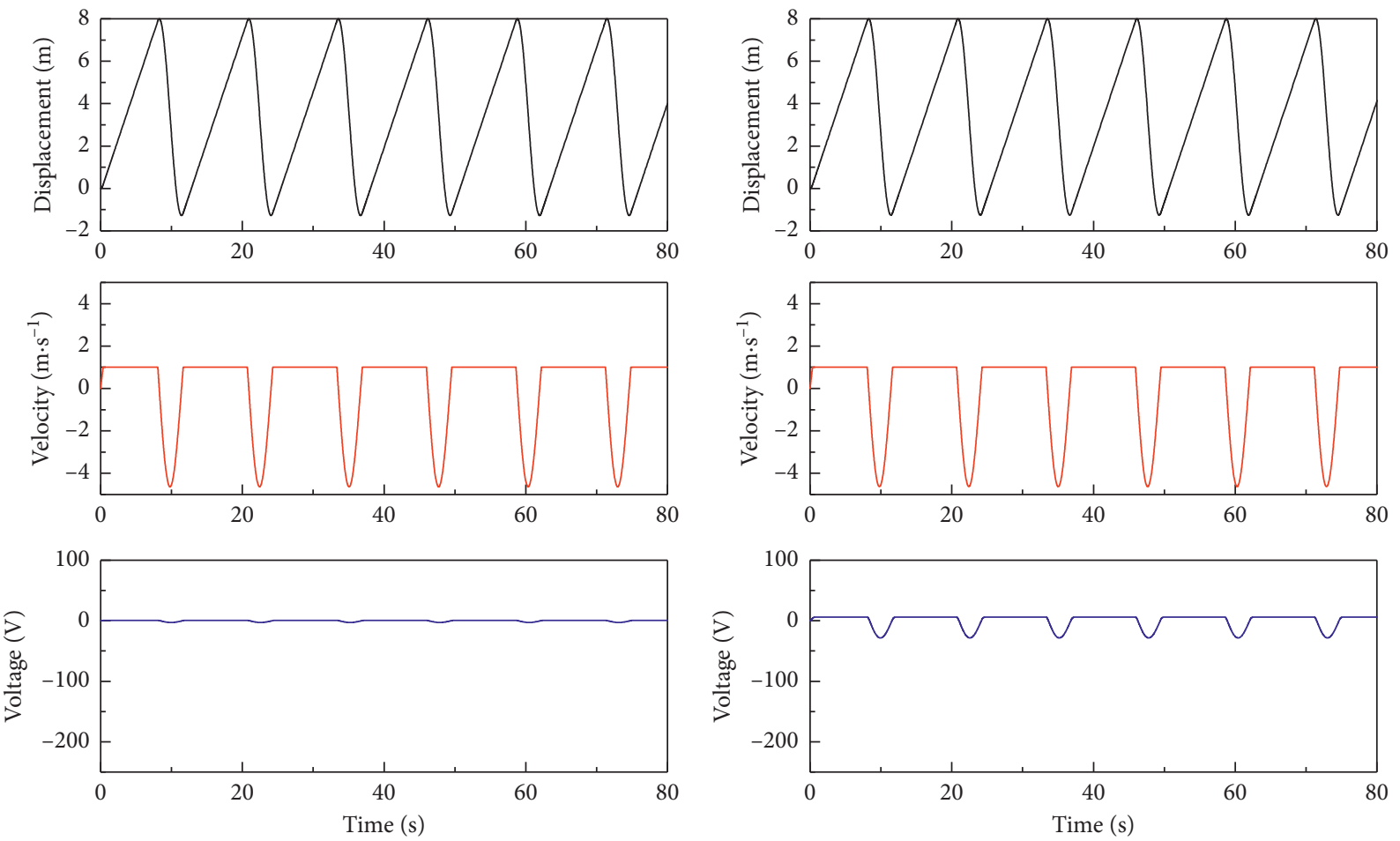

(a)

(b)
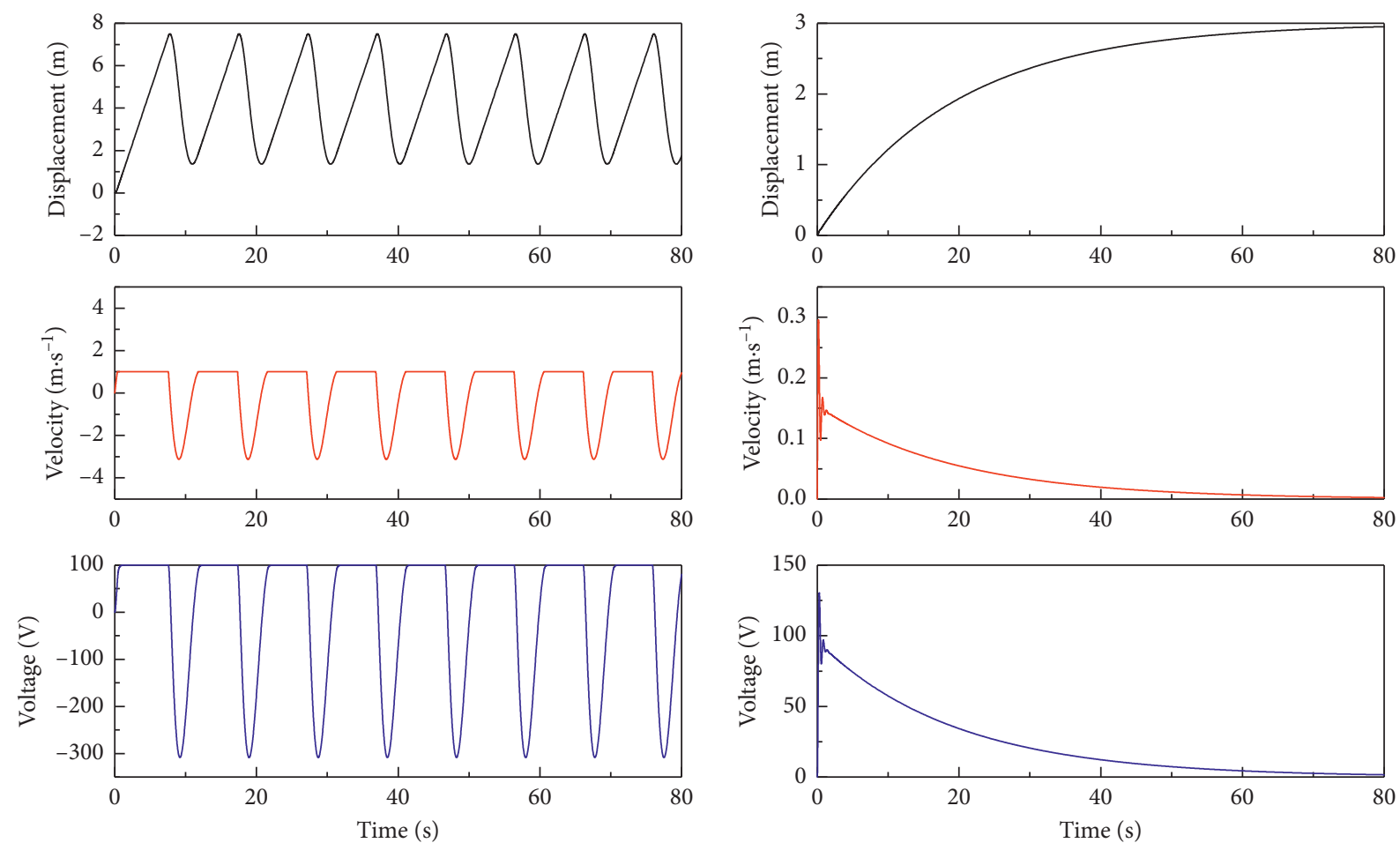

(c)

(d)

Figure 13: The displacement, velocity, and output voltage of the friction system when the force factor of piezoelectric element is $\alpha=3.1 \times 10^{-5} \mathrm{~N} / \mathrm{V}(\mathrm{a}), \alpha=3.1 \times 10^{-4} \mathrm{~N} / \mathrm{V}$ (b), $\alpha=5 \times 10^{-3} \mathrm{~N} / \mathrm{V}$ (c), and $\alpha=3.1 \times 10^{-2} \mathrm{~N} / \mathrm{V}$ (d) in the friction law I.

to ascend firstly and then descend with the continuous increase of the force factor, suggesting that there exists an optimized force factor which gives the highest harvested energy and conversion efficiency. This is because when the force factor exceeds a certain value, the vibration of the friction system will be suppressed; thus, the total harvested energy and conversion efficiency start to decrease with the further increase of the force factor. 

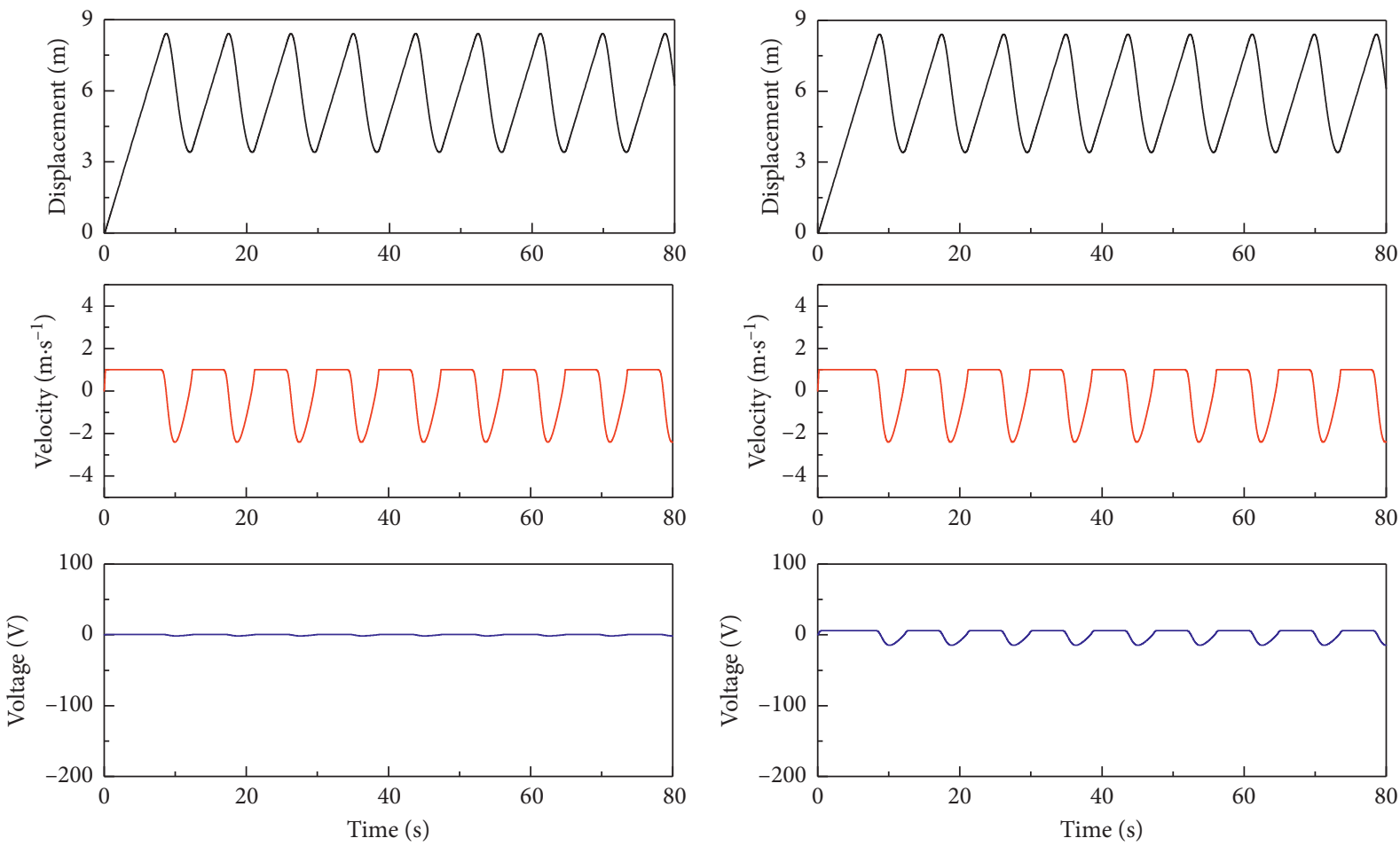

(a)

(b)
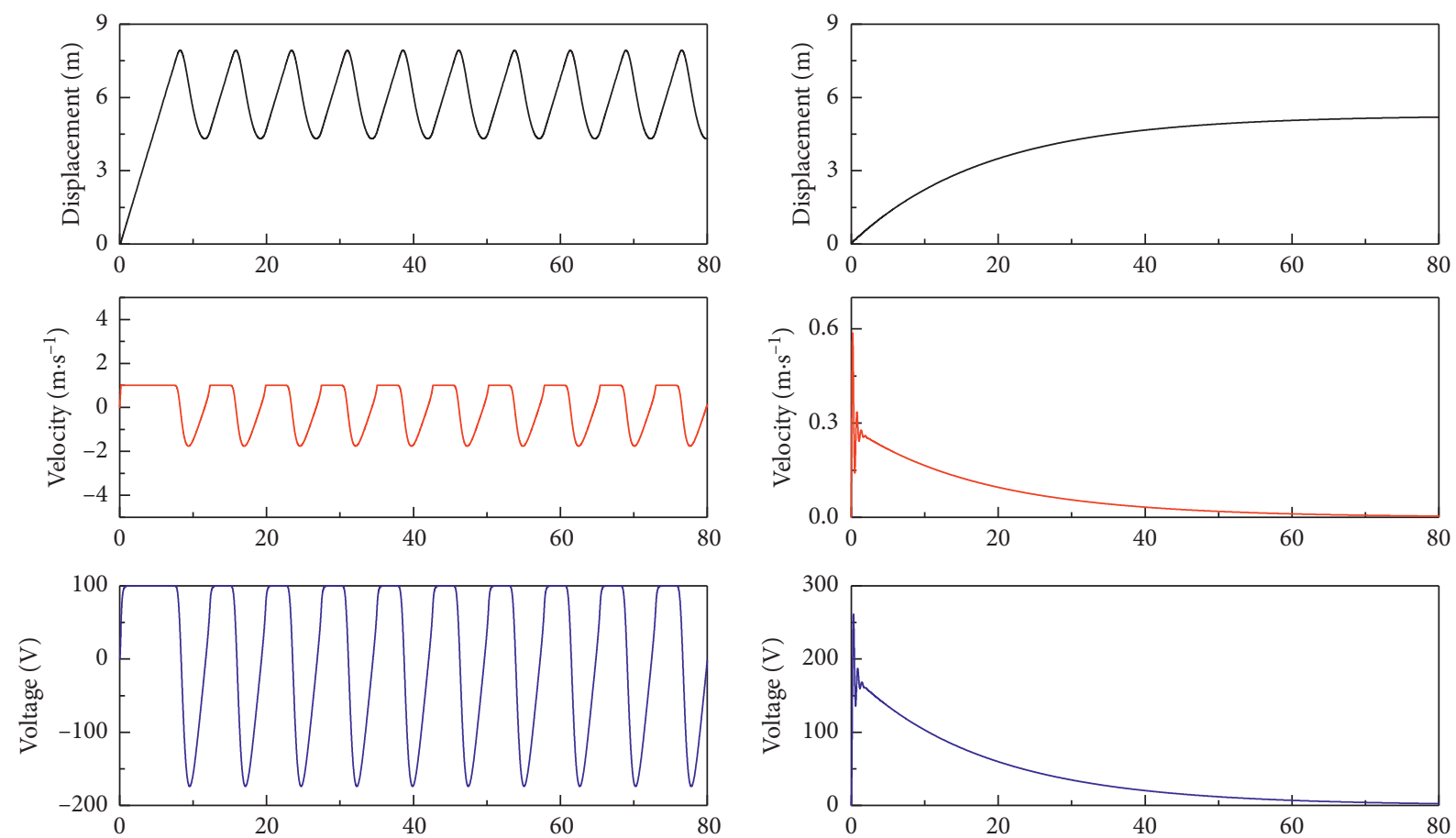

(c)

(d)

Figure 14: The displacement, velocity, and output voltage of the friction system when the force factor of piezoelectric element is $\alpha=3.1 \times 10^{-5} \mathrm{~N} / \mathrm{V}$ (a), $\alpha=3.1 \times 10^{-4} \mathrm{~N} / \mathrm{V}$ (b), $\alpha=5 \times 10^{-3} \mathrm{~N} / \mathrm{V}$ (c), and $\alpha=3.1 \times 10^{-2} \mathrm{~N} / \mathrm{V}$ (d) in the friction law II.

\section{Numerical Analysis of a Two-Degree-of- Freedom Friction System}

3.1. Two-Degree-of-Freedom Friction System. Some reported and published studies [52-54] are focused on the energy harvesting via stick-slip friction-induced vibration. However, in the real applications, the stick-slip vibration can be switched to mode coupling instability due to the variation of system parameters [55]. Thus, in this section, a two-degreeof-freedom friction system which is able to cause mode 


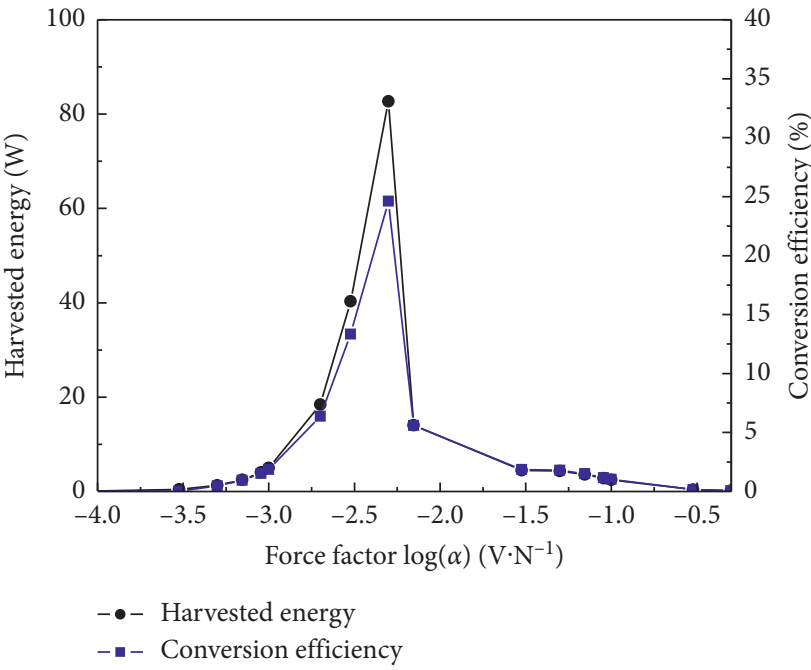

(a)

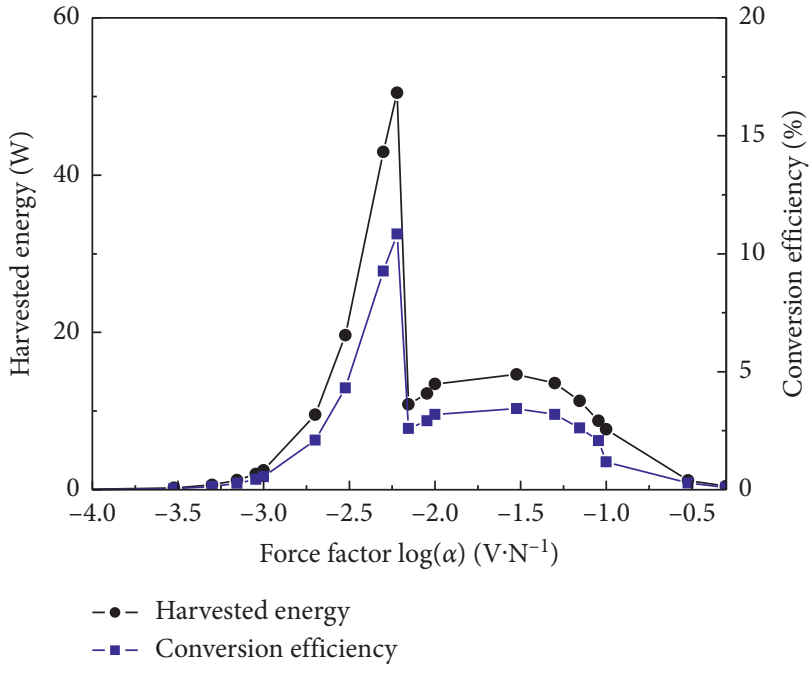

(b)

FIGURE 15: The harvested energy and conversion efficiency of the friction system with different force factors in the friction laws I (a) and II (b).

coupling instability is established, as shown in Figure 16. Similar to the one-degree-of-freedom friction system shown in Figure 1, a normal load $F$ is imposed to $m$ in the downward direction to make contact with the moving rigid belt. The belt is sliding at a constant velocity $v_{0} . m$ is linked with a linear spring $k_{1}$ and a damper $c_{1}$ in the horizontal direction and a grounded damper $c_{2}$ in the vertical direction. To couple the normal and tangential vibration, an oblique spring $k_{3}$, which has an inclination angle of 45 degrees to the horizontal direction, is connected with $m$. The contact stiffness between $m$ and the belt is represented by a linear spring $k_{2}$ and a nonlinear cubic spring $k_{n l}$. The horizontal and normal directions are defined as the $x$-axis and $y$-axis, respectively. The position of zero point is defined as the position before $F$ is imposed onto the $m$. The mode coupling instability of the two-degree-of-freedom friction system is due to the asymmetry of the stiffness matrix caused by friction; thus, the friction coefficient between the friction pairs is assumed to be a constant value $\mu$.

During vibration, the contact force $F_{N}$ between $m$ and the belt can be expressed as

$$
F_{N}=-k_{2} y-k_{n l} y^{3} \text {. }
$$

In addition, two piezoelectric elements, denoted as Piezo-1 and Piezo-2, respectively, are linked with $m$ in both directions, which are used to convert the vibration energy in both directions into electric energy. Without them, this friction system is identical to the model studied by Li et al. [56].

The mechanical and piezoelectric coupling equation of friction system can be written as

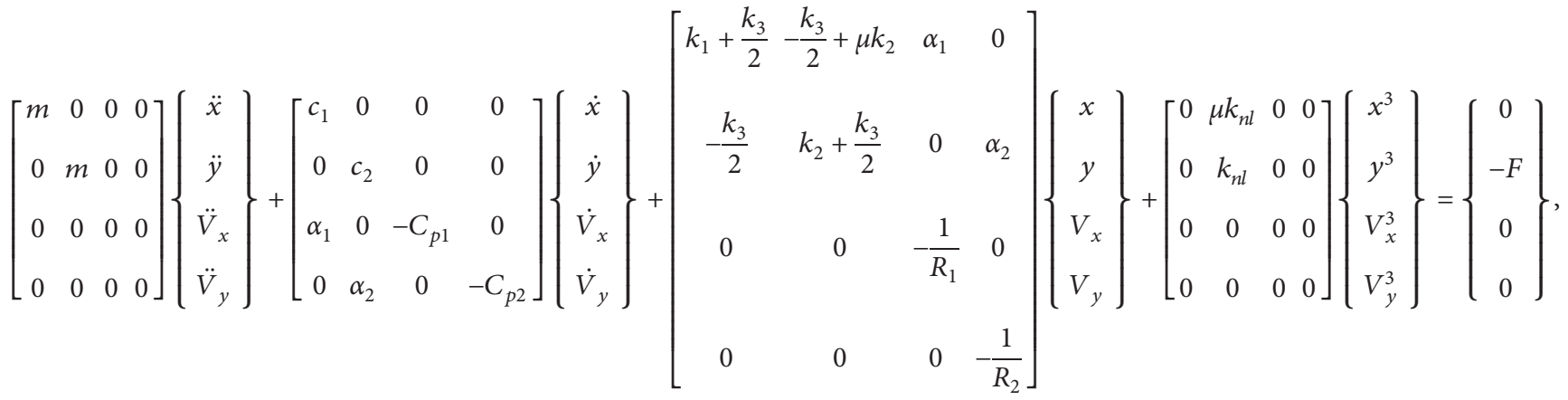

where $\alpha_{1}$ and $\alpha_{2}$ are the force factors of Piezo-1 and Piezo-2, respectively; $C_{p 1}$ and $C_{p 2}$ are the clamped capacitances of Piezo-1 and Piezo-2, respectively; and $V_{x}$ and $V_{y}$ are the output voltages of Piezo-1 and Piezo-2, respectively. It is worth noting that equation (11) is valid for the situation that $m$ remains in contact with the belt during vibration, i.e., contact force $F_{N}>0$. Once $m$ jumps up and separates from the belt because of vibration, both the contact springs $k_{2}$ and $k_{n l}$ will cease to contribute their stiffness to the mass as illustrated in Figure 17. At this moment, a new equation of motion will be formed as follows: 


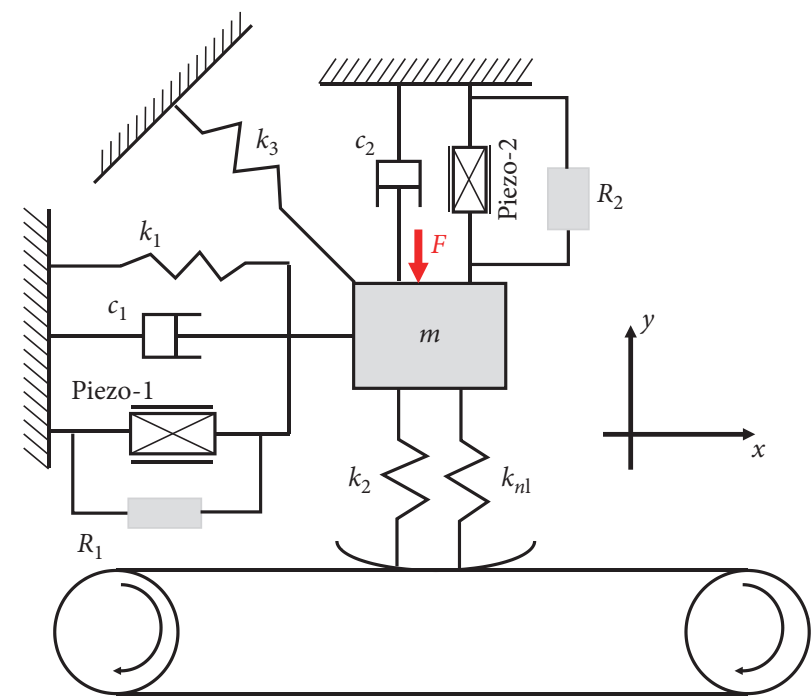

FIgURE 16: Two-degree-of-freedom friction system with piezoelectric elements.

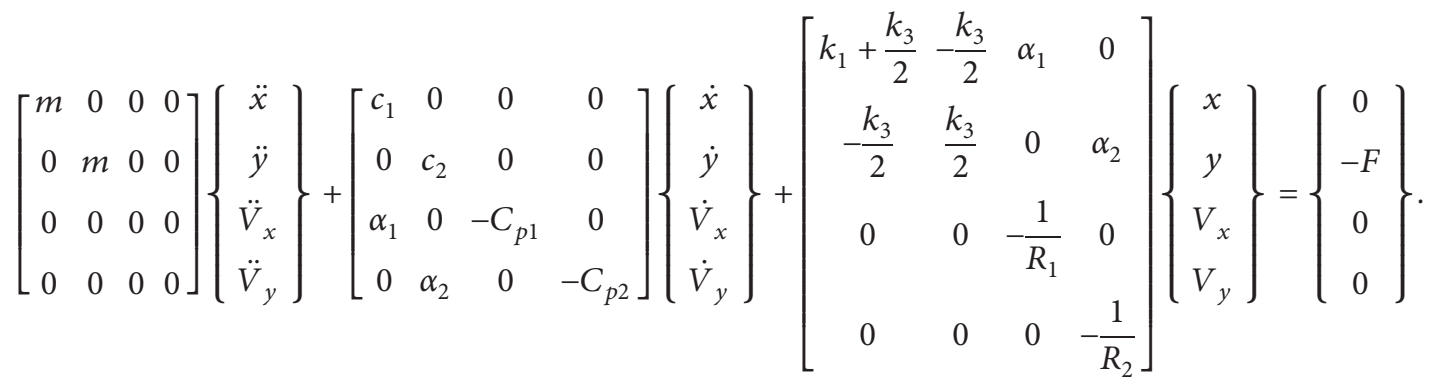

In the literature, a majority of research on this or similar models ignores the separation behaviour between $m$ and the belt during friction-induced vibration. However, the separation behaviour between the friction pairs plays a significant role in modifying the dynamics of the friction system [56], which consequently results in the variation of the energy harvesting performance. Therefore, in the following transient dynamic analysis of this friction system, both cases, i.e., ignoring separation and considering separation between $m$ and the belt, will be analyzed and discussed.

\subsection{Stability Analysis}

3.2.1. Equilibrium Points and Jacobian Matrix. The stability analysis is applied to equation (11). The equilibrium points $\left(x_{e}, y_{e}, V_{x e}\right.$, and $\left.V_{y e}\right)$ of the system can be determined by solving equation (11) with the first and second derivatives of these four variables taken to be zero, which becomes

$$
\left[\begin{array}{cccc}
k_{1}+\frac{k_{3}}{2} & -\frac{k_{3}}{2}+u k_{2} & \alpha_{1} & 0 \\
-\frac{k_{3}}{2} & k_{2}+\frac{k_{3}}{2} & 0 & \alpha_{2} \\
0 & 0 & -\frac{1}{R_{1}} & 0 \\
0 & 0 & 0 & -\frac{1}{R_{2}}
\end{array}\right]\left\{\begin{array}{c}
x_{e} \\
y_{e} \\
V_{x e} \\
V_{y e}
\end{array}\right\}+\left[\begin{array}{cccc}
0 & u k_{n l} & 0 & 0 \\
0 & k_{n l} & 0 & 0 \\
0 & 0 & 0 & 0 \\
0 & 0 & 0 & 0
\end{array}\right]\left\{\begin{array}{c}
x_{e}^{3} \\
y_{e}^{3} \\
V_{x e}^{3} \\
V_{y e}^{3}
\end{array}\right\}=\left\{\begin{array}{c}
0 \\
-F \\
0 \\
0
\end{array}\right\}
$$

There are three roots of equation (13), but only one root which is real number and has physical meaning is used in the following analysis. Defining the transformation relationships as $P_{1}=x, P_{2}=\dot{x}, P_{3}=y, P_{4}=\dot{y}, P_{5}=V_{1}$, and $P_{6}=V_{2}$. Thus, equation (11) is converted into a set of first-order differential equations as follows: 


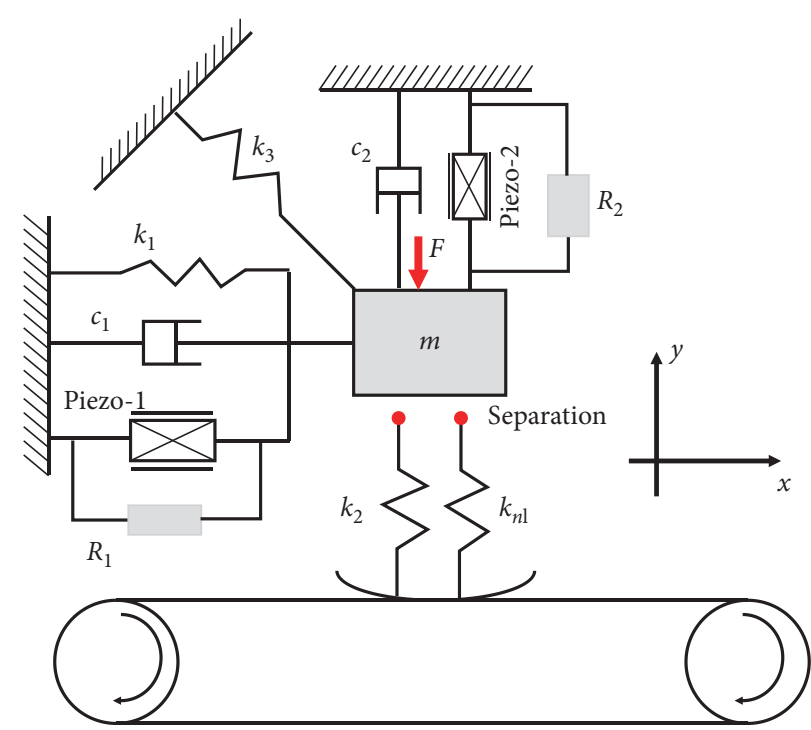

FIGURE 17: The situation that mass separates from the sliding belt.

$$
\left[\begin{array}{l}
\dot{P}_{1} \\
\dot{P}_{2} \\
\dot{P}_{3} \\
\dot{P}_{4} \\
\dot{P}_{5} \\
\dot{P}_{6}
\end{array}\right]=\left[\begin{array}{ccccccc}
0 & 1 & 0 & 0 & 0 & 0 \\
-\frac{k_{1}+\left(k_{3} / 2\right)}{M} & -\frac{c_{1}}{M} & -\frac{\mu k_{2}-\left(k_{3} / 2\right)}{M} & 0 & -\frac{\alpha_{1}}{M} & 0 \\
0 & 0 & 0 & 1 & 0 & 0 \\
\frac{k_{3} / 2}{M} & 0 & -\frac{k_{2}+\left(k_{3} / 2\right)}{M} & -\frac{c_{2}}{M} & 0 & -\frac{\alpha_{2}}{M} \\
0 & \frac{\alpha_{1}}{C_{p 1}} & 0 & 0 & -\frac{1}{R_{1} C_{p 1}} & 0 \\
0 & 0 & 0 & \frac{\alpha_{2}}{C_{p 2}} & 0 & -\frac{1}{R_{2} C_{p 2}}
\end{array}\right]\left[\begin{array}{c}
P_{1} \\
P_{2} \\
P_{3} \\
P_{4} \\
P_{5} \\
P_{6}
\end{array}\right]+\left[\begin{array}{cccccc}
0 & 0 & 0 & 0 & 0 & 0 \\
0 & 0 & -\frac{\mu k_{n l}}{M} & 0 & 0 & 0 \\
0 & 0 & 0 & 0 & 0 & 0 \\
0 & 0 & -\frac{k_{n l}}{M} & 0 & 0 & 0 \\
0 & 0 & 0 & 0 & 0 & 0 \\
0 & 0 & 0 & 0 & 0
\end{array}\right]\left[\begin{array}{c}
P_{1}^{3} \\
P_{2}^{3} \\
P_{3}^{3} \\
P_{4}^{3} \\
P_{5}^{3} \\
P_{6}^{3}
\end{array}\right]+\left[\begin{array}{c}
0 \\
0 \\
0 \\
F \\
-\frac{F}{M} \\
0 \\
0
\end{array}\right] .
$$

The Jacobian matrix can be derived via expanding nonlinear equation (14) in a first-order truncated Taylor series at the equilibrium point, written as follows:

$$
J=\left[\begin{array}{cccccc}
0 & 1 & 0 & 0 & 0 & 0 \\
-\frac{k_{1}+\left(k_{3} / 2\right)}{M} & -\frac{c_{1}}{M}-\frac{\mu k_{2}-\left(k_{3} / 2\right)}{M}-\frac{3 \mu k_{n l}}{M} y_{e}^{2} & 0 & -\frac{\alpha_{1}}{M} & 0 \\
0 & 0 & 0 & 1 & 0 & 0 \\
\frac{k_{3} / 2}{M} & 0 & -\frac{k_{2}+\left(k_{3} / 2\right)}{M}-\frac{3 k_{n l}}{M} y_{e}^{2} & -\frac{c_{2}}{M} & 0 & -\frac{\alpha_{2}}{M} \\
0 & \frac{\alpha_{1}}{C_{p 1}} & 0 & 0 & -\frac{1}{R_{1} C_{p 1}} & 0 \\
0 & 0 & 0 & \frac{\alpha_{2}}{C_{p 2}} & 0 & -\frac{1}{R_{2} C_{p 2}}
\end{array}\right] .
$$


The stability of the system can be evaluated by calculating the eigenvalues $\lambda$ of the Jacobian matrix [57]. These eigenvalues reflect the features of the local stability at the equilibrium point. If any real part of the eigenvalues is positive, the system is regarded as unstable. In the following sections, the effect of different working parameters on the stability of the system will be systemically studied.

\subsubsection{Effect of Normal Load on the Stability of the Friction}

System. Eigenvalue analysis of the friction system at various normal loads is carried out. The parameters set in this analysis are $m=5 \mathrm{~kg}, k_{1}=100 \mathrm{~N} / \mathrm{m}, k_{2}=50 \mathrm{~N} / \mathrm{m}, k_{3}=60 \mathrm{~N} / \mathrm{m}$, $k_{n l}=100 \mathrm{~N} / \mathrm{m}^{3}, \quad c_{1}=c_{2}=0.32 \mathrm{~N} /(\mathrm{m} / \mathrm{s}), \alpha_{1}=\alpha_{2}=3.1 \times 10^{-5} \mathrm{~N} /$ $\mathrm{V}, C_{p 1}=C_{p 2}=7.2 \times 10^{-6} \mathrm{~F}$, and $R_{1}=R_{2}=20000 \Omega$. Results are shown in Figure 18. With the increase of normal load, the critical friction coefficient for the generation of friction-induced vibration decreases at first and then increases (Figure 18(a)), suggesting that the friction system has a stronger tendency to generate unstable vibration at first and then the tendency gradually reduces. Figure 18(b) plots the critical friction coefficients of the friction system under different normal loads. The critical friction coefficient gradually decreases and then starts to increase as long as the normal load reaches a certain value. This phenomenon indicates that the unstable region increases first and then decreases. From the view of energy harvesting by means of friction-induced vibration, a wider region of unstable vibration is beneficial for energy harvesting.

\subsubsection{Effect of External Electric Resistance on the Stability of} the Friction System. Figure 19 shows the eigenvalue of the friction system when the external electric resistance varies over a large range of $5000 \Omega$ to $500000 \Omega$. This range is considered to be used in many studies of vibration energy harvesting [9]. The values of springs and dampers are identical to those used in Section 3.2.2, and the normal load is set as $70 \mathrm{~N}$. It is found that when $\alpha=3.1 \times 10^{-5} \mathrm{~N} / \mathrm{V}$, the variation of external electric resistance has little effect on the stability of friction system, in which the stable region remains almost unchanged regardless of the values of external electric resistance, as shown in Figure 19(a). For the imaginary parts, no visible change can be found with the variation of external electric resistance, as shown in Figure 19(b). This is caused by the relative lower value of the force factor. In the transient dynamic analysis, this phenomenon will be further verified by calculating the dynamic performance of the friction system in the time domain.

\subsubsection{Effect of Force Factor on the Stability of the Friction} System. Figure 20 illustrates the eigenvalue analysis results of the friction system at various force factors of the piezoelectric elements. The normal load is set as $70 \mathrm{~N}$ and $R_{1}=R_{2}=20000 \Omega$. With the increase of force factor, the critical friction coefficient for the friction system to generate unstable vibration gradually becomes larger, which indicates that the friction system has a relative lower tendency to generate unstable vibration at a larger force factor, as shown in Figure 20(a). For the imaginary parts, the coupling frequency shows significant change with the variation of force factor and a larger force factor causes a higher vibration frequency, as shown in Figure 20(b). It is worth noting that although the vibration tendency of the friction system is reduced with the increase of force factor, the variation of output voltage may not follow this trend, considering that the piezoelectric elements with larger force factor have a stronger potential to convert vibration energy to electrical energy at higher force factor values. Therefore, transient dynamic analysis will be accordingly performed in the following section to further detect the variation of dynamics and output voltages with time.

\subsection{Transient Dynamic Analysis}

3.3.1. Influence of the Normal Load. In the transient dynamic analysis, the fourth-order Runge-Kutta method is used to calculate the transient dynamic responses of the friction system in the time domain. Two situations of the friction system during sliding process is taken into consideration, i.e., ignoring and considering the separation between the mass and belt during vibration. Their dynamic behaviours and output voltages will be analyzed in the following analysis.

In the case of ignoring the separation, the Runge-Kutta method can directly solve the dynamic response of the friction system described by the equations of motion of equation (11) through stepping integration. While in the case of considering the separation, the calculation process becomes more complicated. During the contact process, the equation of motion is given by equation (11); then, the contact force $F_{N}$ is estimated at the end of each time step; once the contact force $F_{N}$ is estimated below zero, suggesting that the mass is separated from the belt, and the critical point corresponding to the moment when the contact force $F_{N}$ becomes zero is determined. At this moment, the equation of motion is changed to equation (12). The associated motion state (displacement, velocity, and acceleration) previously obtained at this critical point is used as the initial value of equation (12). During the separation process, the displacement of the mass is detected at the end of each time step, as long as the displacement is detected to be below zero; the critical point when the mass comes back to the belt is determined and the equation of motion switches back to equation (11) until the next separation. In this work, the impact between the belt and the mass is ignored as it is believed to have a minor influence.

Figure 21 shows the dynamics and output voltages of the friction system with different normal loads when ignoring separation. Constant friction coefficient $\mu=0.4$ and external electric resistance $R=20000 \Omega$ are set in this analysis. The values of springs and dampers are identical to those in the previous analysis. No visible sustained vibration and voltage 


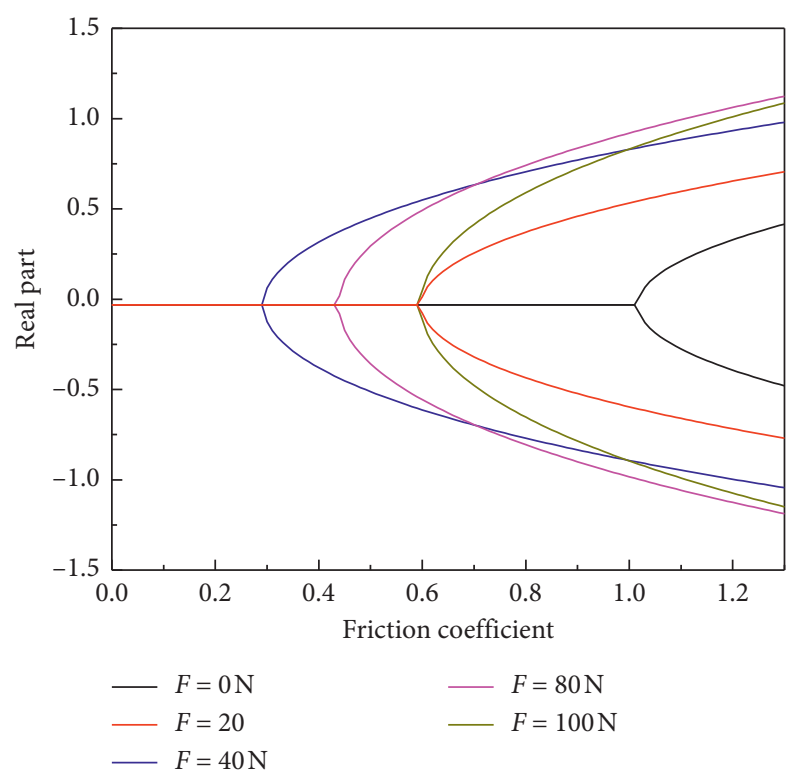

(a)

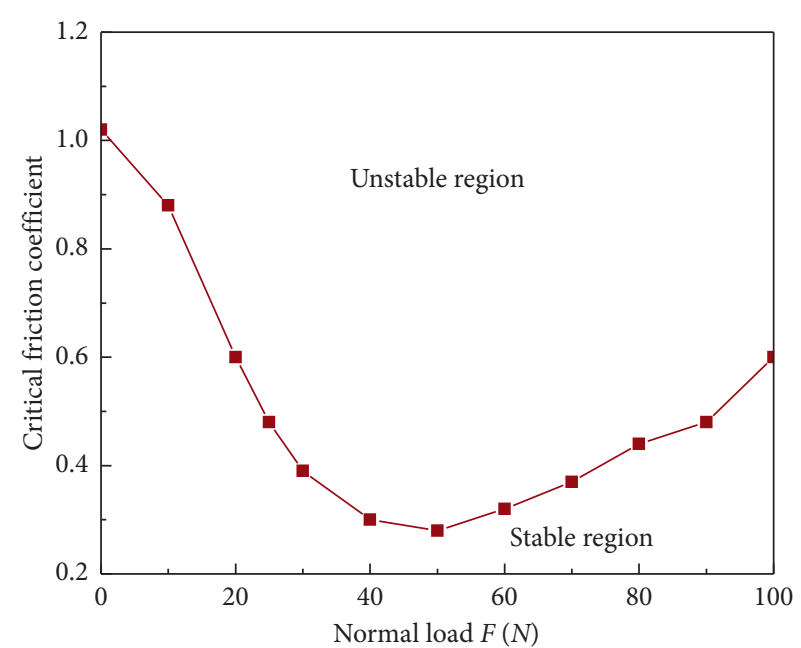

(b)

Figure 18: The real parts (a) and the critical friction coefficients (b) of the friction system at different normal loads.

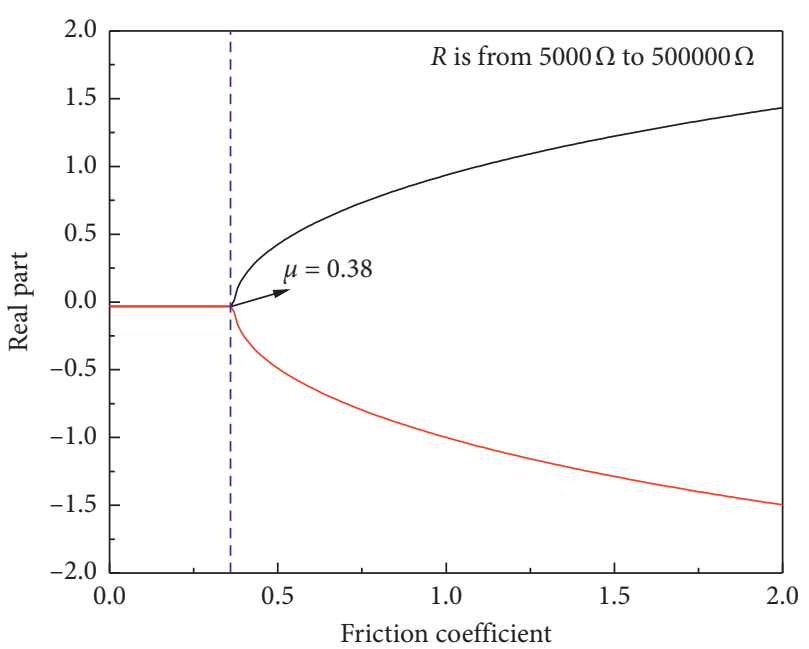

(a)

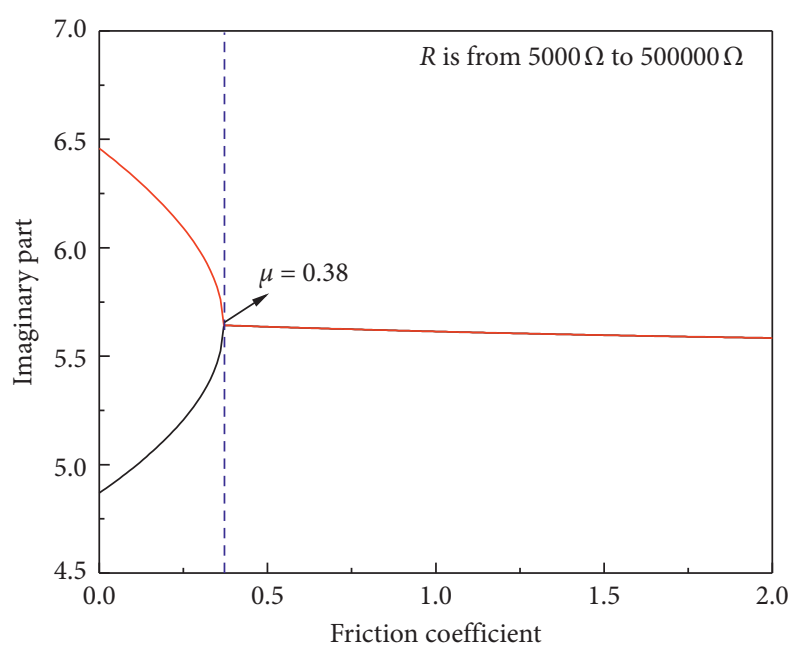

(b)

FIgURE 19: The real parts (a) and imaginary parts (b) of the friction system with the variation of external electric resistances.

output can be observed at a low normal load. With the normal load increasing to a certain value, strong self-excited vibration appears and consequently visible voltage output is found to be generated in both directions. While with the normal load further increasing, the vibration shows a considerable decrease and no visible voltage output can be detected. This phenomenon indicates that the normal load can significantly affect the vibration behaviour of the friction system and accordingly the energy harvesting performance. This result further verifies the CEA analysis results shown in Figure 18. A larger normal is beneficial for generating the mode lock-in between the normal and tangential directions; thus, the vibration signals show a significant increase when increasing the normal load. With further increasing the normal load, mode lock-out will happen, and the vibration reduces significantly. Apparently, there is a critical normal load value for the generation of the strongest vibration and the highest voltage output.

A similar trend of the vibration and output voltage performance in the case of considering separation can be observed in Figure 22. A critical normal load value for the highest amplitude of vibration and output voltage can be observed as well. In addition, it is found that when separation is considered, the amplitudes of both the vibration 


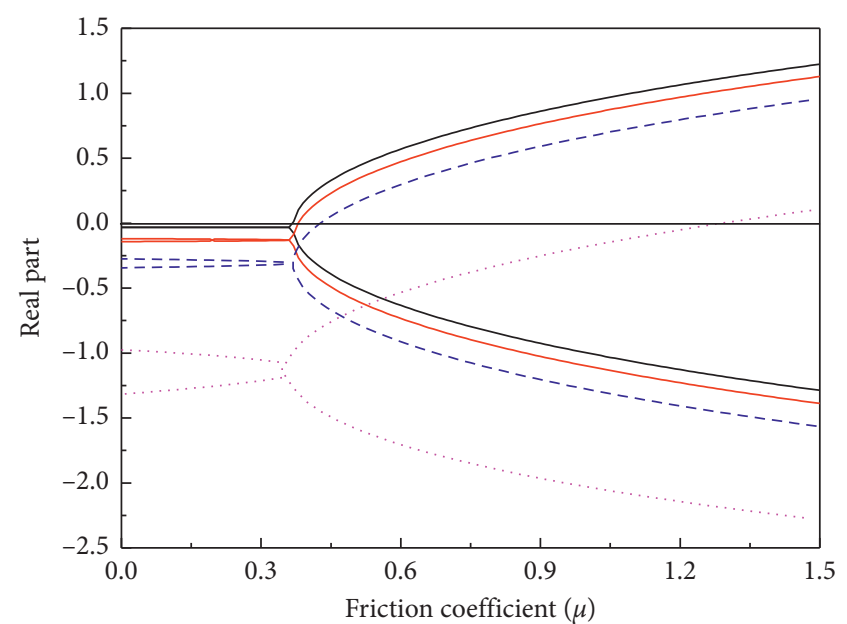

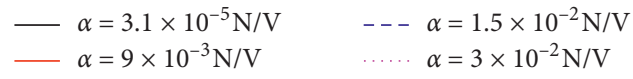

(a)

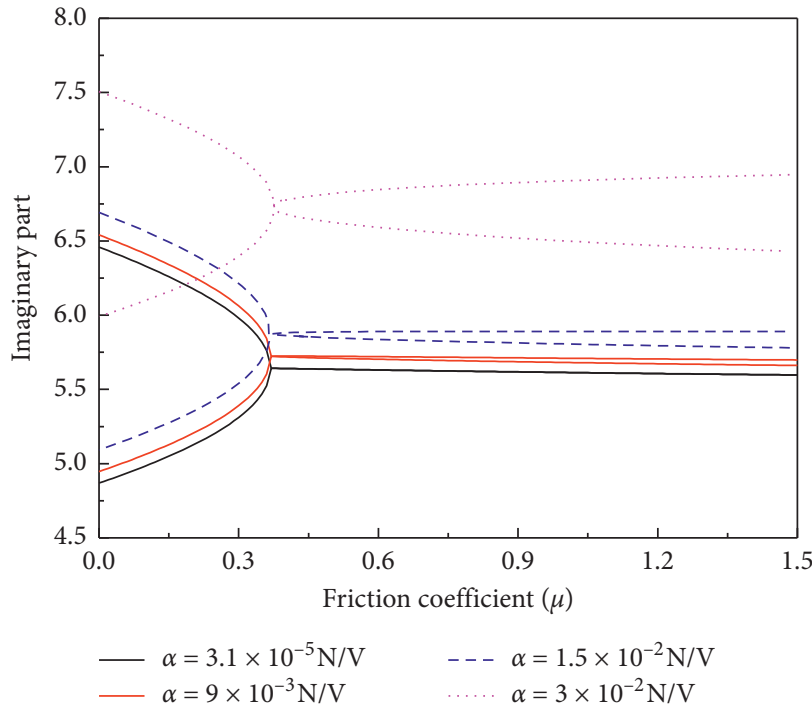

(b)

Figure 20: The real parts (a) and imaginary parts (b) of the friction system with different force factors of piezoelectric elements.
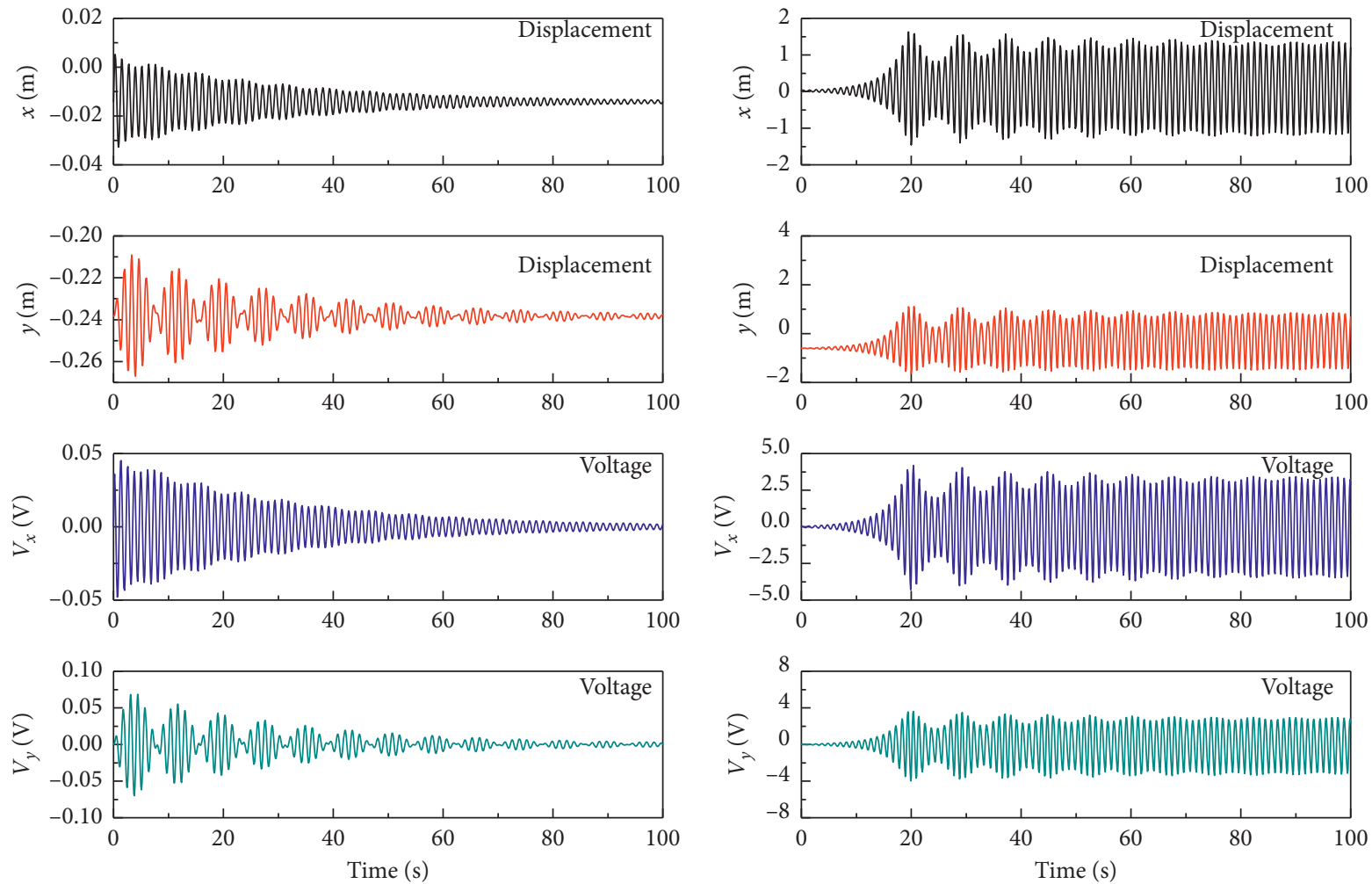

(a)

(b)

Figure 21: Continued. 

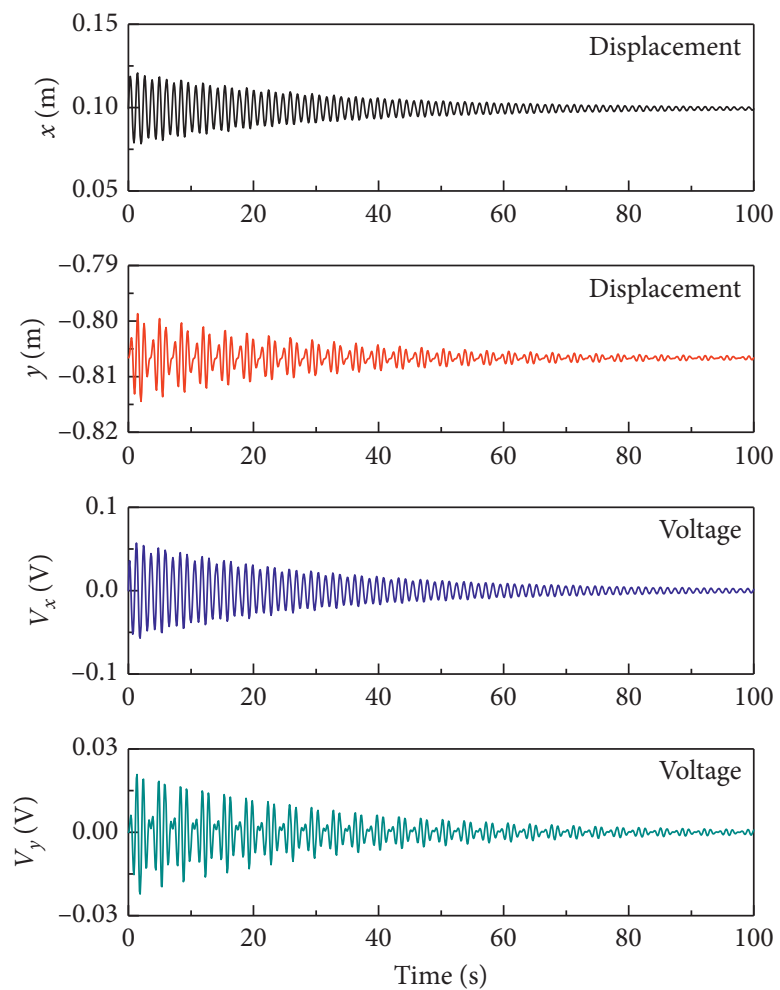

(c)

FIGURE 21: Ignoring separation, the displacement and output voltage of the friction system when the normal load is $F=20 \mathrm{~N}$ (a), $F=70 \mathrm{~N}$ (b), and $F=120 \mathrm{~N}$ (c).
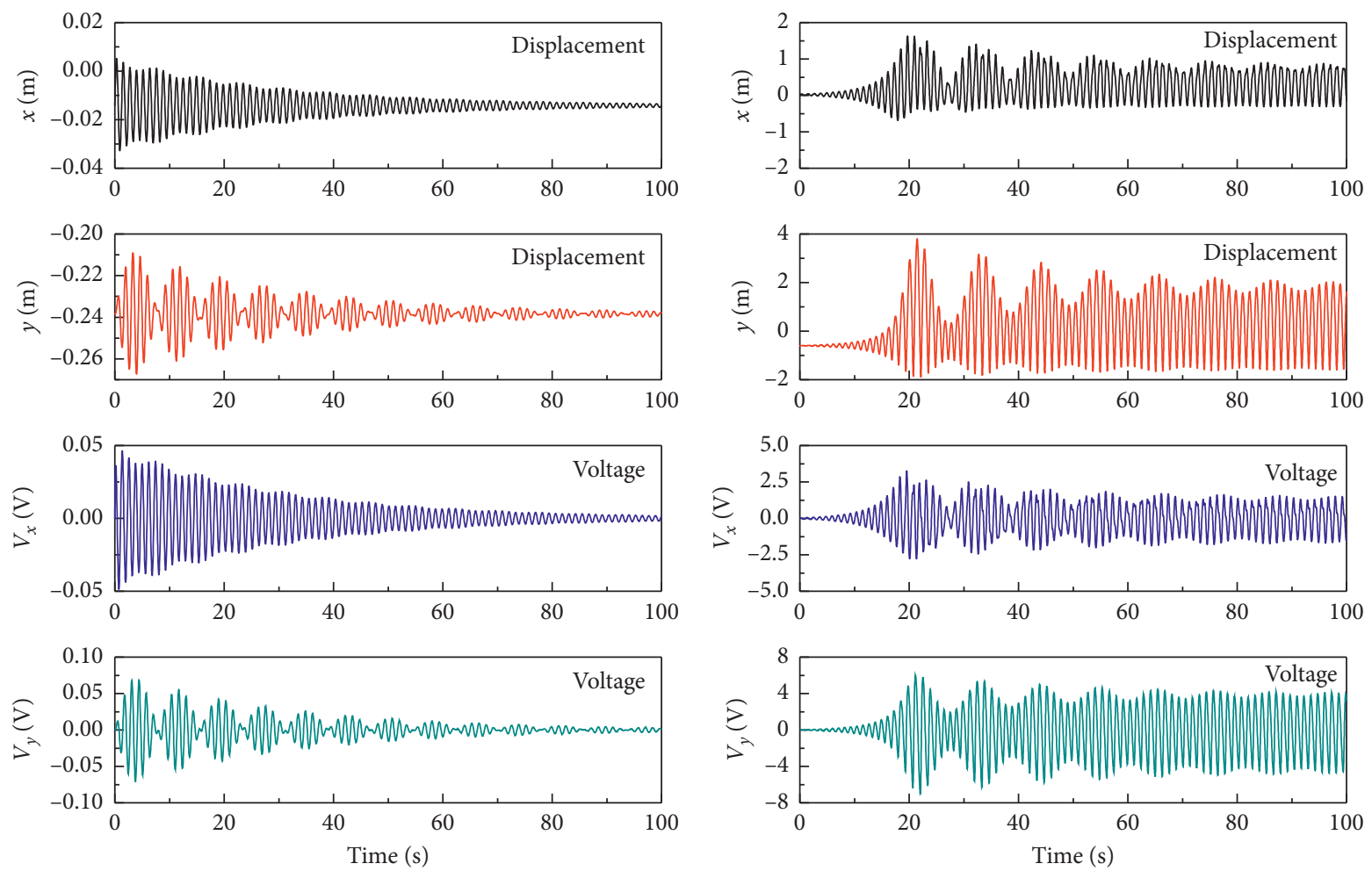

(a)

(b)

Figure 22: Continued. 

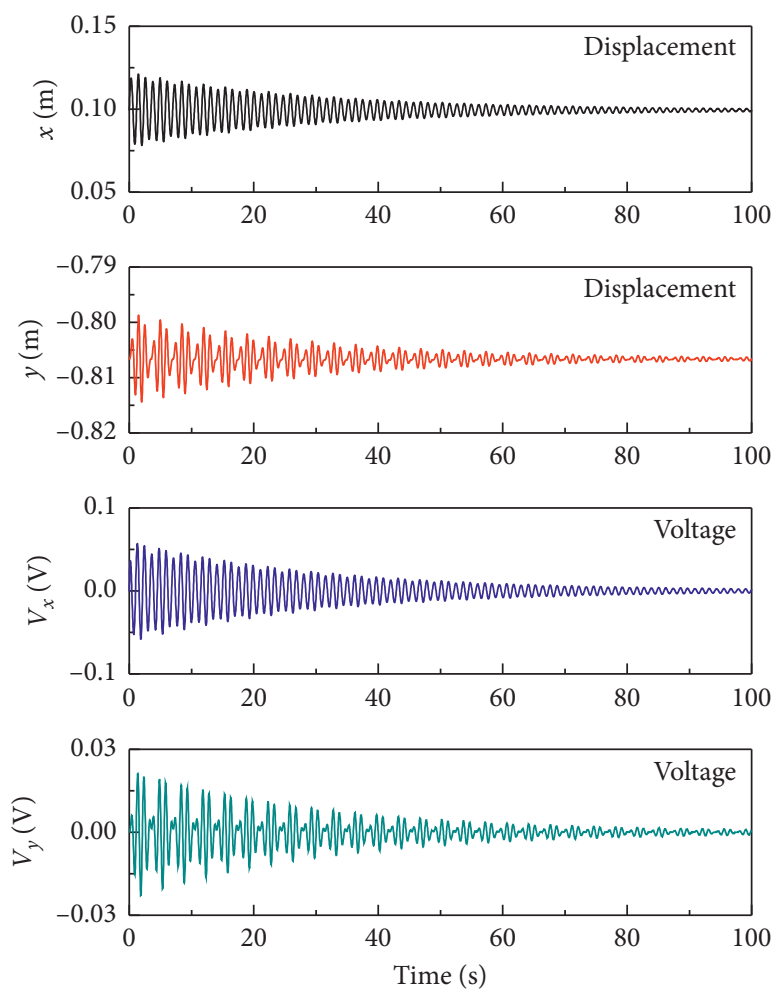

(c)

Figure 22: Considering separation, the displacement and output voltage of the friction system when the normal load is $F=20 \mathrm{~N}$ (a), $F=70 \mathrm{~N}$ (b), and $F=120 \mathrm{~N}(\mathrm{c})$.

and output voltage are considerably different from that case of ignoring separation. Visibly, the horizontal displacement and output voltage when considering separation is smaller compared with the situation of ignoring separation, while the normal displacement and output voltage show opposite results. This is because that the normal contact stiffness is lost during separation which results in the larger displacement in the normal direction, whilst the friction force disappears and will not make any contribution to the horizontal vibration.

The harvested energy value and the energy conversion efficiency of the friction system when ignoring separation are shown in Figure 23. For both the horizontal and normal directions, the harvested energy and conversion efficiency exhibit a sudden shift to a large value as long as the selfexcited vibration is sustained, further suggesting that the energy can be harvested via friction-induced vibration. With the increase of normal load, the harvested energy value and the energy conversion efficiency shows a considerable decrease once no visible vibration is generated from the system. In the vibration region, there is a critical load value to give the highest harvested energy and conversion efficiency.

When considering separation, as shown in Figure 24, there is still a critical load value for the generation of highest harvested energy and conversion efficiency, though the value is different from that calculated in the case of ignoring separation. Compared with the case of ignoring separation, the harvested energy and conversion efficiency gradually grow larger in the case of considering separation, and no sudden shift can be observed. In addition, when the normal load is above a certain value, both the harvested energy value and the energy conversion efficiency reduce significantly, since no visible vibration is generated from the friction system.

\subsubsection{Influence of the External Electrical Resistance.} Figure 25 illustrates the dynamic performance and output voltage of the friction system with different external electrical resistances when ignoring separation. Constant friction coefficient $\mu=0.4$ and normal load $F=70 \mathrm{~N}$ are used in this analysis. From the results of complex eigenvalues analysis shown in Figure 20, the variation of external electrical resistance will not affect the dynamic behaviour of the friction system and the friction system exhibits consistent vibration behaviour under different external electrical resistances. However, it is observed that external electrical resistance can visibly affect the amplitude of output voltages in both directions. The amplitudes of output voltages show a noticeable increase with the increase of external electrical resistance in a certain range. 


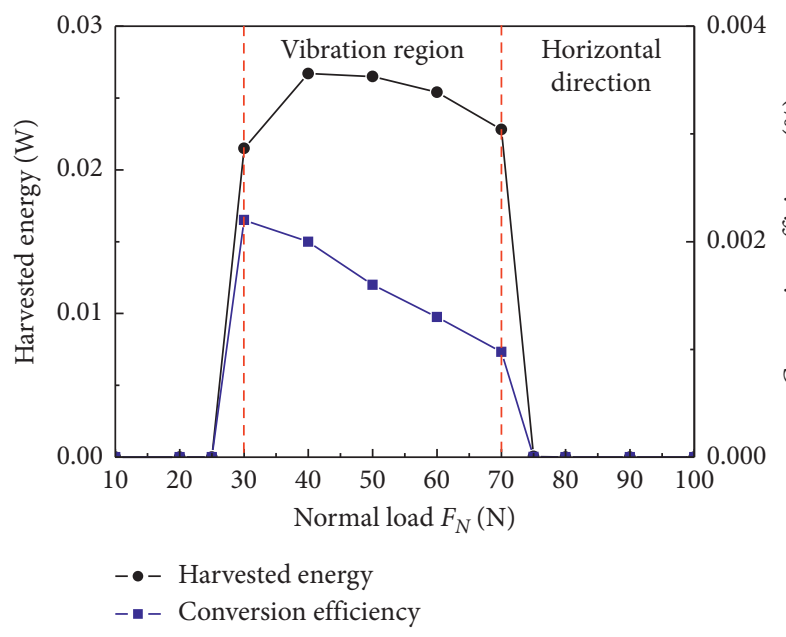

(a)

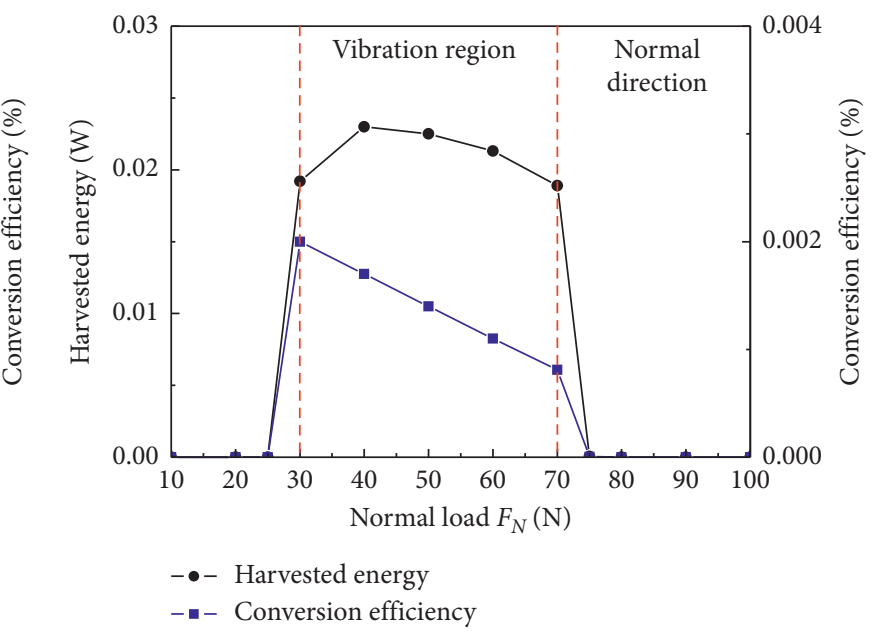

(b)

FIgURE 23: Ignoring separation, the harvested energy and conversion efficiency of the friction system in the horizontal direction (a) and normal direction (b) with different normal loads.

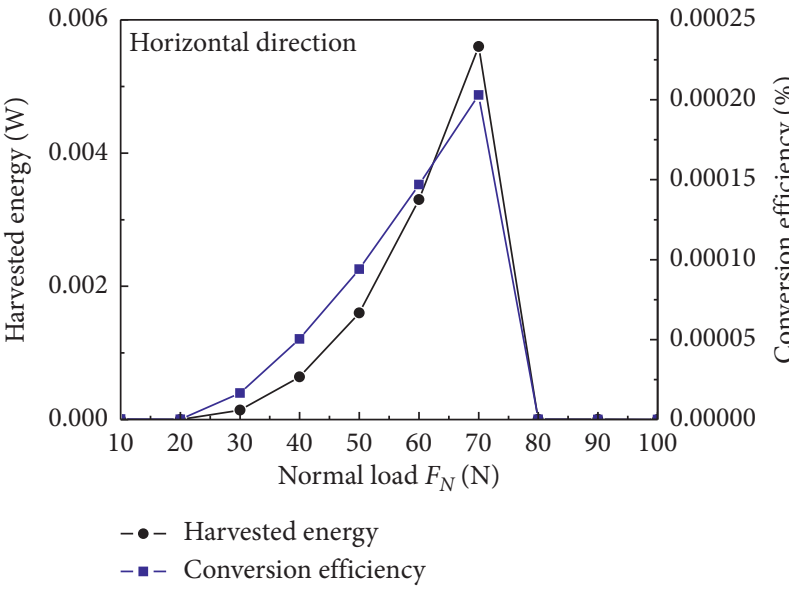

(a)

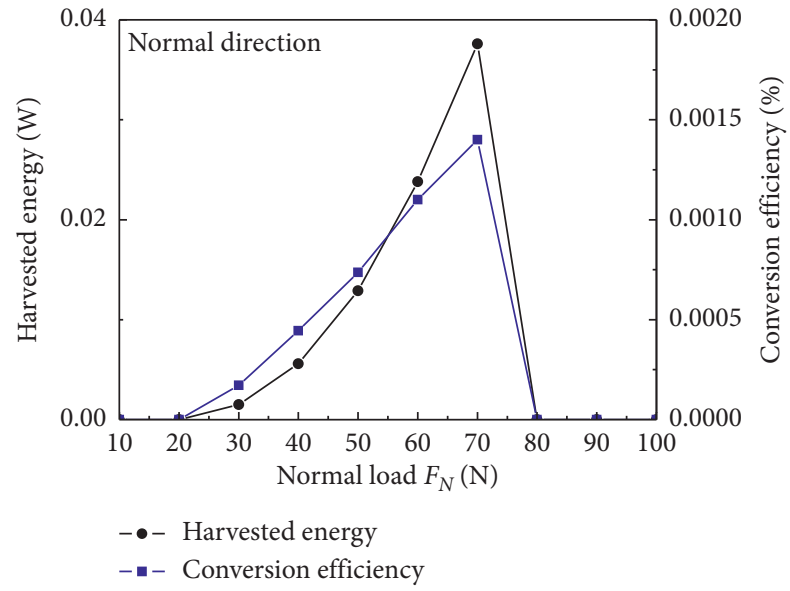

(b)

FIgURE 24: Considering separation, the harvested energy and conversion efficiency of the friction system in the horizontal direction (a) and normal direction (b) with different normal loads.

Thereafter, the output voltage will not exhibit a visible change with the further increase of external electrical resistance.

When the separation between the belt and the mass is taken into consideration, as shown in Figure 26, although the dynamic behaviour of the friction system is different from the case of ignoring separation, their results are qualitatively similar. Visibly, the variation of external electrical resistance will not significantly modify the vibration behaviour of the friction system; however, it can change the amplitude of output voltage. The amplitude of the output voltage will rise gradually and then remain steady with the continuous increase of the external electrical resistance.
When ignoring the separation between the contact pairs, the harvested energy value and the energy conversion efficiency of the friction system with different external electric resistances are illustrated in Figure 27. Both the harvested energy and conversion efficiency exhibit a tendency of increasing first and then decrease with the continuous increase of external electric resistances, suggesting that there is a critical resistance value to show the highest harvested energy and conversion efficiency. This similar tendency can be observed in the case of considering separation, as shown in Figure 28. Combining the results obtained from stability analysis and transient dynamic analysis of these two cases, it can be concluded that the external electric resistances has no 

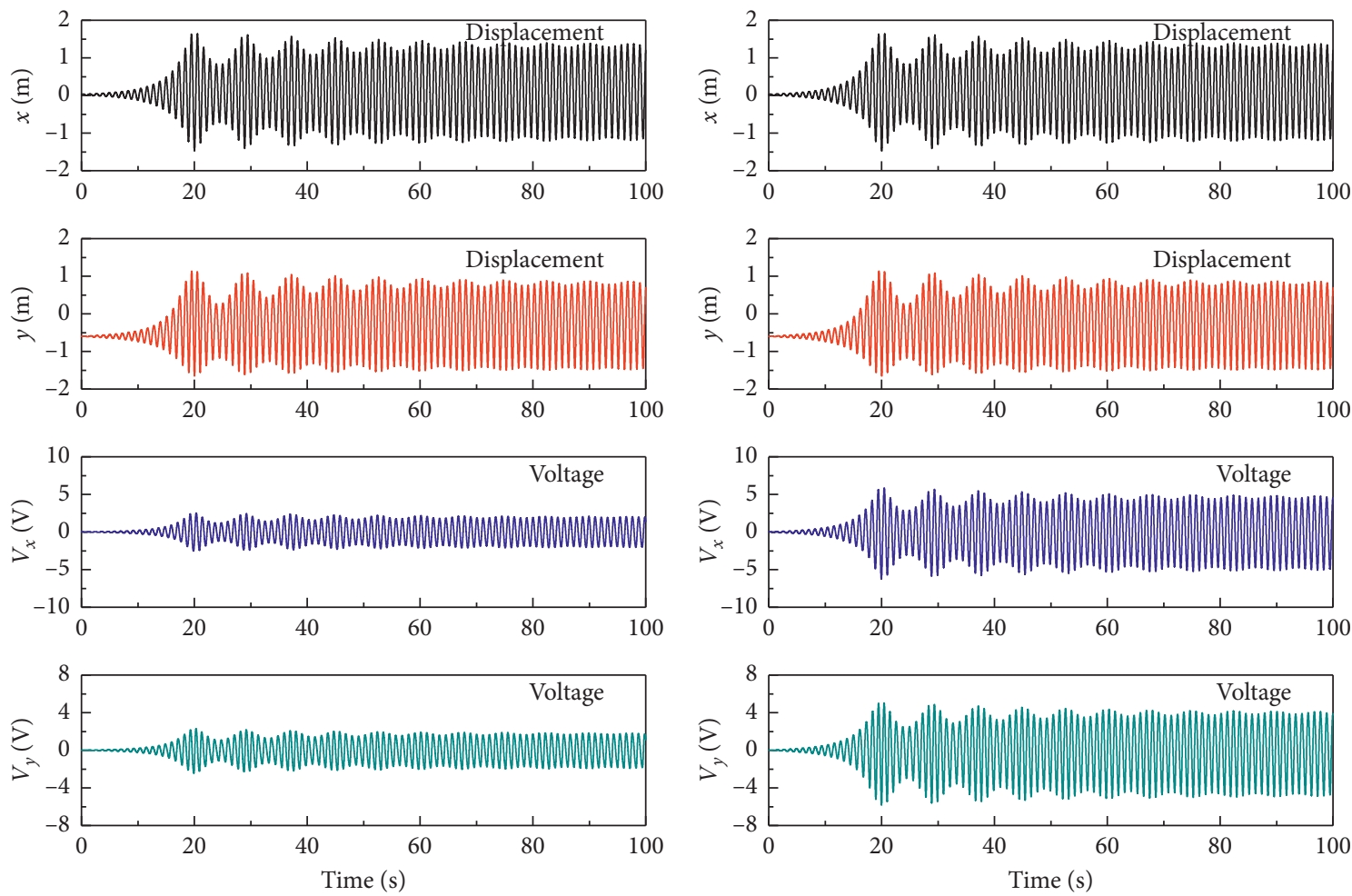

(a)

(b)
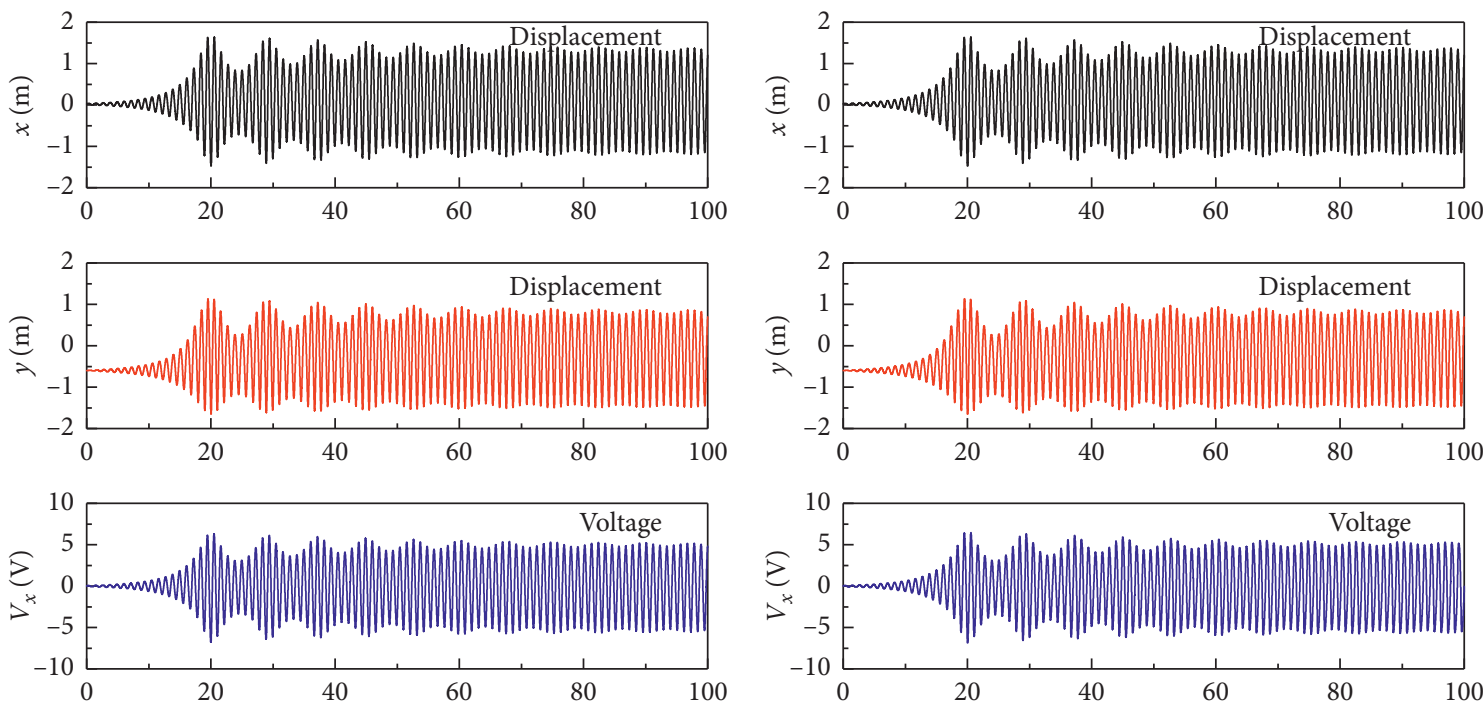

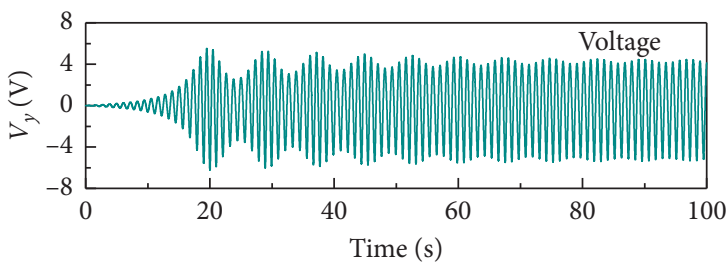

(c)

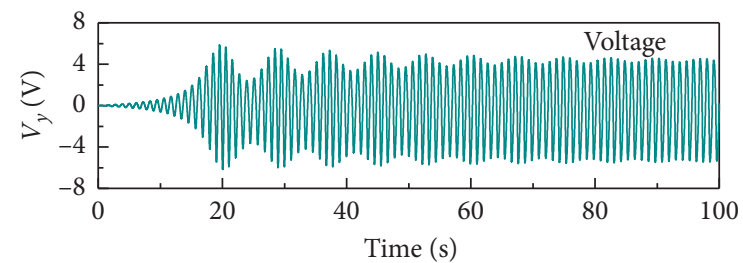

(d)

FIGURE 25: Ignoring separation, the displacement and output voltage of the friction system when the external electrical resistance is $R=10000 \Omega(\mathrm{a}), R=50000 \Omega(\mathrm{b}), R=100000 \Omega(\mathrm{c})$, and $R=200000 \Omega(\mathrm{d})$. 

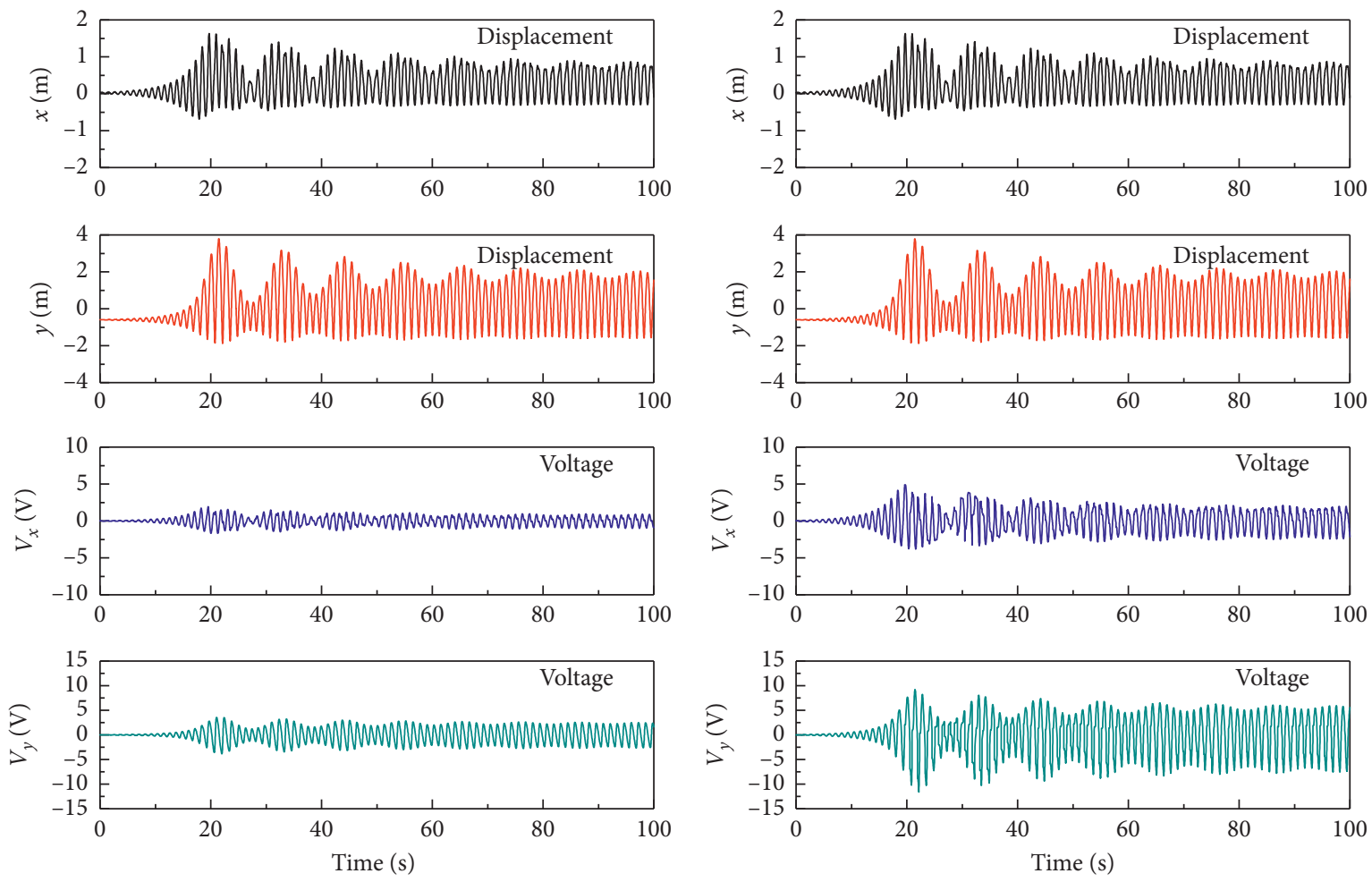

(a)

(b)
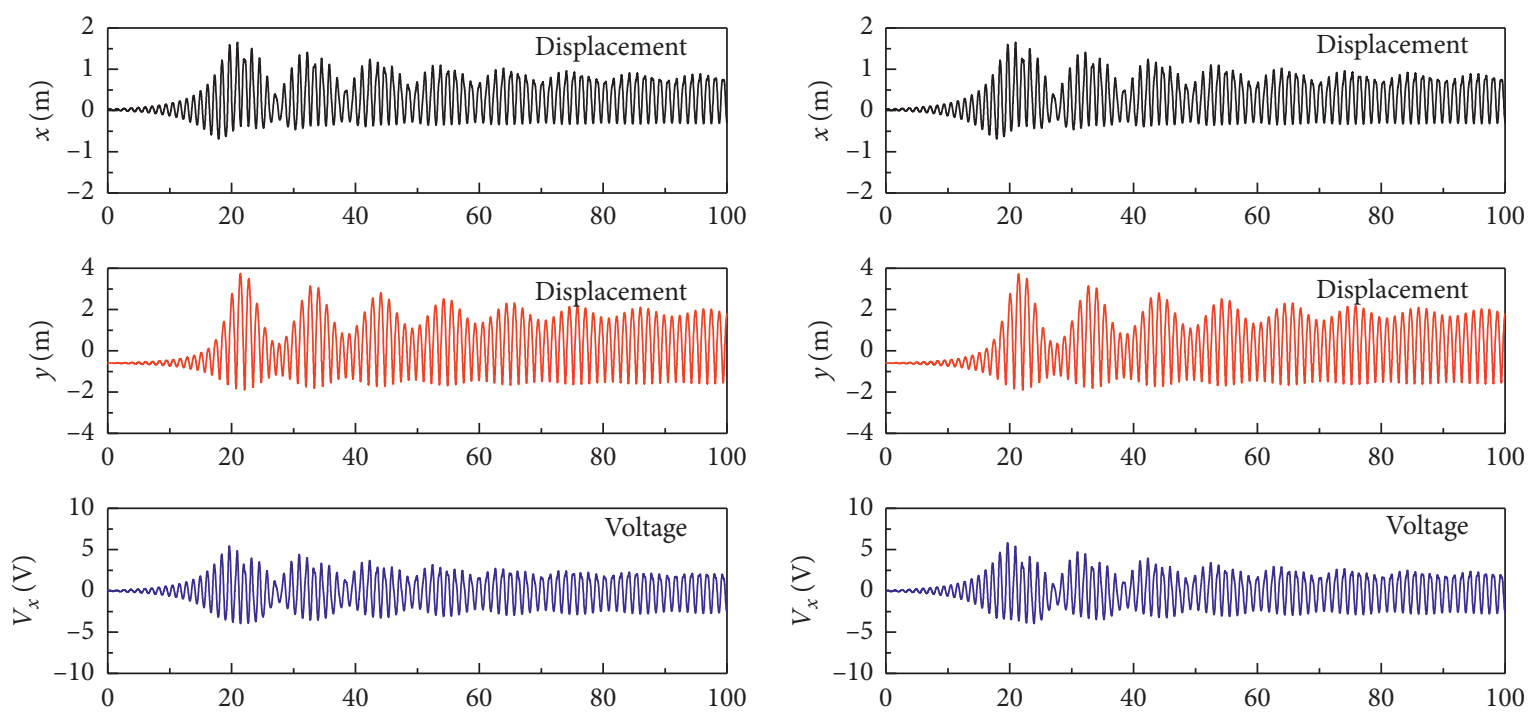

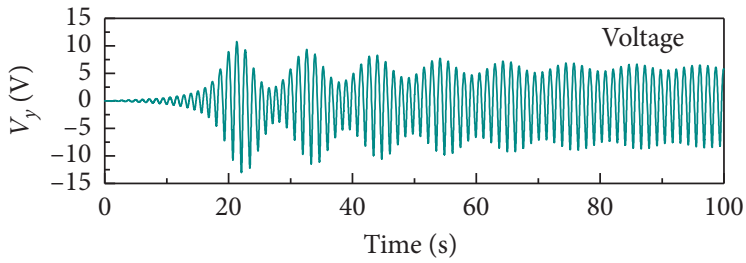

(c)

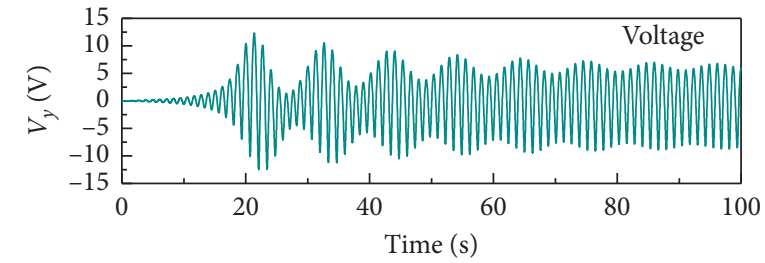

(d)

FIgURE 26: Considering separation, the displacement and output voltage of the friction system when the external electrical resistance is $R=10000 \Omega$ (a), $R=50000 \Omega$ (b), $R=100000 \Omega$ (c), and $R=200000 \Omega$ (d). 


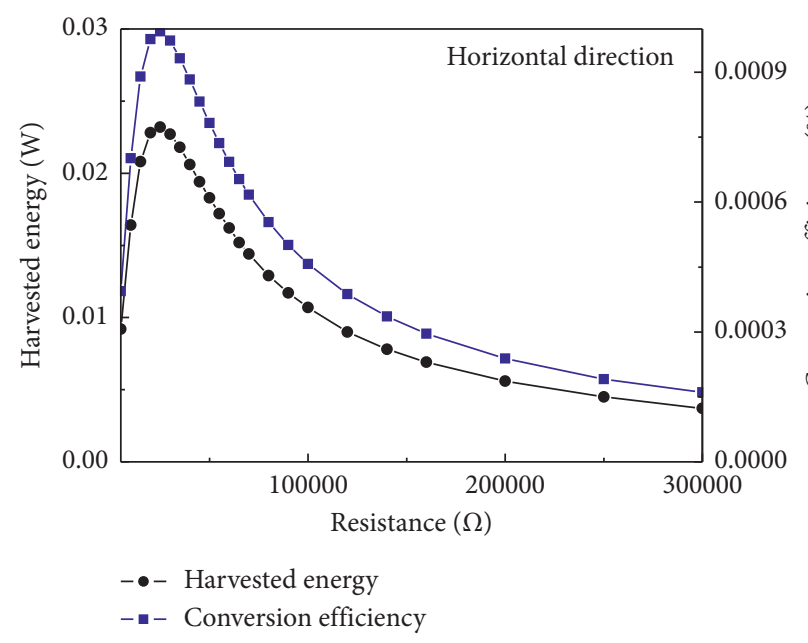

(a)

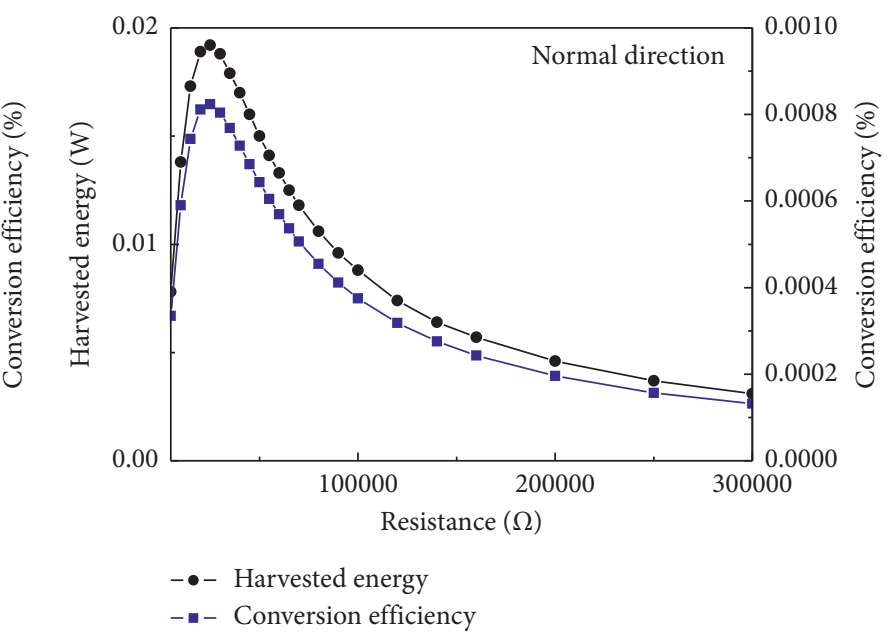

(b)

FIGURE 27: Ignoring separation, the harvested energy and conversion efficiency of the friction system in the horizontal direction (a) and normal direction (b) with different external electrical resistances.

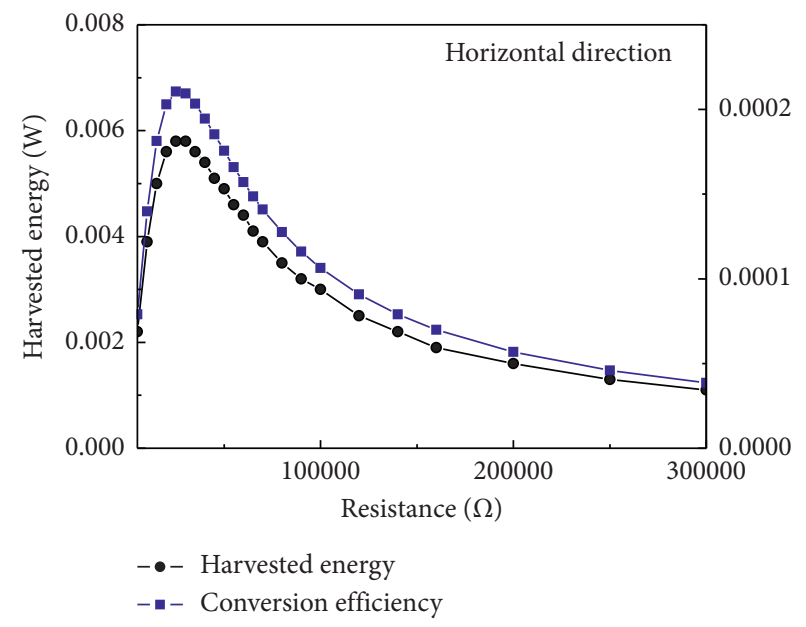

(a)

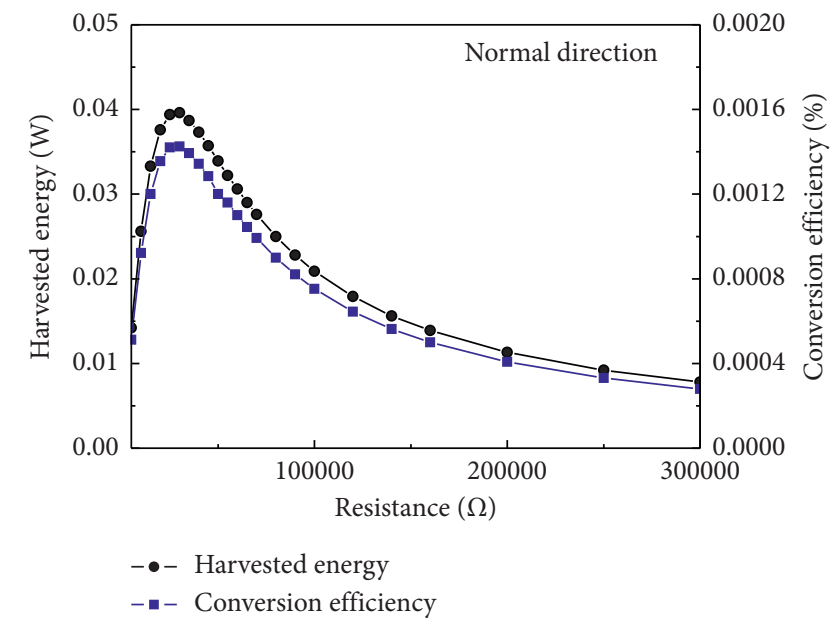

(b)

FIGURE 28: Considering separation, the harvested energy and conversion efficiency of the friction system in the horizontal direction (a) and normal direction (b) with different external electrical resistances.

effect on the dynamic behaviour of the friction system; however, it can significantly affect the output voltage amplitudes within limits, and an appropriate external electric resistances value is beneficial for obtaining the highest harvested energy and conversion efficiency. This phenomenon is qualitatively similar to that obtained from the stick-slip friction-induced vibration shown in Figure 14.

3.3.3. Influence of the Force Factor. Figures 29 and 30 illustrate the effect of force factor of the piezoelectric elements on the dynamics and output voltage. The normal load and friction coefficient used in this analysis are $70 \mathrm{~N}$ and 0.4 , respectively. It is seen that piezoelectric elements with a larger force factor can increase the output voltage in both situations. When the force factor exceeds a certain value, the vibration reduces drastically and thus the corresponding output voltages reduce significantly. Figures 31 and 32 show the harvested energy and the conversion efficiency of the friction system with the variation of force factor. The abscissa is expressed as the logarithmic form of force factor. For both cases of ignoring and considering separation, both the harvested energy and conversion efficiency show a tendency to increase firstly and then decrease with the increase of force factor, suggesting that there exists an optimized force factor which gives the highest harvested energy and conversion efficiency. This phenomenon is qualitatively similar to that obtained from the stick-slip friction-induced vibration shown in Figure 15. 

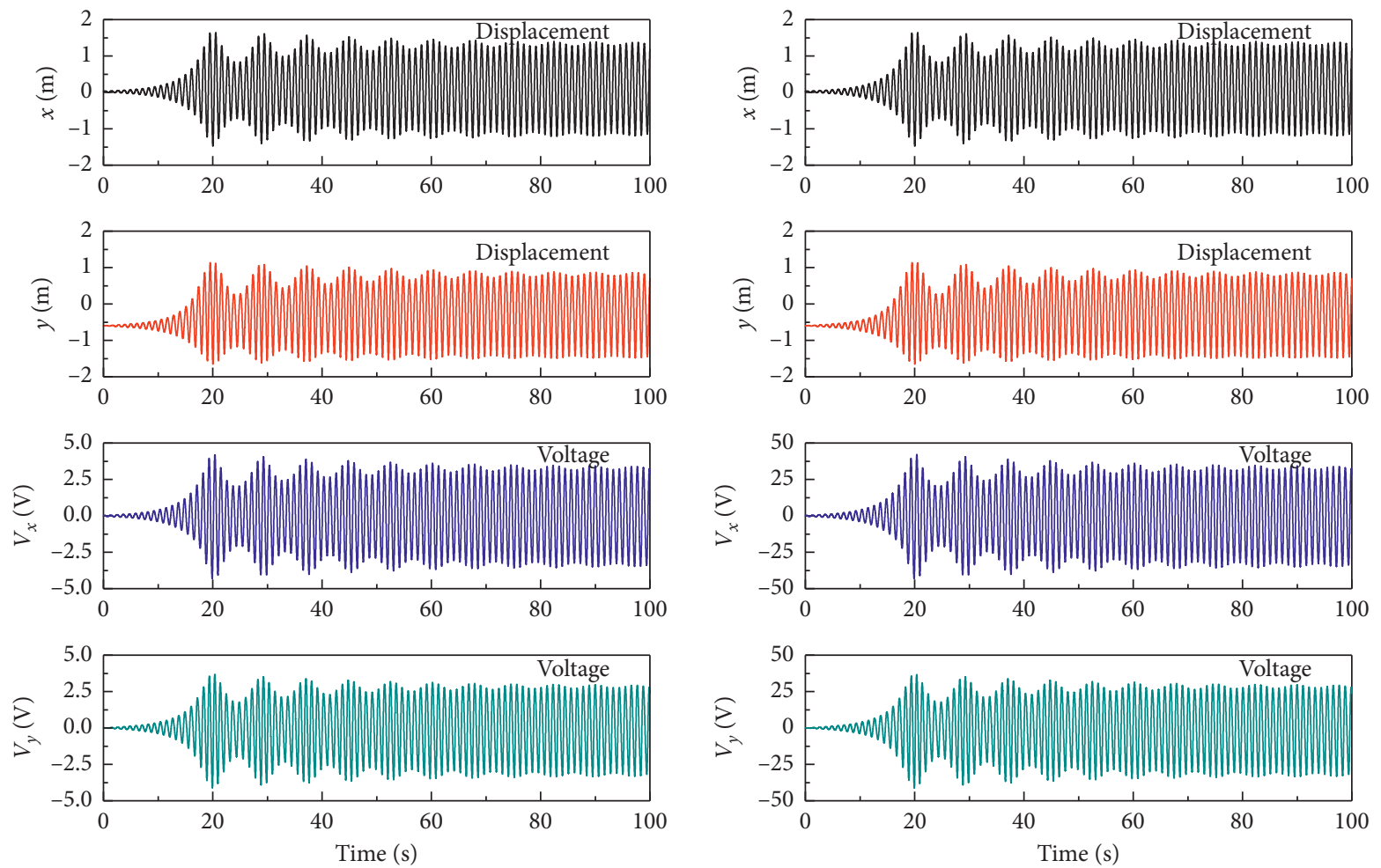

(a)

(b)
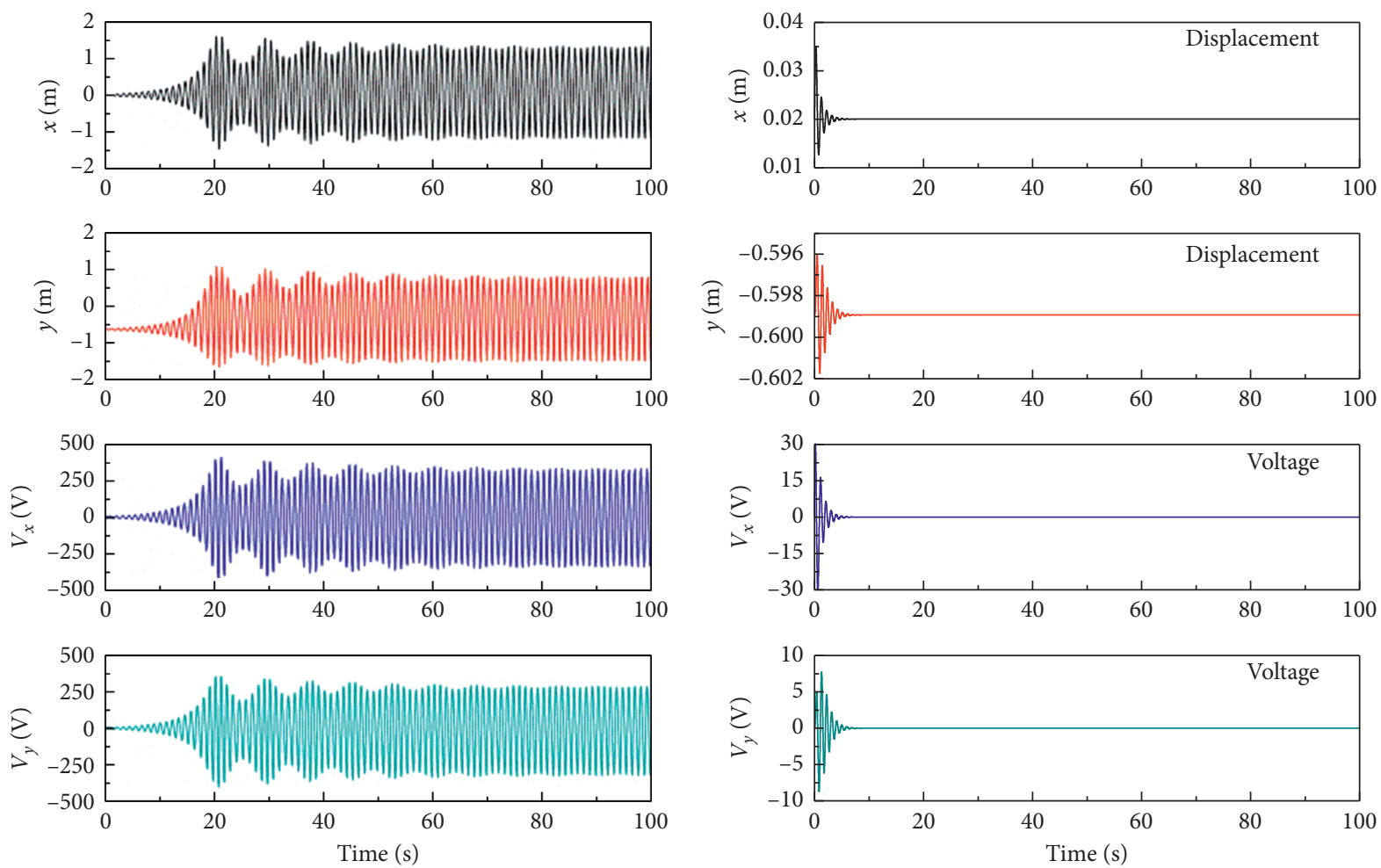

(c)

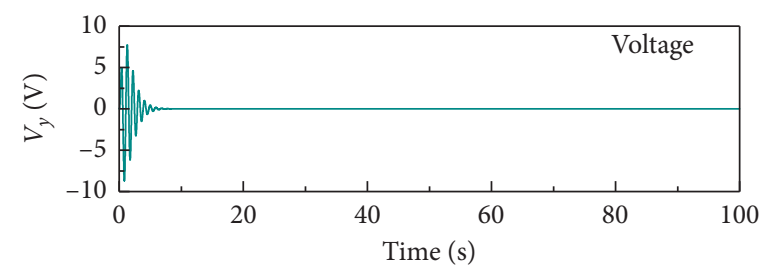

(d)

Figure 29: Ignoring separation, the displacements and output voltages of the friction system when the force factor of piezoelectric elements is $\alpha=3.1 \times 10^{-5} \mathrm{~N} / \mathrm{V}$ (a), $\alpha=3.1 \times 10^{-4} \mathrm{~N} / \mathrm{V}$ (b), $\alpha=3.1 \times 10^{-3} \mathrm{~N} / \mathrm{V}$ (c), and $\alpha=3.1 \times 10^{-2} \mathrm{~N} / \mathrm{V}$ (d). 

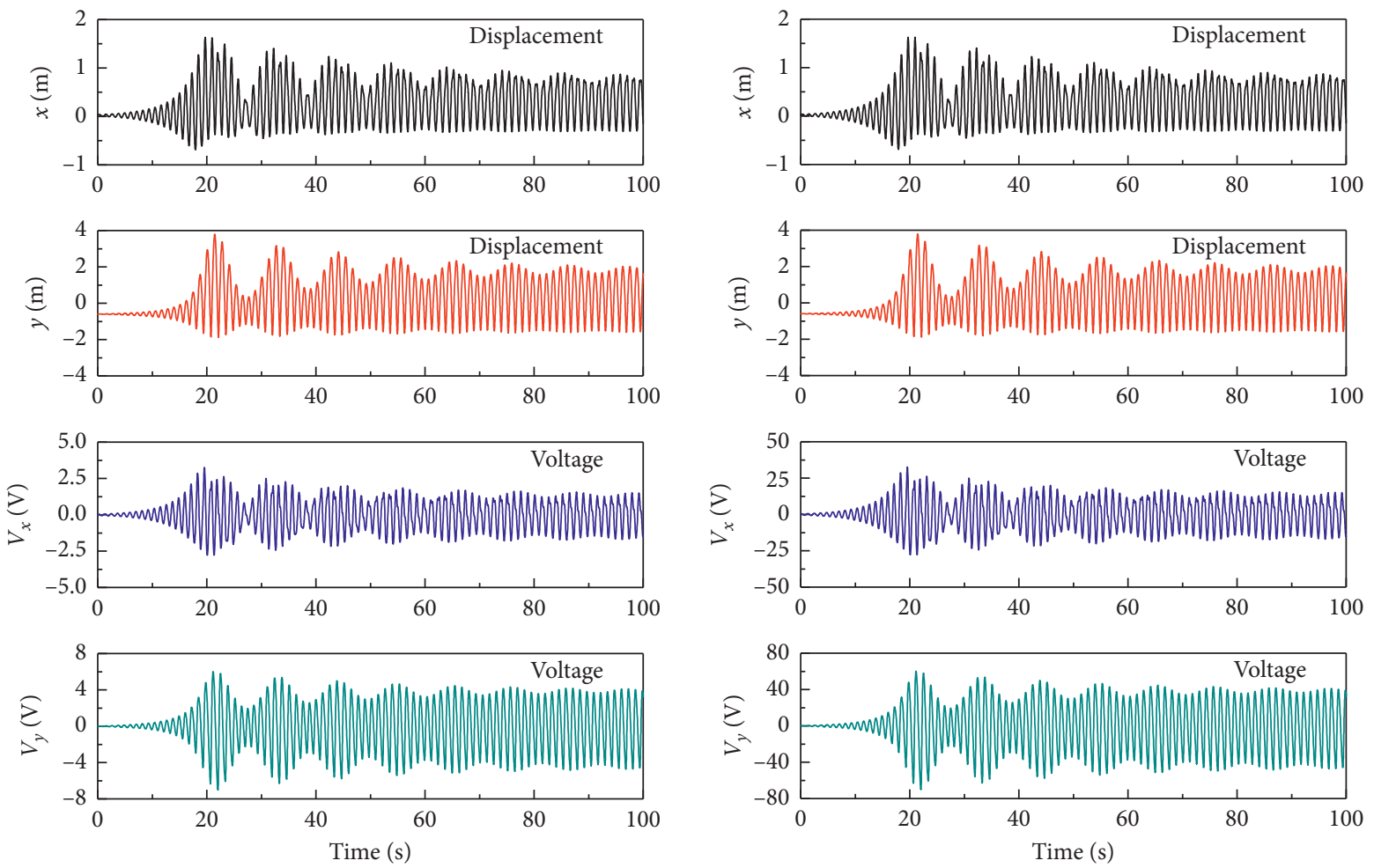

(a)

(b)
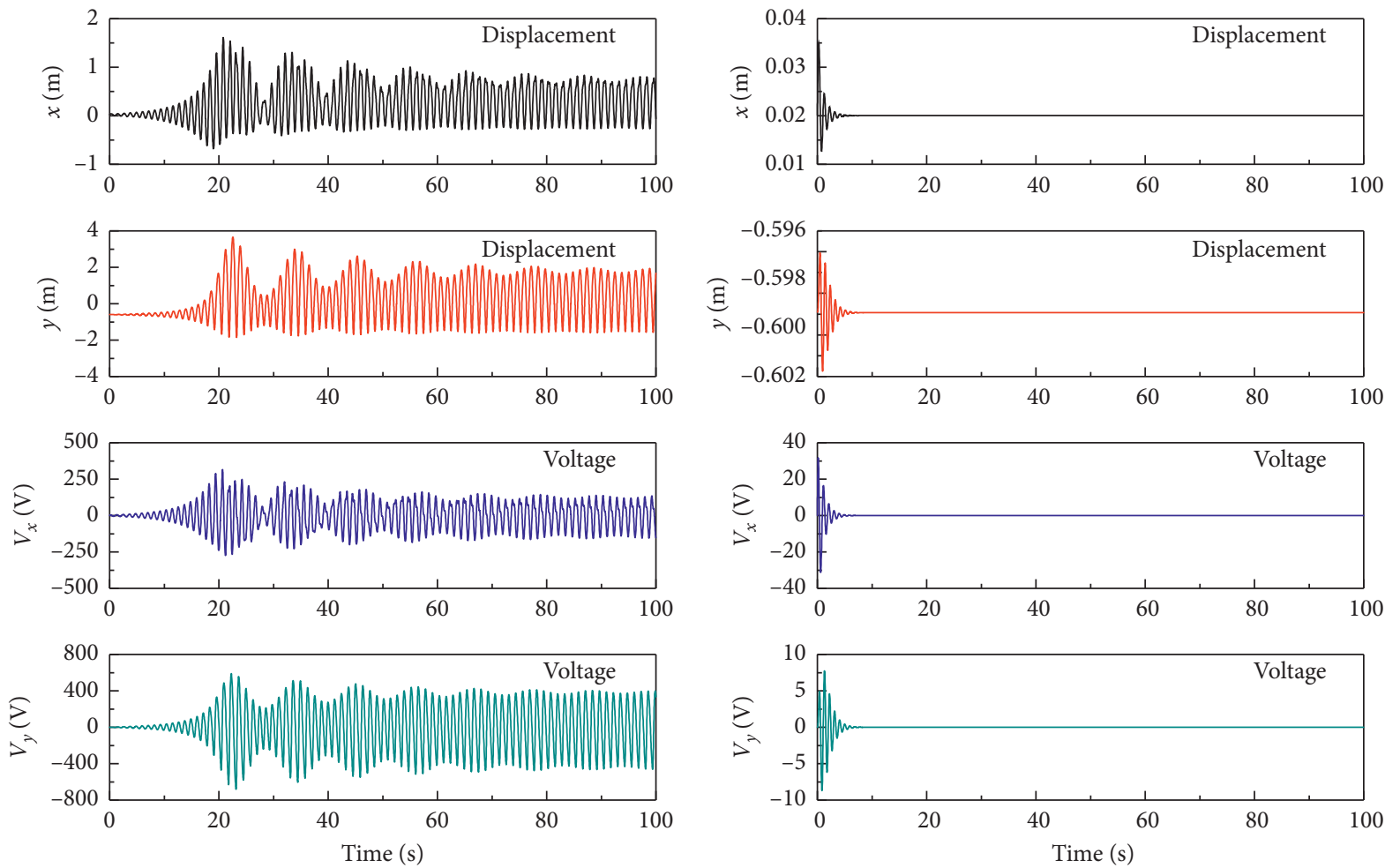

(c)

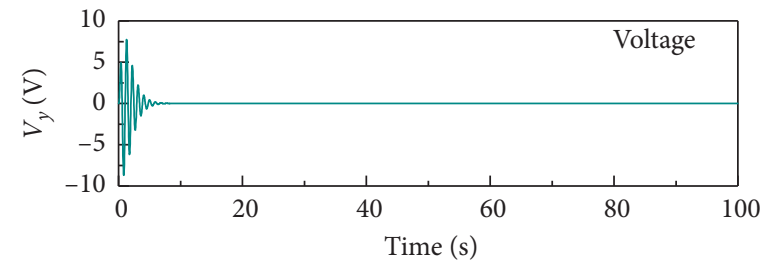

(d)

Figure 30: Considering separation, the displacements and output voltages of the friction system when the force factor of piezoelectric elements is $\alpha=3.1 \times 10^{-5} \mathrm{~N} / \mathrm{V}$ (a), $\alpha=3.1 \times 10^{-4} \mathrm{~N} / \mathrm{V}$ (b), $\alpha=3.1 \times 10^{-3} \mathrm{~N} / \mathrm{V}$ (c), and $\alpha=3.1 \times 10^{-2} \mathrm{~N} / \mathrm{V}$ (d). 


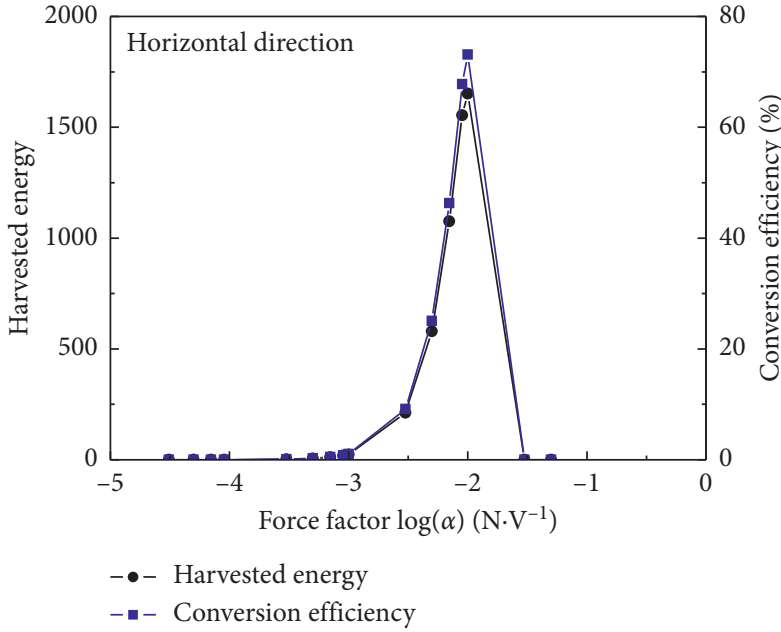

(a)

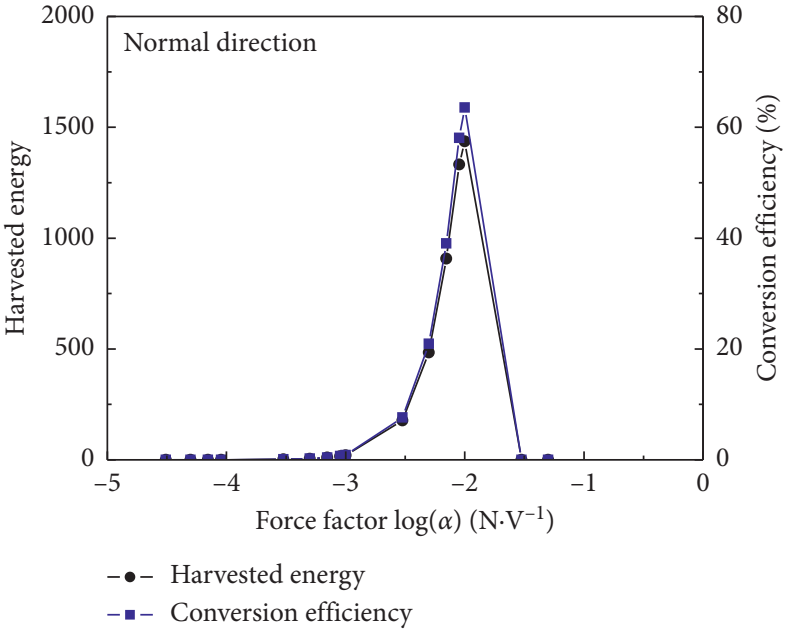

(b)

FIGURE 31: Ignoring separation, the harvested energy and conversion efficiency of the friction system in the horizontal direction (a) and normal direction (b) with different force factors of piezoelectric elements.

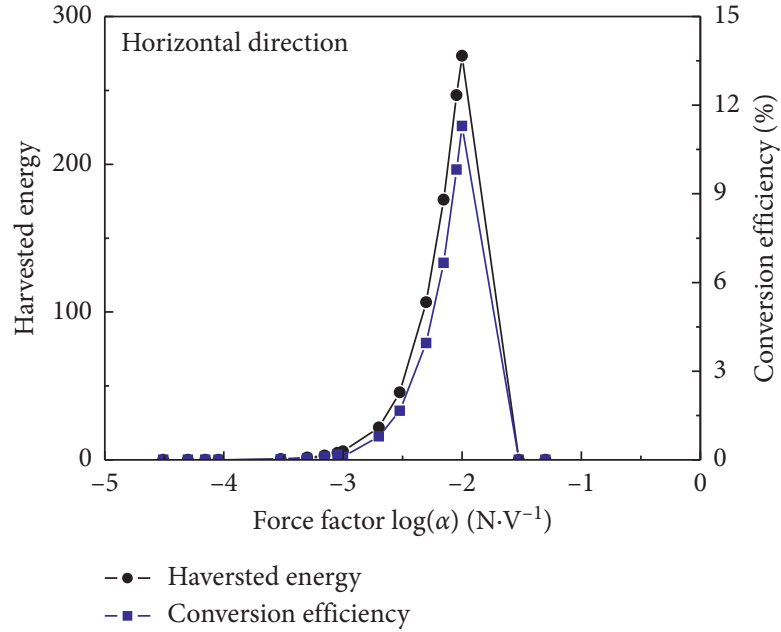

(a)

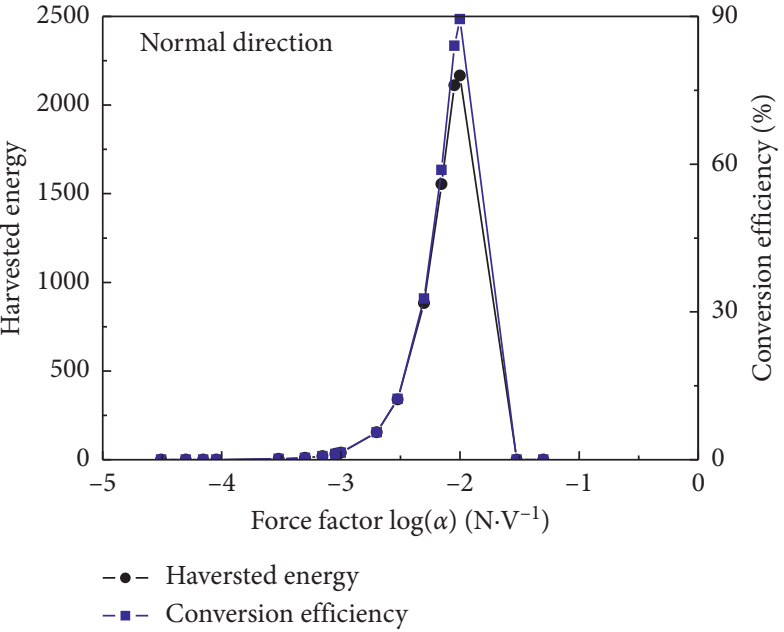

(b)

FIgURE 32: Considering separation, the harvested energy and conversion efficiency of the friction system in the horizontal direction (a) and normal direction (b) with different force factors of piezoelectric elements.

\section{Conclusions}

In this study, the piezoelectric energy harvesting behaviour by means of friction-induced vibration is studied numerically, a one-degree-of-freedom system with friction and a piezoelectric element are proposed, and then, two kinds of friction laws are, respectively, introduced into the system to study the piezoelectric energy harvesting behaviour related to friction-induced stick-slip vibration. Then, a two-degree-of-freedom friction system with piezoelectric elements which ignores and considers the separation between contact pairs is proposed, to further investigate the piezoelectric energy harvesting behaviour via friction-induced vibration. The main conclusions drawn from the results are as follows:
(1) It is feasible to harvest electrical energy by means of friction-induced vibration. The friction system can harvest electrical energy when the friction system comes into the unstable vibration region.

(2) The proposed two friction systems represent two kinds of unstable vibration, i.e., stick-slip instability and mode coupling instability. These two friction systems can be used in the stick-slip vibration energy harvesting and mode coupling vibration energy harvesting, respectively.

(3) The normal load can significantly affect the dynamic and energy harvesting behaviour of the friction system. The amount of the harvested energy can be increased by increasing the normal load or driving 
velocity. Due to the differences of friction laws used in the stick-slip motion, the conversion efficiency may remain steady or show a peak with the variation of the critical load or driving velocity.

(4) The external electric resistance can modify the output voltage amplitudes within limits; an appropriate external electric resistance value is beneficial for achieving the highest harvested energy and conversion efficiency.

(5) There exists an optimized force factor of piezoelectric element which can give the highest harvested energy and conversion efficiency.

This research provides a platform for investigating the effect of several parameters on the energy harvesting performance of theologically friction systems. Further research work planned includes design of an experimental machine and tests to validate the energy harvesting behaviour by means of friction-induced vibration. In the recent work performed by Di Bartolomeo et al. [58], they found that broadband excitation can be generated from the contact surface when the friction system arrives stable sliding phase; therefore, the stable friction vibration response should be considered in energy harvesting as well. On the other hand, the research on the variation of the energy harvesting performances when the friction system switches from the stable regime to unstable stick-slip or mode coupling regime will be investigated as well.

\section{Data Availability}

The data used to support the findings of this study are included within the article.

\section{Conflicts of Interest}

The authors declare no potential conflicts of interest with respect to the research, authorship, and/or publication of this article.

\section{Acknowledgments}

This research received the financial supports from the National Natural Science Foundation of China (no. 51505396).

\section{References}

[1] S. Bi, C. K. Ho, and R. Zhang, "Wireless powered communication: opportunities and challenges," IEEE Communications Magazine, vol. 53, no. 4, pp. 117-125, 2015.

[2] S. P. Beeby, M. J. Tudor, and N. M. White, "Energy harvesting vibration sources for micro systems applications," Measurement Science and Technology, vol. 17, no. 12, pp. R175-R195, 2006.

[3] H. S. Kim, J.-H. Kim, and J. Kim, "A review of piezoelectric energy harvesting based on vibration," International Journal of Precision Engineering and Manufacturing, vol. 12, no. 6, pp. 1129-1141, 2011.

[4] L. Tang, Y. Yang, and C. K. Soh, "Toward broadband vibration-based energy harvesting," Journal of Intelligent $\mathrm{Ma}$ terial Systems and Structures, vol. 21, no. 18, pp. 1867-1897, 2010.
[5] A. Erturk and D. Inman, Piezoelectric Energy Harvesting, John Wiley \& Sons Ltd., Chichester, UK, 2011.

[6] N. G. Stephen, "On energy harvesting from ambient vibration," Journal of Sound and Vibration, vol. 293, no. 1-2, pp. 409-425, 2006.

[7] S. Saadon and O. Sidek, "A review of vibration-based MEMS piezoelectric energy harvesters," Energy Conversion and Management, vol. 52, no. 1, pp. 500-504, 2011.

[8] J. Twiefel and H. Westermann, "Survey on broadband techniques for vibration energy harvesting," Journal of Intelligent Material Systems and Structures, vol. 24, no. 11, pp. 1291-1302, 2013.

[9] X. Wang, Frequency Analysis of Vibration Energy Harvesting Systems, Elsevier, Amsterdam, Netherlands, 2016.

[10] C. Wei and X. Jing, "A comprehensive review on vibration energy harvesting: modelling and realization," Renewable and Sustainable Energy Reviews, vol. 74, pp. 1-18, 2017.

[11] D. W. Wang, J. L. Mo, X. F. Wang, H. Ouyang, and Z. R. Zhou, "Experimental and numerical investigations of the piezoelectric energy harvesting via friction-induced vibration," Energy Conversion and Management, vol. 171, pp. 1134-1149, 2018.

[12] A. Erturk and D. J. Inman, "On mechanical modeling of cantilevered piezoelectric vibration energy harvesters," Journal of Intelligent Material Systems and Structures, vol. 19, no. 11, pp. 1311-1325, 2008.

[13] A. Erturk and D. J. Inman, "An experimentally validated bimorph cantilever model for piezoelectric energy harvesting from base excitations," Smart Materials and Structures, vol. 18, no. 2, Article ID 025009, 2009.

[14] M. N. Fakhzan and A. G. A. Muthalif, "Harvesting vibration energy using piezoelectric material: modeling, simulation and experimental verifications," Mechatronics, vol. 23, no. 1, pp. 61-66, 2013.

[15] M. Kim, J. Dugundji, and B. L. Wardle, "Efficiency of piezoelectric mechanical vibration energy harvesting," Smart Materials and Structures, vol. 24, Article ID 055006, 2015.

[16] V. R. Challa, M. G. Prasad, and F. T. Fisher, "Towards an autonomous self-tuning vibration energy harvesting device for wireless sensor network applications," Smart Materials and Structures, vol. 20, no. 2, pp. 1-11, 2011.

[17] N. E. Dutoit, B. L. Wardle, and S. G. Kim, "Design considerations for mems-scale piezoelectric mechanical vibration energy harvesters," Integrated Ferroelectrics, vol. 71, no. 1, pp. 121-160, 2005.

[18] H. W. Kim, A. Batra, S. Priya et al., "Energy harvesting using a piezoelectric "cymbal" transducer in dynamic environment," Japanese Journal of Applied Physics, vol. 43, no. 9A, pp. 6178-6183, 2004.

[19] E. S. Leland and P. K. Wright, "Resonance tuning of piezoelectric vibration energy scavenging generators using compressive axial preload," Smart Materials and Structures, vol. 15, no. 5, pp. 1413-1420, 2006.

[20] J. Q. Liu, H. B. Fang, Z. Y. Xu et al., "A MEMS-based piezoelectric power generator array for vibration energy harvesting," Microelectronics Journal, vol. 39, no. 5, pp. 802-806, 2008.

[21] H. D. Li, C. Tian, and Z. D. Deng, "Energy harvesting from low frequency applications using piezoelectric materials," Applied Physics Reviews, vol. 1, no. 4, Article ID 041301, 2014.

[22] D. Shen, J.-H. Park, J. H. Noh et al., "Micromachined PZT cantilever based on SOI structure for low frequency vibration energy harvesting," Sensors and Actuators A: Physical, vol. 154, no. 1, pp. 103-108, 2009.

[23] S. Du, Y. Jia, and A. A. Seshia, "An efficient inductorless dynamically configured interface circuit for piezoelectric 
vibration energy harvesting," IEEE Transactions on Power Electronics, vol. 32, no. 5, pp. 3595-3609, 2017.

[24] D. Guyomar, G. Sebald, S. Pruvost, M. Lallart, A. Khodayari, and C. Richard, "Energy harvesting from ambient vibrations and heat," Journal of Intelligent Material Systems and Structures, vol. 20, no. 5, pp. 609-624, 2009.

[25] E. Lefeuvre, A. Badel, C. Richard, L. Petit, and D. Guyomar, "A comparison between several vibration-powered piezoelectric generators for standalone systems," Sensors and Actuators A: Physical, vol. 126, no. 2, pp. 405-416, 2006.

[26] L. Tang and Y. Yang, "A multiple-degree-of-freedom piezoelectric energy harvesting model," Journal of Intelligent Material Systems and Structures, vol. 23, no. 14, pp. 1631-1647, 2012.

[27] X. Wang and L. W. Lin, "Dimensionless optimization of piezoelectric vibration energy harvesters with different interface circuits," Smart Materials and Structures, vol. 22, no. 8, Article ID 085011, 2013.

[28] F. Cottone, H. Vocca, and L. Gammaitoni, "Nonlinear energy harvesting," Physical Review Letters, vol. 102, no. 8, pp. 1-4, 2009.

[29] L. Gammaitoni, I. Neri, and H. Vocca, "Nonlinear oscillators for vibration energy harvesting," Applied Physics Letters, vol. 94, no. 16, Article ID 164102, 2009.

[30] B. P. Mann and B. A. Owens, "Investigations of a nonlinear energy harvester with a bistable potential well," Journal of Sound and Vibration, vol. 329, no. 9, pp. 1215-1226, 2010.

[31] B. P. Mann and N. D. Sims, "Energy harvesting from the nonlinear oscillations of magnetic levitation," Journal of Sound and Vibration, vol. 319, no. 1-2, pp. 515-530, 2009.

[32] S. C. Stanton, C. C. McGehee, and B. P. Mann, "Nonlinear dynamics for broadband energy harvesting: investigation of a bistable piezoelectric inertial generator," Physica D: Nonlinear Phenomena, vol. 239, no. 10, pp. 640-653, 2010.

[33] L. Simeone, M. G. Tehrani, S. J. Elliott et al., "Nonlinear damping in an energy harvesting device," in Proceedings of the ISMA 2014 International Conference on Noise and Vibration Engineering, Leuven, Belgium, September 2014.

[34] W. J. Ding, Self-Excited Vibration, Springer, Amsterdam, Netherlands, 2010.

[35] D. Li, Y. Wu, A. Da Ronch, and J. Xiang, "Energy harvesting by means of flow-induced vibrations on aerospace vehicles," Progress in Aerospace Sciences, vol. 86, pp. 28-62, 2016.

[36] A. Abdelkefi, "Aeroelastic energy harvesting: a review," International Journal of Engineering Science, vol. 100, pp. 112135, 2016.

[37] R. A. Ibrahim, "Friction-induced vibration, chatter, squeal, and chaos-part II: dynamics and modeling," Applied Mechanics Reviews, vol. 47, no. 7, pp. 227-253, 1994.

[38] J. Kang and K. Kim, "Squeak noise in lead screw systems: selfexcited vibration of continuous model," Journal of Sound and Vibration, vol. 329, no. 17, pp. 3587-3595, 2010.

[39] J. Le Rouzic, A. Le Bot, J. Perret-Liaudet et al., "Frictioninduced vibration by Stribeck's law: application to wiper blade squeal noise," Tribology Letters, vol. 49, no. 3, pp. 563-572, 2013.

[40] G. X. Chen and Z. R. Zhou, "A self-excited vibration model based on special elastic vibration modes of friction systems and time delays between the normal and friction forces: a new mechanism for squealing noise," Wear, vol. 262, no. 9-10, pp. 1123-1139, 2007.

[41] M. R. North, "Disc brake squeal," Proceedings of the Institution of Mechanical Engineers, vol. C38/76, pp. 169-176, 1976.
[42] H. Ouyang, "Moving-load dynamic problems: a tutorial (with a brief overview)," Mechanical Systems and Signal Processing, vol. 25, no. 6, pp. 2039-2060, 2011.

[43] S. K. Rhee, P. H. S. Tsang, and Y. S. Wang, "Friction-induced noise and vibration of disc brakes," Wear, vol. 133, no. 1, pp. 39-45, 1989.

[44] R. T. Spurr, "A theory of brake squeal," Proceedings of the Institution of Mechanical Engineers: Automobile Division, vol. 15, no. 1, pp. 33-52, 1961.

[45] L. Hammerström and S. Jacobson, "Surface modification of brake discs to reduce squeal problems," Wear, vol. 261, no. 1, pp. 53-57, 2006.

[46] F. Massi and O. Giannini, "slightEffect of damping on the propensity of squeal instability: an experimental investigation," The Journal of the Acoustical Society of America, vol. 123, no. 4, pp. 2017-2023, 2008.

[47] C. L. Saw, C. G. Choong, A. R. AbuBakar et al., "Disc brake squeal suppression through chamfered and slotted pad," International Journal of Vehicle Structures \& Systems, vol. 3, no. 1, pp. 28-35, 2011.

[48] M. Triches Jr., S. N. Y. Gerges, and R. Jordan, "Reduction of squeal noise from disc brake systems using constrained layer damping," Journal of the Brazilian Society of Mechanical Sciences and Engineering, vol. 26, no. 3, pp. 340-348, 2004.

[49] D. W. Wang, J. L. Mo, H. Ouyang, G. X. Chen, M. H. Zhu, and Z. R. Zhou, "Experimental and numerical studies of frictioninduced vibration and noise and the effects of groove-textured surfaces," Mechanical Systems and Signal Processing, vol. 46, no. 2, pp. 191-208, 2014.

[50] N. M. Ghazaly, M. El-Sharkawy, and I. Ahmed, "A review of automotive brake squeal mechanisms," Journal of Mechanical Design and Vibration, vol. 1, no. 1, pp. 5-9, 2014.

[51] L. E. Helseth, "Excitation of energy harvesters using stick-slip motion," Smart Materials and Structures, vol. 23, no. 8, Article ID 085024, 2014.

[52] C. Tadokoro, A. Matsumoto, T. Nagamine et al., "Piezoelectric power generation using friction-induced vibration," Smart Materials and Structures, vol. 26, no. 6, Article ID 065012, 2017.

[53] A. Masuda and C. Sawai, "Stick-slip energy harvesting: a preliminary study," in Proceedings of the ASME 2017 Conference on Smart Materials, Adaptive Structures and Intelligent Systems, Snowbird, UT, USA, September 2017.

[54] E. Heffel and P. Hagedorn, "Friction induce vibration for energy harvesting applications," in Proceedings of the 10th International Workshop on Piezoelectric Materials and Applications and 8th Energy Harvesting Workshop (IWPMA/ EHW 2013), Hannover, Germany, July 2013.

[55] D. Tonazzi, F. Massi, L. Baillet, A. Culla, M. Di Bartolomeo, and Y. Berthier, "Experimental and numerical analysis of frictional contact scenarios: from macro stick-slip to continuous sliding," Meccanica, vol. 50, no. 3, pp. 649-664, 2015.

[56] Z. L. Li, H. Ouyang, and Z. Q. Guan, "Nonlinear frictioninduced vibration of a slider-belt system," ASME Journal of Vibration and Acoustics, vol. 138, no. 4, Article ID 041006-9, 2016.

[57] A. Medio and M. Lines, Nonlinear Dynamics: A Primer, Cambridge University Press, Cambridge, UK, 2001.

[58] M. Di Bartolomeo, G. Lacerra, L. Baillet, E. Chatelet, and F. Massi, "Parametrical experimental and numerical analysis on friction-induced vibrations by a simple frictional system," Tribology International, vol. 112, pp. 47-57, 2017. 